Nationai Library

of Canada

Acquisitions and

Bioliographic Services Branch

395 Wellington Sticel

Otiawa. Ontario

KIA ON4
Bibliotheque nationale

du Canada

Direction des acquisitions el

des services bibliographiques

395. rue Wellington

Otlawa (Ontario)

KIA ONA

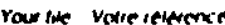

Qun hite Notieteterence

NOTICE

AVIS heavily dependent upon the quality of the original thesis submitted for microfilming. Every effort has been made to ensure the highest quality of reproduction possible.

If pages are missing, contact the university which granted the degree.

Some pages may have indistinct print especially if the original pages were typed with a poor typewriter ribbon or if the university sent us an inferior photocopy.
The quality of this microform is

La qualité de cette microforme dépend grandement de la qualité de la thèse soumise au microfilmage. Nous avons tout fait pour assurer une qualité supérieure de reproduction.

S'il manque des pages, veuillez communiquer avec l'université qui a conféré le grade.

La qualité d'impression de certaines pages peut laisser à désirer, surtout si les pages originales ont été dactylographiées à l'aide d'un ruban usé ou si l'université nous a fait parvenir une photocopie de qualité inférieure.

Reproduction in tult or in part of this microform is governed by the Canadian Copyright Act, R.S.C. 1970, c. C-30, and subsequent amendments.
La reproduction, même partielle, de cette microforme est soumise à la Loi canadienne sur le droit d'auteur, SRC 1970, c. C-30, et ses amendements subséquents. 


\title{
Quantum Hall Effect in the Presence of an Antidot Potential
}

\author{
Ming Lei
}

Centre for the Physics of Materials

Department of Physics, McGill University

Montréal, Québec

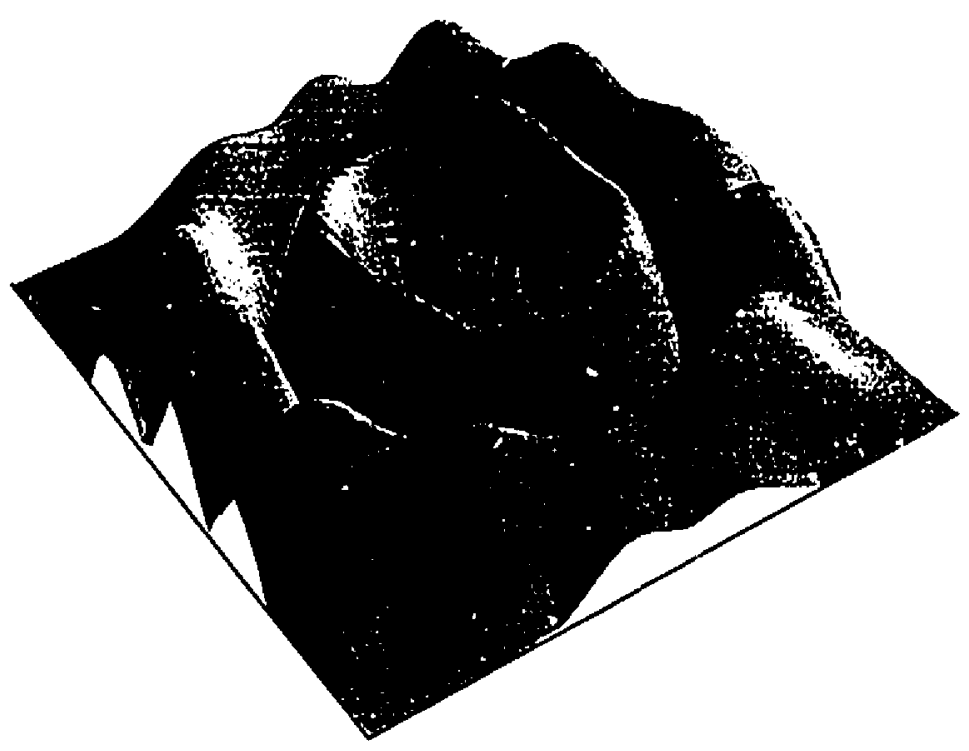

A Thesis submitted to the

Faculty of Graduate Studies and Research

in partial fulfillment of the requirements for the degree of Master of Science

(C) Ming Lei 1996 
National Library

of Canada

Acquisitions and

Bibliographic Services Branch

395 Wellington Street

Ottawa Ontario

KIA ON4
Bibliothèque nationale

du Canada

Direction des acquisitions et des services bibliographiques

395, rue Wellington

Ottawa (Ontario)

K1AONA
Your like vole reterence

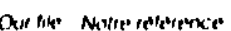

The author has granted an irrevocable non-exclusive licence allowing the National Library of Canada to reproduce, loan, distribute or sell copies of his/her thesis by any means and in any form or format, making this thesis available to interested persons.

The author retains ownership of the copyright in his/her thesis. Neither the thesis nor substantial extracts from it may be printed or otherwise reproduced without his/her permission.
L'auteur a accordé une licence irrévocable et non exclusive permettant à la Bibliothèque nationale du Canada de reproduire, prêter, distribuer ou vendre des copies de sa thèse de quelque manière et sous quelque forme que ce soit pour mettre des exemplaires de cette thèse à la disposition des personnes intéressées.

L'auteur conserve la propriété du droit d'auteur qui protège sa thèse. Ni la thèse ni des extraits substantiels de celle-ci ne doivent être imprimés ou autrement reproduits sans son autorisation. 
to my parents and my sister. 


\section{CONTENTS}

Abstract viii

RÉSUMÉ ix

ACKNOWLEDGMENTS $\mathbf{x}$

$\mathbf{1}$ INTRODUCTION

1.1 Outline ...................... 4

2 Quantum Hall Effect and Aharonov-Bohm EFfect 6

2.1 Introduction . . . . . . . . . . . . . . 6

2.2 Basic Concepts of Hall Effect $\ldots \ldots \ldots \ldots \ldots \ldots \ldots$

2.3 Discovery of Quantum Hall Effect . . . . . . . . . . . . 10

2.4 Theoretical Explanations of Quantum Hall Effect . . . . . . . . 12

2.4.1 Laughlin's Explanation . . . . . . . . . . . . . 13

2.4 .2 Halperin's Explanation . . . . . . . . . . . . 16

2.5 Aharonov-Bohm Effect . . . . . . . . . . . . . 20

2.6 Relation of Two Effects . . . . . . . . . . . . . . . . 22

3 BÜtTIKeR's Theory OF Electrical Conduction 25

3.1 Introductory Remarks . . . . . . . . . . . . . 25

3.2 The Scattering Problem $\ldots \ldots \ldots \ldots \ldots \ldots \ldots \ldots$

3.3 The Current Response due to Differing Chemical Potentials . . . . 30

3.4 Resistance and Transmission . . . . . . . . . . . . 33

4 Effect of an Antidot Potential 36

4.1 Introduction . . . . . . . . . . . . . . 36

4.2 Conductor with Ideal Propagation Channels . . . . . . . . . 37

4.3 Behavior of the Hall Resistance . . . . . . . . . . . . . 39

4.4 Crossover Regime . . . . . . . . . . . . . . . . . . 49

4.5 Topological Explanation of Crossover Regime . . . . . . . . 54

4.6 Effect of Resonant State on the Quantum Hall Effect . . . . . . . 60

4.7 Universality of Micro-symmetry in Crossover Regime . . . . . . 66

5 ConClusions $\quad \mathbf{7 9}$

APPENDIX $\quad 81$

A.1 Definitions of Different Transmission Probabilities . . . . . . 81

$\begin{array}{ll}\text { REFERENCES } & \mathbf{8 2}\end{array}$ 


\section{Figures and Tables}

\section{FIGURES}

2.1 Density of staies of 2DEG and 3DEG in magnetic field. . . . . . . . 9

2.2 Quantum Hall effect observed on the inversion layer of Si-MOSFET. . 11

2.3 Density of states without (top) and with (bottom) disorder. . . . . 12

2.4 Left: Diagram of metallic loop. Right: Corbino plate. . . . . . . . 14

2.5 Schematic plot of geometry of sample of Halperin's explanation of quantum Hall effect. . . . . . . . . . . . . . . . 16

2.6 Quantum channels in high magnetic field for a perfect conductor. . . 18

2.7 Schematic illustration of the geometry which results in periodic AharonovBchm oscillations. ..................... 20

2.8 A schematic representation of transport through an annulus in the $A B$ effect regime (a), and in the quantum Hall regime (b). . . . . . . 23

3.1 Schematic plot of a conductor with a number of probes permitting carriers to enter and leave. . . . . . . . . . . . . . .

3.2 Schematic plot of a conductor with probes connected to electron reser-

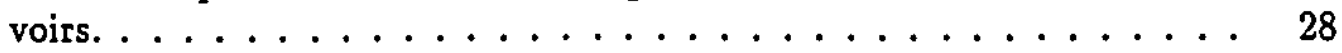

4.1 Schematic view of a conductor with Hall probes and perfect edge states. 38

4.2 Schematic view of the Hall junction with an antidot confined in it. . . 40

4.3 Hall resistance $R_{H}$ as a function of magnetic field $B$ for different antidot sizes $r_{a} \ldots \ldots \ldots \ldots \ldots \ldots \ldots$

4.4 The norm $|\Psi(x, y)|^{2}$ for $r_{a}=W / 4$ in the quanturn Hall regime. . . . 46

4.5 The Contour of norm of wave function for $r_{a}=3 W / 4$ at two different

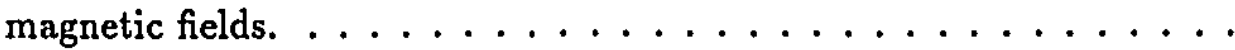

4.6 (a): transmission coefficients $T_{m n}$ of square-shaped Hall junction versus antidot size $r_{a}$. (b). Hall resistance $R_{H}$ of square-shaped Hall junction versus antidot size $r_{a} \ldots \ldots \ldots \ldots \ldots \ldots$

4.7 (a) Transmission coefficients of individual incoming channels $T_{m n}^{i}$ of square-shaped Hall junction versus antidot size $r_{a}$ at $B=5450$ Gauss.

(b) Transmission coefficients of individual incoming channels $T_{m n}^{i}$ of square-shaped Hall junction as a function of magnetic field $B$ at $r_{a}=$

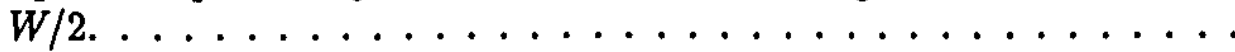

4.8 Schematic plot of the two important transmission patterns in the Hall

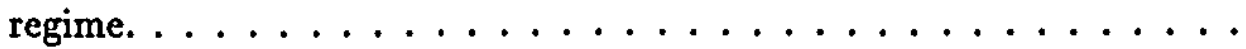

4.9 Schematic plot of the extrapolation parameters $\alpha_{i}\left(r_{a}, B\right)$ and $\beta_{i}\left(r_{a}, B\right)$ as extracted from the numerical data. . . . . . . . . . . . 59

$4.10 \mathrm{Hall}$ resistance $R_{H}$ of two transmission patterns with one edge state only. 62

4.11 Hall resistance $R_{H}$ versus magnetic field $B$ for small antidot size with incoming electron in channel 1 and 2 respectively. . . . . . . . 
4.12 Hall resistance $R_{H}$ versus magnetic field $B$ for intermediate antidot size with incoming electron in channel 1 and 2 respectively. . . . . . 65

4.13 Schematic view of the circular-shaped Hall junction. . . . . . . . 67

4.14 (a): Transmission coefficients $T_{m n}$ of circular-shaped Hall junction versus antidot size $r_{a}$. (b). Hall resistance $R_{H}$ of circular-shaped Hall junction versus antidot size $r_{\mathrm{a}} \ldots \ldots \ldots 68$

4.15 Transmission coefficients of circular-shaped Hall junction of individual incoming channels $T_{m n}^{i} \ldots \ldots \ldots \ldots \ldots \ldots$

4.16 Schematic view of Hall junctions with an antidot not locating at the center of the structures. ................. 70

4.17 Transmission coefficients of the structure shown in Fig. (4.16a). . . . 72

4.18 Transmission coefficients of the structure shown in Fig. (4.16b). . . . 73

4.19 Transmission coefficients of the structure shown in Fig. (4.16c). . . . 74

4.20 Transmission coefficients of the structure shown in Fig. (4.16d). . . . 75

4.21 Hall resistance $R_{H}$ as a function of the antidot size $r_{a}$ at $B=6060$ Gauss for Hall junctions shown in Fig. (4.16). . . . . . . . . . . 78

\section{TABLES}

4.1 Transmission coefficients $T_{i j, m n}$ as a function of magnetic ficld at $r_{a}=0.6 \mathrm{~W} .52$

4.2 Transmission coefficients $T_{i j_{1} m n}$ for different antidot size $r_{a}$ at $B=6172$

Gauss. ........................ 53 


\section{ABSTRACT}

The crossover transport regime between the quantum Hall effect and the AharonovBohm effect is studied in terms of Büttiker's approach of electrical conduction. Quantum Hall effect and Aharcnov-Bohm effect are very important effects in mesoscopic physics and both demonstrate unambiguously that quantum mechanics is the dominant factor in nanoscale electrical transport problems. However, they belong to situations of different dimensionality and different strength of magnetic fields. Our goal is to reveal the physics at the crossover regime between the two and find the transport properties of this transition regime.

We have computed Hall resistance of a four-probe box-shaped quantum dot with an artificial impurity confined inside. As the size of the impurity is increased, transport behavior changes from the usual quantum Hall regime to a regime dominated by strong Aharonov-Bohm ( $A B)$ oscillations. We observe directly the formation and coupling of the edge states and their effects on the Hall resistance, by varying a magnetic field. For a range of the impurity size, transport enters the crossover regime where quantum Hall and $A B$ effects compete, and a peculiar approximate symmetry between various transmission coefficients lead to a Hall plateau before the quantum Hall regime is reached. This symmetry can be explained based on scattering matrix theory and a topological equivalence of the dominating transmission patterns where well defined edge states are formed. Finally we investigate the universality of the observed symmetry property in several other structures and find that within the scope of our calculation the symmetry is universal. 


\section{RÉSUMÉ}

Le régime intermédiaire de transport entre l'effet Hall quantique et l'effet d'AlaronovBohm est étudié en utilisant la conduction électrique de Büttiker. L'effet Hall quantique et l'effet d'Aharonov-Bohm sont des effets très importants en physique mésoscopique et ils démontrent tous les deux clairement que la mécanique quantique est le facteur dominant dans les problèmes de transport électrique à l'échelle du nanomètre. Toutefois, ils appartiennent à des situations d'autres dimensions et à différentes forces du champ magnétique. Notre objectif est d'étudier la physique reliée au régime intermédiaire entre les deux et de trouver les propriétés de ce régime cle transition.

Nous avons calculé la résistance de Hall d'un point quantique cubique muni de quatre sondes et d'une impureté artificielle logée à l'intérieur dı point. En augmentant la dimension de l'impureté, le transport passe du régime de Hall, que nous retrouvons habituellement, à un régime qui est doniné par de fortes oscillations d'AharonovBohm (AB). Nous observons directement la formation et le couplage des états limites et leur influence sur la résistance de Hall, en variant le champ magnétique. Pour une gamme de dimension d'impuretés, le transport passe au régime intermédiaire où l'effet Hall quantique et l'effet $A B$ sont en compétition. Une symétrie approximative particuliaire entre plusieurs coefficients de transmission mène à un plateau de Hall avant que le régime de Hall quantique soit atteint. Cette symétrie peut être expliquée en se basant sur la théorie de la matrice de rétrodiffusion et sur l'équivalence topologique des patrons de transmission dominants où des états limites bien définis sont formés. Finalement, nous avons étudié l'universalité de la propriété de symétrie dans plusieurs autres structures et nous avons trouvé que la symétrie est universelle à l'intérieur de l'étendue de nos calculs. 


\section{ACKNOWLEDGMENTS}

It would have been impossible for me to complete this thesis without the presence and support of many people. First I would like to thank Prof. Hong Guo, my supervisor, for introducing me to a very exciting field of research, for his constant intellectual stimulation, and for his support throughout my stay at McGill. This thesis benefitted from his careful reading and constructive criticism. For this I am very grateful.

I wish to acknowledge the close collaboration of my friend and fellow student Ningiia Zhu on mesoscopic physics. With Ningiia's help, my working experience has improved, and his constructive criticisms were always appreciated. I am grateful to my dear friend Dr. Brett Ellman for helping me solve the problems of editing the thesis, often done at very short notice! I would like to thank Dr. Yongjiang Wang for his generous gift - an excellent calculation program, of course a very complicated one which prevented me from sleeping for a whole menth! Special thanks goes to Christin for rendering the French version of the abstract.

A special thanks is addressed especially to my teachers at McGill. I benefitted from the courses I took from Profs. Martin Grant, Hong Guo, Mark Sutton and Robert Myers.

I have enjoy the intellectual stimulation and the friendship of many people at McGill. Thanks to my colleagues, Ningjia, Niri, Haiqing, Gianni and Mozos for many very stimulating discussions from which I always learned something. I also enjoyed discussions and good times with my office mates and friends of room 420 (ladies first): Randa, Rita, Mary, Ying, Ou M., Maxim, Zhida, Yi Zhao, Yanzhang and Christian... During my stay at McGill, I had the pleasure to meet wonderful people who made my stay in Montreal an experience to remember: Biao, Brett, Christian, Christin, Erol, Francois, Geoff, Haiqing, Jun, Karin, Ling, Lu, Martin L., Mikko, Mohsen, Mozos, Ningjia, Ou M., Sajan, Yanzhang, little Ren, old Xiao, Hong, Martin G., Martin Z. ... I will miss you all!

I would like to thank McGill University for providing me financial support through a Max Stern Fellowship over the past two years. Also, thanks to Prof. Hong Guo for additional support. Many thanks also to Paula Domingues, Diane Koziol, Joanne Longo, Cynthia Thomas and Lynda Corkum for making dealing with the department both simple and always pleasant.

Finally, I wish to express my gratitude to my parents and my sister for their 
support which has been invaluble to me so far and will be for many more years to come. 


\section{Quantum Hall Effect in the Presence of an Antidot Potential}




\section{1}

\section{INTRODUCTION}

Due to the advancement of nanofabrication techniques, in the last several years a host of very interesting low dimensional electronic devices have been produced which present opportunities for understanding quantum transport in nanoscale systems[1]. Well known examples of these systems include the quantum point contact[2, 3], quantum wires or electron waveguides[4], quantum dots or artificial atoms $[4,5]$, and combinations of these. The sizes of these systems can be so small such that charge carriers traverse them without on average feeling any impurity scattering. At the very low temperatures where most experiments were carried out, one may also neglect such inelastic effects as electron-phonon scattering. In this regime of transport, the current limiting factor is largely provided by the boundary of the device and the transport is phase coherent throughout[4]. Today, we have reached at a level of solid-statc structural sophistication where we commonly design and fabricate devices based primarily on quantum mechanical principles. This "designer capability" has opened up a fascinating new realm of fundamental physics to be explored.

Semiconductor material of high purity and crystalline perfection can be structured to contain a thin layer of highly mobile electrons. Motion perpendicular to this layer is quantized, so that electrons are constrained to move in a plane. This two dimensional electron gas (2DEG) combines a number of desirable properties not shared by thin metal films. It has a low electron density, which may be readily varied by means of an electric field. The low density implies a large Fermi wavelength, typically $40 \mathrm{~nm}$, comparable to typical dimensions of the smallest structures (nanostructures) that can be fabricated today. The electron mean free path can be quite large, usually exceeding $10 \mu m$. The interface where the 2DEG is confined can be controlled to be atomically flat, reducing the interfacial roughness scattering of the charge carrier. The quantum 
mechanical phase coherence characteristic of a microscopic object can be maintained at low temperature (below $1 K$ ) over a distance of the order of a micron. Finally, the reduced dimensionality of the motion and the circular Fermi surface form simplifying factors for theoretical analysis. These are ideal features for applications as well as investigations of quantum transport.

Semiconductor nanostructures are unique in offering the possibility of studying quantum transport in an artificial potential landscape. This is the regime of ballistic transport, in which scattering with impurities can be neglected and one observes the effect of quantum fluctuations. Indeed, various experiments conducted on such devices observed novel phenomena directly determined by quantum interference, such as Aharonov-Bohm (AB) effect [6], quantum Hall effect ${ }^{1}[7,8]$, etc. The transport properties can then be tailored by varying the geometry of the conductor, in much the same way as one would tailor the transmission properties of a waveguide. The physics of this transport regime could be called electron optics in the solid state [9]. The formal relation between conduction and transmission, known as the Landauer formula $[10,11,12]$, has demonstrated its real power in this context. For example, the quantization of the conductance of a quantum point contact $[2,3]$ (a short and narrow constriction in the 2DEG) can be understood using the Landauer formula as resulting from the discreteness of the number of propagating modes in the construction.

The discovery of the quantum Hall effect by von Klitzing, Dorda, and Pepper is one of the most important recent discoveries in condensed matter physics and has led the research of low dimensional electronic system into a brand-new era. The quantum Hall effect provides an unparalleled opportunity to study and utilize the physical properties of macroscopic, 2DEG systems in the presence of strong magnetic fields. These studies are revealing surprising results that are of particular interest to the disciplines of condensed matter physics and electrical metrology. The Hall resistance at these quantized values is given by the fundamental unit of resistance $h / e^{2}=25812.8 \mathrm{ohm}$ divided by an integer. The development preceding this discovery is reviewed by Ando et al. [13]. The accuracy of the quantization is so high that the quantum Hall effect recently was adopted as a resistance standard. The general nature

\footnotetext{
'In this thesis, quantum Hall effect means integer quantum Hall effect.
} 
of this effect has given rise to the belief that it must have a fundamental explanation that is independent of the geometrical shape of the conductor and microscope details.

A particularly interesting nanostructure which has been used in a number of experiments is the antidot[14]. An antidot is essentially a reflecting potential peak inside an otherwise "flat" potential landscape of a two-dimensional electron gas (2DEG). In this sense an antidot could be viewed as an "artificial impurity". Single as well as multiple antidots can be fabricated by etching holes into the 2DEG[15, 16]. Anomalous quantum transport features such as chaotic scattering of the electrons[17] were observed in these systems. When the antidots form a periodic array, experiments found $[15,16]$ that some of the magneto-transport features could be correlated with the behavior of classical orbits. A simple physical picture showed $[15,16]$ that certain predominant magneto-resistance peaks were from commensurate classical orbits impaled upon a small number of nearest-neighbor antidots in the array. On the other hand, when one antidot is confined inside a quantum wire[18], quantization of the classical orbits at reasonably large magnetic field $B$ leads to the formation of magnetic edge states[19]. The important role played by edge states was clearly shown when the quantum Hall effect was explained[20,21, 22] from the point of view of these states on the basis of the Landauer theory of one-dimensional transport[11]. Essentially, the quantum Hall regime is reached when perfectly transmitting channels are formed along the edges of the sample, and according to Landauer theory, $N$ such channels or edge states contribute $N$ factors of $e^{2} / h$ to the Hall conductance, i.e. $G=N \frac{e^{2}}{h}$ where $e$ is the electron charge and $h$ the Planck constant. Thus the Hall resistance is step-like:

$$
R_{H}=\frac{h}{e^{2}} \frac{1}{N}
$$

Similar to the case of the array of antidots, here some edge states do not carry clectric current, i.e. they are localized, such as those "circulating around" the antidot. Others carry current, such as those "skipping" along the quantum wire boundaries. It was shown theoretically that coupling of the magnetic edge states leads to resonances in the quantum Hall resistances[21,23], and many phenomena in ballistic transport could be understood using this physical picture. In a more complex situation such as a 4probe Hall junction, it has been well established[24] that edge state coupling gives rise to anomalous conductance fluctuations even without an antidot. In this case $[24,25]$ 
the localized edge states presumably are formed around impurities. Similarly, the coupling of the extended and localized edge states gives rise to resonances in the magneto-conductance.

There are many important discoveries in nanostructures. Several useful reviews are listed in Refs. $[1,2,4,5,8,26]$.

\subsection{Outline}

Our work in this thesis aims to study the electronic properties when the perfect quantum Hall regime is not reached. It is well known that quantum Hall effect is operated on a 2DEG system whereas Aharonov-Bohm effect generally takes place on a ring geometry, i.e., a one dimensional sample. We will discuss these two effects and the relation between them more explicitly later on. It is the physics between them which has motivated this work. By doping an antidot in the middle of the Hall junction and varying the size of it, we can clearly demonstrate the crossover regime between the two limiting cases (quantum Hall effect and Aharonov-Bohm effect).

The contents of this thesis are as follow. Chapter 2 gives a review of quantum Hall effect and $A B$ effect. We shall recall some basic concepts of Hall effect. The discovery of quantum Hall effect and its theoretical explanations (Laughlin's and Halperin's) shall be given, and then Aharonov-Bohm effect will be discussed. In the last part of Chapter 2, we shall inspect the relation between these two effects.

In Chapter 3, Büttiker's approach [22, 27] to electrical conduction is introduced in detail. This theory is the basic tool which we use in this thesis.

In Chapter 4, a study of quantum Hall effect with the presence of an antidot potential for a box-shaped Hall-bar structure is made. A finite element scheme [28] to solve the single particle Schrödinger equation is extended to the case including a magnetic field [29]. By considering the cases without an antidot and with a very big antidot, the two limiting cases (quantum Hall effect and AB effect) are revealed clearly in our calculations. However the most interesting phenomenon is the "symmetry property" when the size of the antidot is in the middle regime [30]. A topological explanation is proposed which is based on Büttiker's topological equivalence argument [24] and $S$-matrix theory [31]. Last part of Chapter 4 is devoted to the investigation of the 
universality of the "symmetry property" observed. We study this problem from two angles. First, do we still have that symmetry when the geometric shape of the Hall junction is changed? Second, what will happen if the antidot is not located at the center of the structure? Several cases are studied and the conclusion is positive. The main results of this chapter have recently been published in Physical Review B [30]. Finally a short summary and outlook on further research is given in Chapter 5 . 


\section{2}

\section{Quantum Hall Effect and Aharonov-Bohm Effect}

\subsection{Introduction}

The quantum Hall effect was discovered at about the hundredth anniversary of Hall's original work, and the finding was announced in 1980 by von Klitzing, Dorda and Pepper [7]. Klaus von Klitzing was awarded the 1985 Nobel prize in physics for this discovery. In brief, it is found that under suitable conditions, the Hall conductivity of an inversion layer is quantized $t, i$ better than one part in $10^{5}$ to integral multiples of $e^{2} / h$. Since the discovery of quantum Hall effect, it has spurned a considerable effort to understand this phenomenon [32]. An elegant explanation of quantum Hall effect was put forth by Laughlin [33]. Laughlin discusses the response of a cylinder to an Aharonov-Bohm flux along the axis of the cylinder and explains the quantum Hall effect in terms of a supercurrent due to the long-range phase rigidity of the wave functions around the loop. Halperin [19] supplemented this picture by discussing edge states which form at the boundary of the sample. The long-range phase rigidity has led Imry [34] to propose several flux-sensitive effects. Such purely topological considerations have attracted considerable attention $[35,36,26]$.

In mesoscopic physics, there is another important effect, Aharonov-Bohm effect [6], which illustrates that in a field-free multiply-connected region of space, the physical properties of the system depend on the vector potential $\boldsymbol{A}$. Aharonov-Bohm effect actually is about quantum interference effect of a one-dimensional electronic system in the presence of a magnetic field. On the contrary, quantum Hall effect is associated with two-dimensional electronic states in strong magnetic fields. In the semi-classical picture these states correspond to the "whispering gallery" trajectories (edge states in quantum mechanics), grazing the sample's boundary, so that in some geometries 
(e.g. a disk with a hole threading through the middle), this results in an effective ring topology which may lead to an Aharonov-Bohm type of oscillations in multiplyconnected samples. From another point of view to investigate these two effects, Aharonov-Bohm effect is a weak-field effect while quantum Hall effect is a strongfield one.

In this chapter, we discuss the physics of quantum Hall effect, to which more attention is paid, and Aharonov-Bohm effect. We first recall the basic physical concepts of Hall effect, classical and quantum definitions of Hall resistance are then introduced. The materials given next are the discovery of quantum Hall effect and different theoretical explanations of it. This is followed by a detailed discussion of Aharonov-Bohm effect. Finally, to illustrate the relation between them, we discuss a multiply-connected conduction problem.

\subsection{Basic Concepts of Hall Effect}

The discovery of quantum Hall effect is based on the following two basic conditions: first, low temperature and high magnetic field; second, two dimensional electronic system. The magnetic field which is perpendicular to the 2DEG system quantizes the motion of electrons in the plane so that the system is actually pseudo-zero dimensional [37]. In this situation the spectrum of a single particle turns into a series of highly degenerate Landau levels. However, disorder and interaction between clectrons can decrease the degeneracy and expand the Landau energy levels into energy sub-bands. All of above have been well known before the discovery of quantum Hall effect.

Before further discussion, it is necessary to recall the basic concepts of Hall effect. Hall resistance is one of the physical quantities which are usually measured in solid state physics. When a magnetic field is applied the conductivity $\sigma$ and resistivity $\rho$ become tensors,

$$
\sigma=\left(\begin{array}{ll}
\sigma_{x x} & \sigma_{x y} \\
\sigma_{y x} & \sigma_{y y}
\end{array}\right), \quad \rho=\left(\begin{array}{cc}
\rho_{x x} & \rho_{x y} \\
\rho_{y x} & \rho_{y y}
\end{array}\right) .
$$

Defining $\boldsymbol{J}$ and $\boldsymbol{E}$ as current density and electrical field strength respectively, then

$$
\boldsymbol{J}=\boldsymbol{\sigma} \cdot \boldsymbol{E}, \quad \boldsymbol{E}=\boldsymbol{\rho} \cdot \boldsymbol{J} .
$$


Considering the relaxation time $\tau$ that results from impurity scattering and Lorentz force, the "Langevin equation" of average electronic motion is

$$
\dot{v}=\frac{e}{m}\left(E+\frac{v \times B}{c}\right)-\frac{v}{\tau} .
$$

For a steady-state, the relation of current density $\boldsymbol{J}$ and average electron velocity $\boldsymbol{v}$ is known as

$$
\boldsymbol{J}=-n e v
$$

For simplicity, magnetic field is assumed along the $z$-axis, and therefore the equation of motion of electrons in the $x y$ plane becomes

$$
\begin{gathered}
\sigma_{0} E_{x}=\omega_{c} \tau j_{y}+j_{x} \\
\sigma_{0} E_{y}=-\omega_{c} \tau j_{x}+j_{y}
\end{gathered}
$$

where

$$
\sigma_{0}=\frac{n e^{2} \tau}{m}
$$

is just the classical conductivity in the absence of a magnetic field and $\omega_{c}$, known as the cyclotron frequency, is given by

$$
\omega_{c}=\frac{e B}{m c} \text {. }
$$

From Eqs. (2.1), (2.2), (2.3) and (2.4), it is easy to obtain

$$
\begin{gathered}
\rho_{x x}=\rho_{y y}=1 / \sigma_{0}, \quad \rho_{x y}=-\rho_{y x}=\omega_{c} \tau / \sigma_{0}, \\
\sigma_{x x}=\sigma_{y y}=\frac{\sigma_{0}}{1+\left(\omega_{c} \tau\right)^{2}}, \quad \sigma_{x y}=-\sigma_{y x}=\frac{-\sigma_{0} \omega_{c} \tau}{1+\left(\omega_{c} \tau\right)^{2}} .
\end{gathered}
$$

On the other hand, Eqs. (2.5), (2.6) and (2.7) give us

$$
\sigma_{x y}=-\frac{n e c}{B}+\frac{\sigma_{x x}}{\omega_{c} \tau}
$$

When $\sigma_{x x}=0$, we have $j_{x}=\sigma_{x y} E_{y}$ and $\sigma_{x y}$ is given by the first term of Eq. (2.8), i.e., the Hall conductivity is

$$
\sigma_{H}=\sigma_{x y}=-\frac{n e c}{B}
$$


Eq. (2.9) is still valid when we consider a quantum mechanical case. If we use Landau gauge, vector potential $\boldsymbol{A}$ is of the form, $\boldsymbol{A}=(B y, 0)$. The Hamiltonian of this system is then

$$
H=\frac{1}{2 m}\left[\left(p_{x}+\mu_{0} e B y\right)^{2}+p_{y}^{2}\right]+e E y,
$$

where the electrical field $E$ is along the $y$ direction. Writing the form of wave function
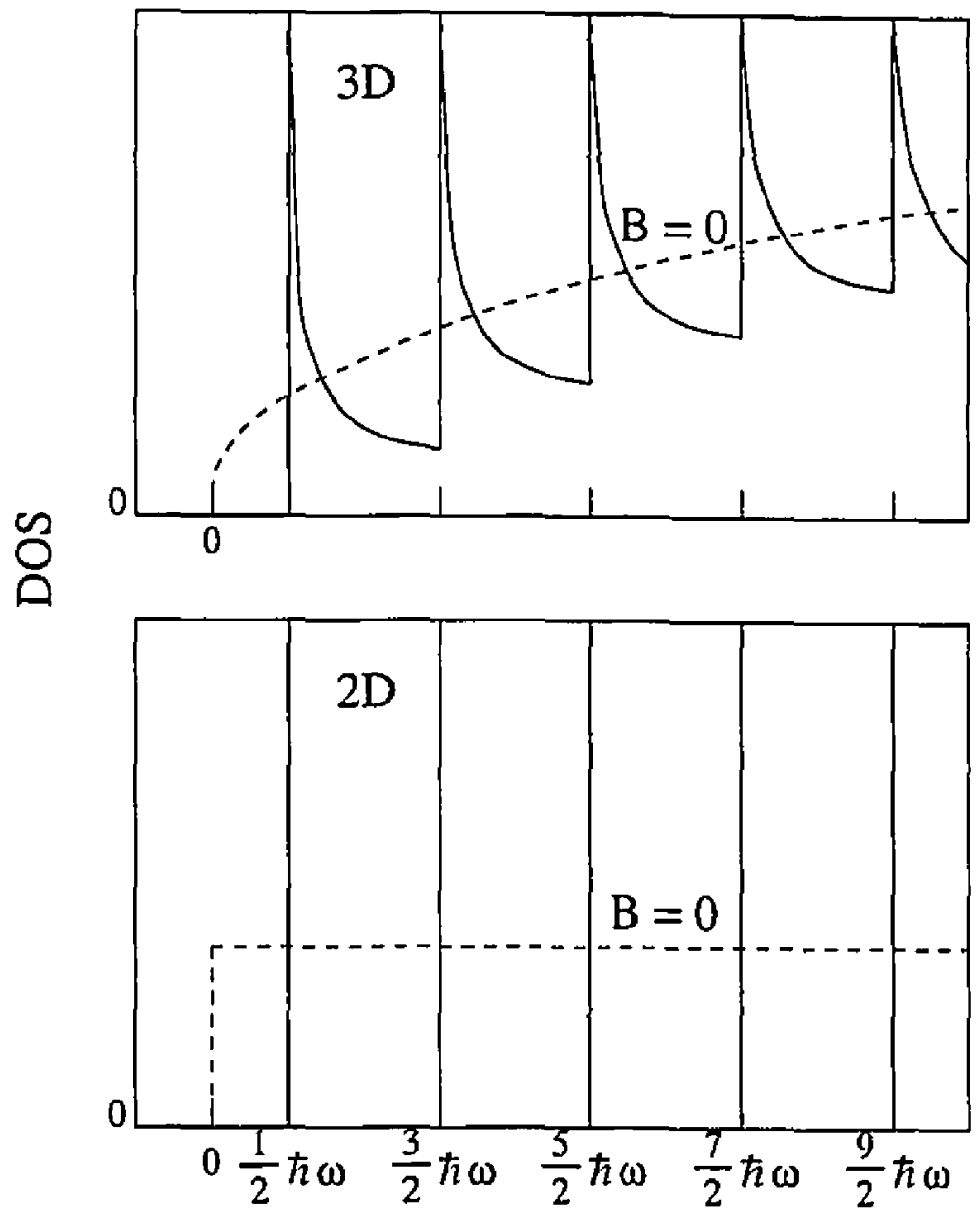

$\varepsilon$

Figure 2.1: Density of states of 2DEG and 3DEG in magnetic field.

as $\psi(x, y)=e^{-i k x} \phi(y)$, the Schrödinger equation becomes

$$
\left[-\frac{\hbar^{2}}{2 m} \frac{d^{2}}{d y^{2}}+\frac{1}{2} m \omega_{c}^{2}\left(y-l_{B}^{2} k\right)^{2}+e E y\right] \phi(y)=\varepsilon \phi(y)
$$


where

$$
l_{B}=\sqrt{\frac{\hbar c}{e B}}
$$

is the magnetic length. Making use of the formulae for a harmonic-oscillator, we will get the energy eigenvalue $\varepsilon_{i}$ and the wave function $\psi_{i}(x, y)$ of the $i$ th Landau level,

$$
\begin{gathered}
\varepsilon_{i}(E)=\left(i+\frac{1}{2}\right) \hbar \omega_{c}+e E\left(l_{B}^{2} k-e E / 2 m \omega_{c}^{2}\right), \\
\psi_{i}(x, y)=e^{-i k x} \exp \left[-\left(y-y_{0}\right)^{2} / 2 l_{B}^{2}\right] H_{i}\left[\left(y-y_{0}\right) / l_{c}\right],
\end{gathered}
$$

where $i=0,1,2,3, \cdots ;$ and $y_{0}=l_{B}^{2} k-e E / m \omega_{c}^{2}$. The effect of electrical field $\boldsymbol{E}$ does not change the structural features of energy spectrum, but only lets the eigenvalues have a shift.

Here we can see that the perfectly separated energy spectrum of 2DEG system in the presence of a magnetic field is very important for the occurrence of quantum Hall effect. On the contrary, electrons can freely move along the magnetic field if we consider the three dimensional case. So the density of state (DOS) will be a band in 3D (see Fig. (2.1)). Using the properties of harmonic-oscillator wave function we can obtain the average velocity

$$
<v_{x}>=\frac{1}{m} \int \psi_{i}^{*}\left(\frac{\hbar}{i} \frac{\partial}{\partial x}+\frac{B y}{c}\right) \psi_{i} d r=-E c / B .
$$

It is easy to get electrical current density $j_{x}=n e c E / B$, which is the same as the classical counterpart (using Eq. (2.9)).

\subsection{Discovery of Quantum Hall Effect}

The states of an electron are continuous in the $x y$ plane when there is no magnetic field. It is easy to find the density of states per unit area $g(E)=m / 2 \pi \hbar^{2}$. Once a magnetic field is present, the energy spectrum shrinks into equally separated Landau levels. Due to the conservation of the sum of states, every Landau level is degenerate which includes $\hbar \omega_{c} g(E)=e B / h c$ sates. We can obtain this degeneracy through another more direct picture: an electron has a cyclotron radius $r_{c}$ within a magnetic field, therefore for a $2 D E G$ system of unit area, it acquires a degeneracy for every Landau level, which is $1 / 2 \pi r_{c}^{2}=e B / h c$. 
We can prove that the current along the electrical field is zero by the similar method for getting Eq. (2.15). It is worth noting that the above conclusions have nothing to do with the energy level index $i$. If electrons fill up to the $i$ th Landau level and electron density is $n=i e B / h c$, then

$$
\sigma_{H}=n \frac{e}{B}=-i \frac{e^{2}}{h}
$$

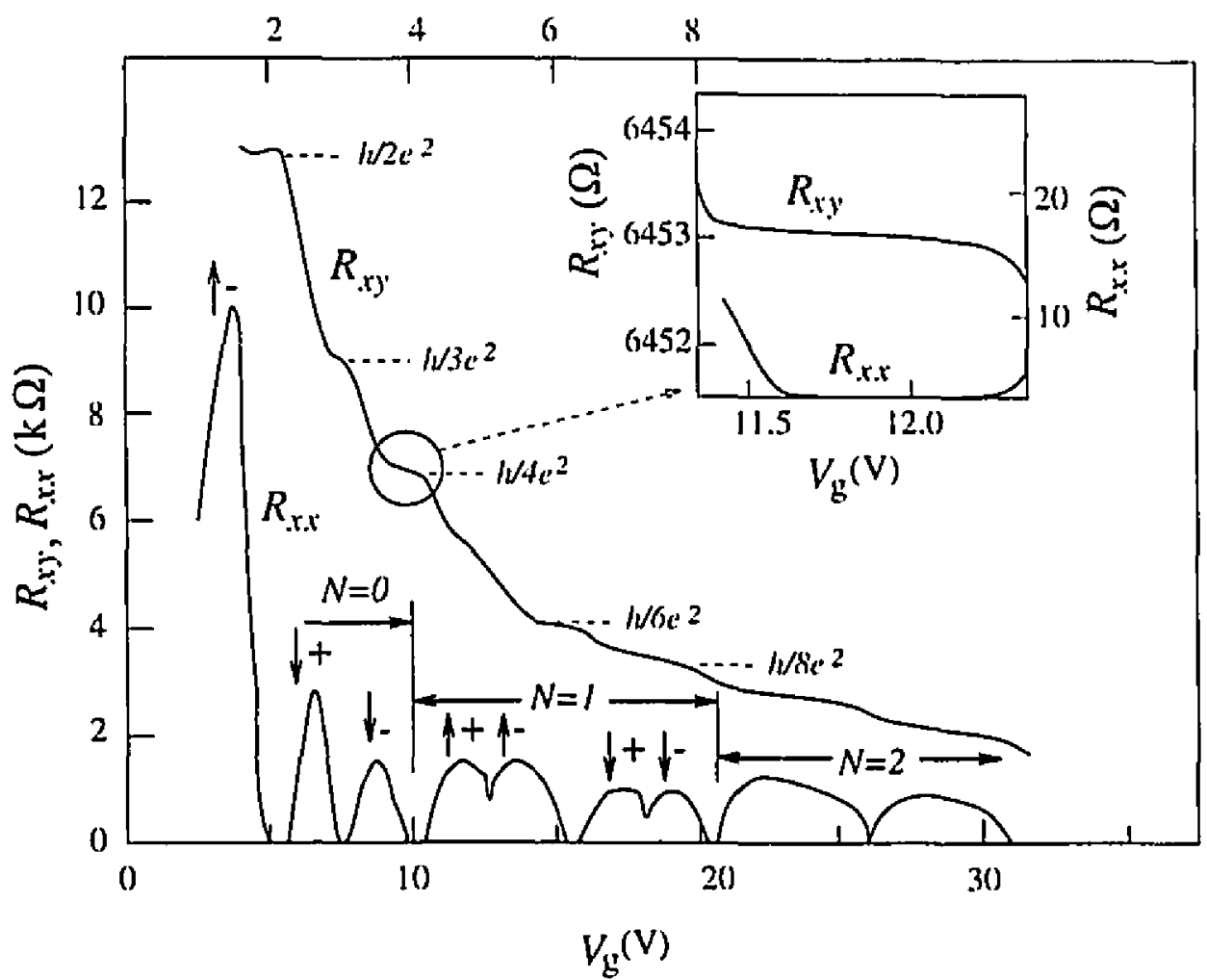

Figure 2.2: Quantum Hall effect observed on the inversion layer of Si-MOSFET. From Ref. [7].

Here we must emphasize that even though $i$ is an integer, Hall conductivity is actually proportional to $n$, the electron density. The second equal sign of Eq (2.16) is only valid for special $n$. For the inversion layer of Si-MOSFET, electron density $n$ is proportional to the grid voltage $V_{g}$, i.e., there is a linear relation between Hall conductivity and $V_{\theta}$. However von Klitzing et al. discovered an amazing phenomenon in 1980 that under the conditions of low temperature $(1.5 \mathrm{~K})$ and high magnetic field(18T), Hall conductivity $\sigma_{x y}$ has quantized plateaus and longitudinal conductivity $\sigma_{x x}$ vanishes $[7,38]$. This is so-called quantum Hall effect. Fig. (2.2) is the typical experimental result of quantum 
Hall effect. The appearance of Hall plateaus depends on the filling factor of the Landau levels,

$$
\nu=\frac{n}{\hbar \omega_{c} g(E)}=\frac{n c}{e B / h}=\frac{n}{B / \phi_{0}},
$$

where $\phi_{0} \equiv h c / e$ is the flux quantum. Eq. (2.17) clearly shows that the filling factor $\nu=$ electron density/flux quantum.

Quantum Hall effect is quite universal for 2DEG system, i.e., it only depends on several conditions such as magnetic field $B$, suitable disorder and is independent of materials.

\subsection{Theoretical Explanations of Quantum Hall Effect}

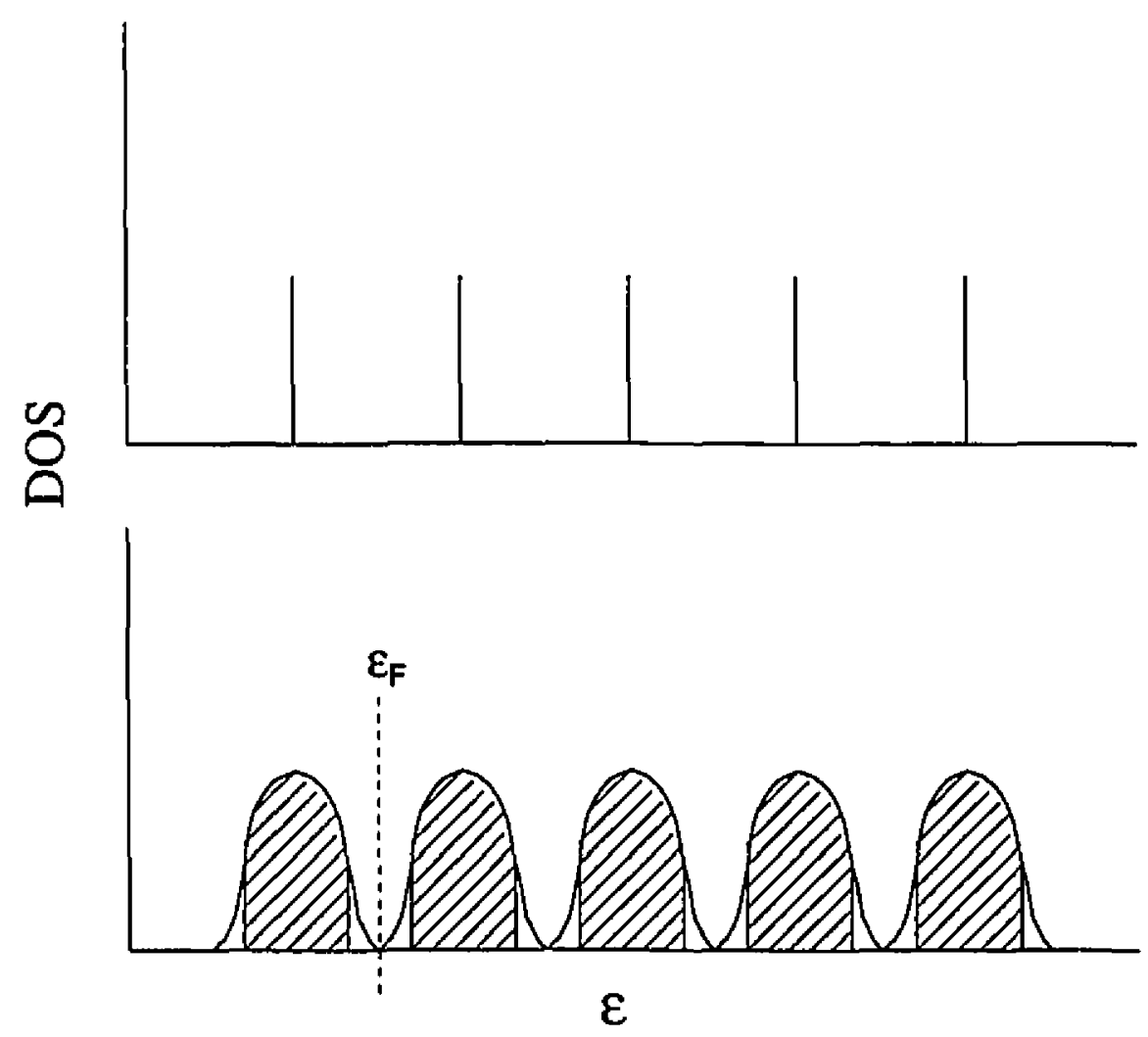

Figure 2.3: Density of states without (top) and with (bottom) disorder. Regions of dislocalized states are shaded. The dashed line indicates the Fermi level.

Experimental results showed that every : vak of longitudinal resistivity $\rho_{x x}$ is in the middle of a Landau level (see Fig. (2.2)). That the appearance of plateaus of $\rho_{x y}$ or 
$\sigma_{x y}$ and the vanish of $\rho_{x x}$ or $\sigma_{x x}$ take place between two adjacent Landau levels means that electrons are in localized states (hence they do not contribute to conduction $\sigma_{x x}$ ). On the other hand, when $\sigma_{x y}$ increases, clectrons are mobile, i.e., they are in extended states. Localization is necessary for quantum Hall effect. In §. 2.2, Eq. (2.11) is valid only for a perfect 2DEG system. However, in a real 2DEG system, disorder which comes from impurities and roughness of surfaces is present. Hence there must be a term of disorder potential $V(r)$ in the Hamiltonian, which brings a decrease to the degeneracy of Landau levels. In other words, density of state is no longer a sequence of $\delta$ functions. Every Landau level expands into a Landau sub-band with finite width $\Gamma$, and its property depends on the disordered potential. In Fig. (2.3), we can see that extended states are located near Landau levels whereas the localized state is in the middle of adjacent Landau levels. The existence of localized state is helpful for explaining quantum Hall effect. When electronic density increases or magnetic field decreases, localized state is gradually filled. However the occupation number of extended states is a constant and consequently Hall conductivity is a constant. The longitudinal conductivity tends to vanish at low temperature, nevertheless when Fermi energy goes through the center of the extended region, longitudinal conductivity is obviously nonzero and Hall conductivity jumps from one plateau to another.

\subsubsection{Laughlin's Explanation}

An elegant theory for quantum Hall effect was put forth by Laughlin [33], who demonstrated that it is in fact due to the long-range phase rigidity characteristic of a supercurrent, and that quantization can be derived from gauge invariance and the existence of a mobility gap.

We consider the situation illustrated in Fig. (2.4) where a ribbon of two-dimensional metal is bent into a loop of width $L$ and pierced everywhere by a magnetic field $\boldsymbol{B}_{\mathbf{0}}$ normal to its plane. The density of states of this system, shown in Fig. (2.3), consists, in the absence of disorder, of a sequence of $\delta$ functions, one for each Landau level and they broaden in the presence of disorder into bands of extended states separated by tails of the localized ones.

It will simplify our problem if we make use of Corbino plate (Fig. (2.4)) instead of the loop. These two models are of equivalence and coordinates $x$ and $y$ belong to a 
r' ght-angle coordinate system when radius $R$ is large enough. Consider the disordered case with Fermi level in a mobility gap. Hence there is a Hall current along the $y$ direction of the electrical field, and no current along the $x$ direction. If a state is extended, the magnetic flux $\phi$ penetrating through the closed orbit of an electron must be quantized (see next section)

$$
\pi R^{2} B_{0}=m_{1} \phi_{0}
$$

where $m_{1}$ is an integer and $\phi_{0} \equiv h c / e$ in the flux quantum.

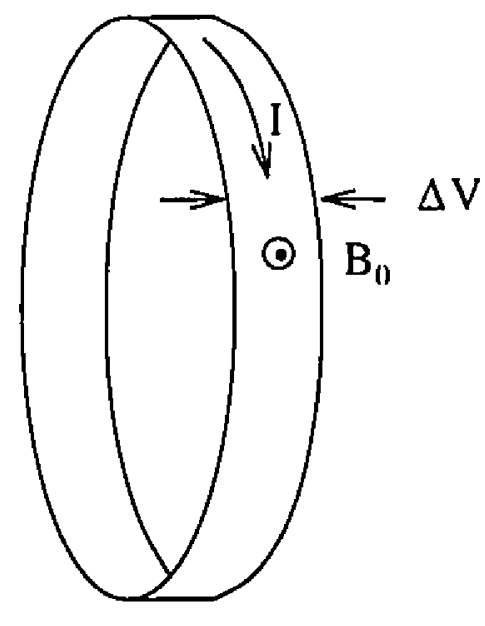

(a)

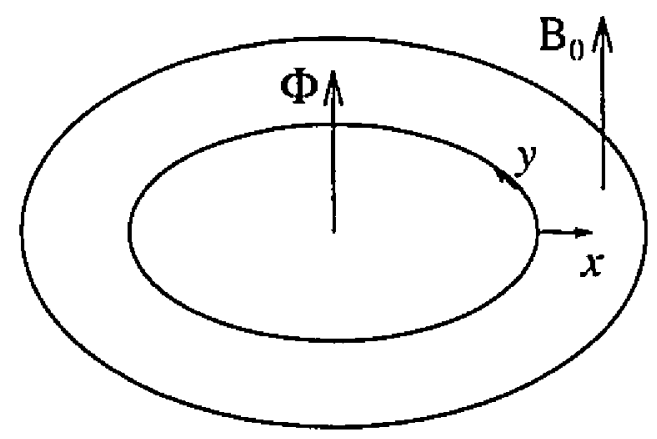

(b)

Figure 2.4: Left: Diagram of metallic loop. Right: Corbino plate.

In order to generate a Hall current, we set a solenoid threading through the middle of the plate with a magnetic flux $\Phi$ confined within it. Since there is no magnetic field out of the solenoid, it has no effect on a classical electron. However from the quantum mechanics point of view, the magnetic flux $\Phi$ plays an important role in affecting electrons through the vector potential $\boldsymbol{A}$. This is Aharonov-Bohm effect (we will discuss this effect in $\S 2.5$ ). Although Schrödinger equation will include $\boldsymbol{A}$, we can get rid off it by choosing the following gauge transformation,

$$
\psi(\theta) \longrightarrow \psi(\theta) \exp \left[i\left(\Phi / \phi_{0}\right) \theta\right]
$$


Whenever an electron goes back to the starting point after twinning the plate, $\psi$ becomes

$$
\psi \longrightarrow \psi \exp \left[2 \pi i \Phi / \phi_{0}\right] .
$$

That the wave function should be single-valued requires

$$
\Phi=m_{2} \phi_{0},
$$

where $m_{2}$ is another integer. Thus the quantization condition is given by

$$
\pi R^{2} B_{0}+\Phi=m \phi_{0}, \quad m=m_{1}+m_{2} \text {. }
$$

It is obvious that, in Eq. (2.22), if $\Phi$ increases with a fixed $m$, the radius $R$ will definitely decrease, i.e., the electrons move inwards. Particularly, if magnetic flux $\Phi$ increases by a flux quantum $\phi_{0}$, electronic state will switch to the $\left(m_{1}-1\right) t h$ state. The distance $L$, over which an electron moves from the outer side to the inner side of the plate, will results in a energy shift $\Delta \varepsilon=e E L$, provided that an electrical field $\boldsymbol{E}$ is along the $x$ direction.

We wish to relate the total current density $j_{y}$ carried around the loop to the potential drop $V$ from one edge to another. This current is equal to the adiabatic derivative of the total electronic energy $\varepsilon$ of the system with respect to the magnetic flux $\Phi$ through the loop (this can easily be shown by writing explicitly $\varepsilon=\langle H\rangle$ ),

$$
j_{y}=\frac{c}{L} \frac{\partial \varepsilon}{\partial \Phi} \text {. }
$$

Replacing $\partial \varepsilon / \partial \Phi$ by $\Delta \varepsilon / \phi_{0}$, we find

$$
j_{y}=\frac{e^{2}}{h} E .
$$

Since each of the $n$ energy levels contributes a current as Eq. (2.24), the total current density becomes

$$
j_{y}=n \frac{e^{2}}{h} E
$$

It is easy to obtain

$$
\sigma_{H}=-n \frac{e^{2}}{h}
$$

To explain quantum Hall effect, it is very important to assume that both localized and extended states exit, where extended states mean that electrons moving around the Corbino plate whereas localized states represent electrons coiling around impurities. 


\subsubsection{Halperin's Explanation}

Following the method of Laughlin [33], Halperin discussed some curious properties of clectronic states in a magnetic field which were implied by Laughlin's analysis. He found that states at the perimeter of the sample are quasi-one dimensional states which carry a current, and which do not become localized in the presence of a disordered potential of moderate strength. These so-called edge states play an important role in the Hall measurement, if the Fermi levels are different at two edge of the sample.

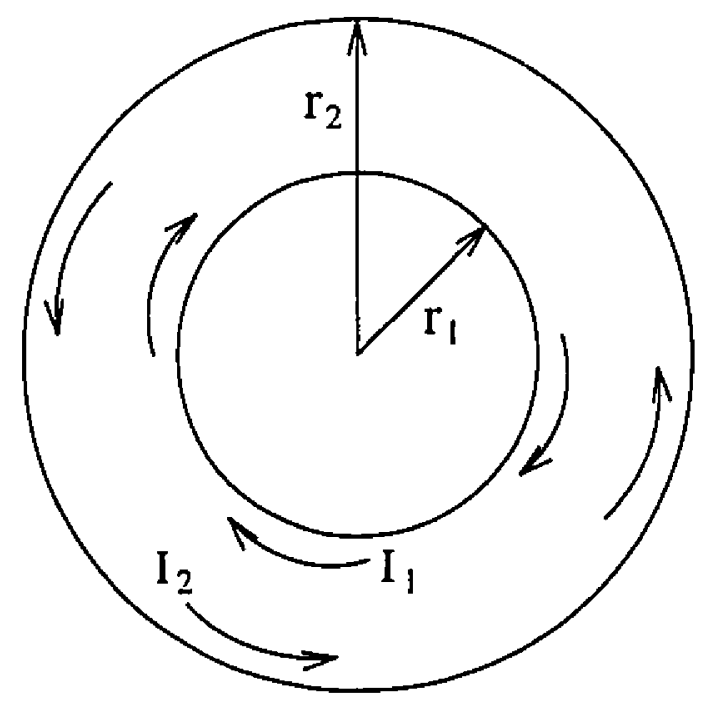

Figure 2.5: Geometry of sample. Annular film, region $r_{1}<r<r_{2}$ is placed in uniform magnetic field $B_{0}$, pointing out of the page. Additional magnetic flux $\Phi$ is confined to region $r<r_{1}$. Curved arrows show direction of currents $I_{1}$ and $I_{2}$ at the boundaries of film.

Let us consider a collection of noninteracting electrons, confined in an ideal uniform film of annular geometry, with a uniform magnetic field $\boldsymbol{B}_{\mathbf{0}}$ perpendicular to the plane of the sample (See Fig. (2.5)). We assume in addition that there is a magnetic flux $\Phi$, confined to the interior of a solenoid magnet threading the hole in the annulus, and we shall be able to vary the flux $\Phi$ without changing the magnetic field in the region where the electrons are confined. We shall assume that no electric field is present so that electrons feel a constant electrostatic potential in the interior of the film, and we assume that the dimensions of the annulus are very large compared to the cyclotron 
radius $r_{c}$ for electrons in the magnetic field. We adopt the gauge where the vector potential $\boldsymbol{A}$ points in the azimuthal $(\theta)$ direction, and the magnitude of $\boldsymbol{A}$ depends only on the distance from the center of the annulus:

$$
|A|=\frac{1}{2}\left|B_{0}\right| r+\Phi / 2 \pi r .
$$

Away from the edges of the film, the electronic states in this geometry have the form

$$
\psi_{m, \nu}(r) \sim e^{i m \theta} f_{\nu}\left(r-r_{m}\right),
$$

where $m$ and $\nu$ are integers with $\nu \geq 0, f_{\nu}$ is the $(\nu+1)$ th eigenstate of a onedimensional harmonic oscillator, and the radius $r_{m}$ is determined by

$$
B_{0} \pi r_{m}^{2}=m \phi_{0}-\Phi \text {. }
$$

Here $\phi_{0}$ is the flux quantum, $h c / e$. The width of $f$ is of order $r_{c}$, where $r_{c}$ is the cyclotron radius. Eq. (2.28) is only applicable in the interior of the annulus, i.e., if $r_{m}$ is in the range $r_{1}<r_{m}<r_{2}$, with $r_{m}-r_{1}$ and $r_{2}-r_{m}$ large compared to $r_{c}$. We shall assume throughout that $r_{c}$ is small compared to $r_{1}$ and $r_{2}-r_{1}$. The energies of the states (2.28) are given by the following formula

$$
\varepsilon_{m, \nu}=\hbar \omega_{c}\left(\nu+\frac{1}{2}\right)
$$

where $\omega_{c}$ is the cyclotron frequency determined by Eq. (2.6).

The electron density $\left|\psi_{m, \nu}(r)\right|^{2}$ associated with Eq. (2.28) is symmetric about the radius $r_{m}$, and decays rapidly for $\left|r-r_{m}\right| / r_{c} \gg 1$. The current carried by the state is given by

$$
I_{m, \nu}=\frac{e}{m^{*}} \int_{0}^{\infty} d r\left|\psi_{m, \nu}(r)\right|^{2}\left[\frac{m \hbar}{r}-\frac{e A(r)}{c}\right] \simeq \frac{e^{2} B_{0}}{m^{*} c} \int_{0}^{\infty} d r\left|\psi_{m, \nu}(r)\right|^{2}\left(r_{m}-r\right)
$$

where, $m^{*}$ is the carrier effective mass. The integral may be taken over the radial coordinate $r$ at any fixed value of $\theta$. From Eq. (2.31) the net current vanishes for states in the interior of the annulus, since the probability densities of the harmonic oscillator states are symmetric about the point $r=r_{m}$.

The situation is very different when $r_{m}$ is closer than a few times $r_{c}$ to an edge of the sample. Then the condition that the wave function vanishes at the edges of 
the sample will shift the energies of the eigenstates away from the Landau energies Eq. (2.30).

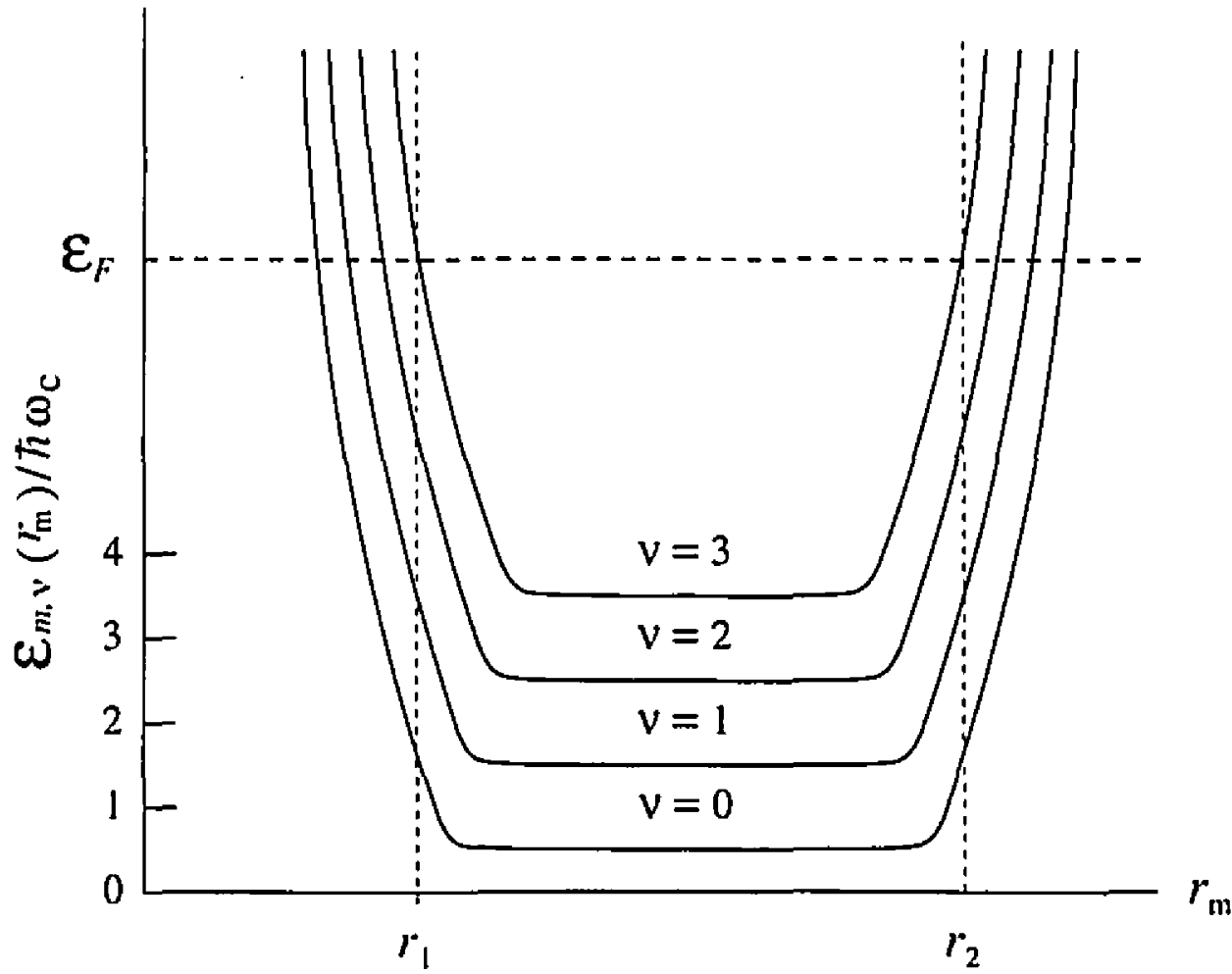

Figure 2.6: Quantum channels in high magnetic field for a perfect conductor. $r_{m}$ is the center of the wave function $\psi_{m, v}$ provided that $r_{m}$ is not too close to the boundary $r_{1}$ or $r_{2}$. Each bulk Landau level (flat portion of the dispersion in the center of the strip) gives rise to a branch of edge states near the sample boundary.

Let us focus our attention on the behavior near the outer edge of the annulus, and let us continue to use the index $\nu$ to label the number of nodes in the radial wave function. We may then write the electronic wave functions as

$$
\psi_{m, \nu}(r) \sim e^{i m \theta} g_{\nu}\left(r-r_{m}, r_{2}-r_{m}\right)
$$

where $g_{\nu}(x, s)$ is a wave function which is defined in the region $-\infty<x<s$ and has $\nu$ nodes, which vanishes for $x \rightarrow s$ and $x \rightarrow-\infty$, and which obeys the eigenvalue equation

$$
\left[-\frac{\hbar^{2}}{2 m^{*}} \frac{d^{2}}{d x^{2}}+\frac{B_{0}^{2} e^{2} x^{2}}{2 m^{*} c^{2}}\right] g_{\nu}=\varepsilon g_{\nu}
$$


From this equation it is clear that the eigenvalue $\varepsilon_{m, \nu}$ will approach the value $\varepsilon_{\nu}=$ $\hbar \omega_{c}\left(\nu+\frac{1}{2}\right)$, for $r_{2}-r_{m} \gg r_{c}$. The energy $\varepsilon_{m, \nu}$ will increase monotonically as $r_{m}$ increases, passing through the value $\varepsilon_{m, \nu}=\hbar \omega_{c}\left(\nu+\frac{3}{2}\right)$, when $r_{m}=r_{2}$, and increasing eventually as $\left(r_{m}-r_{2}\right)^{2} e^{2} B_{0}^{2} / 2 m^{*} c^{2}$ for $r_{m}-r_{2}>r_{c}$. The energy curve is sketched in Fig. (2.6).

Since the density $\left|\psi_{m, \nu}(r)\right|^{2}$ is no longer symmetric about $r=r_{m}$ near the edges, we no longer expect that $I_{m, \nu}=0$. In fact, making use of Eq. (2.29), it is readily established that

$$
I_{m, \nu}=-c \frac{\partial \varepsilon_{m, \nu}}{\partial \Phi}=\frac{e}{h} \frac{\partial \varepsilon_{m, \nu}}{\partial m}
$$

For $B_{0}>0$, we find that $I_{m, \nu}>0$, for $r_{m} \simeq r_{2}$, while $I_{m, \nu}<0$, near the inner edge $r_{m} \simeq r_{1}$.

Note that the quantity $\left|\partial \varepsilon_{m, \nu} / \partial m\right|$ is just the energy separation between adjacent energy levels for a given quantum number $\nu$. Thus the total current carried by states of a given $\nu$ in a small energy interval $\delta \varepsilon$ is equal to $(e / h) \delta \varepsilon$ at the outer edge of the sample, and $-(e / h) \delta \varepsilon$ at the inner edge.

Let us suppose that the Fermi level lies in between the energies $\varepsilon_{\nu}$ of two Landau levels $\nu=n-1$ and $\nu=n$, in the interior of the sample. Suppose also that near $r_{2}$ and $r_{1}$ there are Fermi levels $\varepsilon_{F}^{(2)}$ and $\varepsilon_{F}^{(1)}$, respectively, which differ from each other, but still lie in the interval between $\varepsilon_{n-1}$ and $\varepsilon_{n}$. If we denote the Fermi level difference, $\varepsilon_{F}^{(2)}-\varepsilon_{F}^{(1)}$, by $e \Delta$, then the total current carried by the edge states between $\varepsilon_{F}^{(2)}$ and $\varepsilon_{F}^{(1)}$ is clearly given by

$$
I=n e^{2} \Delta / h
$$

which is just the expression of quantum Hall effect.

The electronic states in the interior correspond to carriers describing a cyclotron motion with a guiding center that is stationary. Near the perimeter of the sample, each Landau level is associated with a state that is a strong function of position. These states correspond to carriers skipping along the boundary of the sample and are called edge states. Essentially, Halperin's explanation of the quantum Hall effect is based on this notion of edge states. 


\subsection{Aharonov-Bohm Effect}

The wave function of an electron comprises two parts: amplitude $c(r)$ and phase $\phi ;$ i.e., $\Psi=c(r) \exp (i \phi)$. The quantum interference among electrons which have traveled through the sample via all the conduction paths available in a device has been observed directly in recent experiment at very low temperature. Electron wave enter the device in phase, but upon reaching the other end, have been phase-shifted relative to each other because of collisions with defects along the way. If phase memory is maintained $[11,39]$ along each path, then the current exiting the device is a superposition of the wave function in all the paths.

A magnetic field can be used to tune the phase of the electronic wave function. An intriguing result of the relation between the wave function and the magnetic vector potential $\boldsymbol{A}$ (the relationship, loosely speaking, is the quantum-mechanical analogue of the Lorentz force) was pointed out by Aharonov and Bohm in 1959 [6].

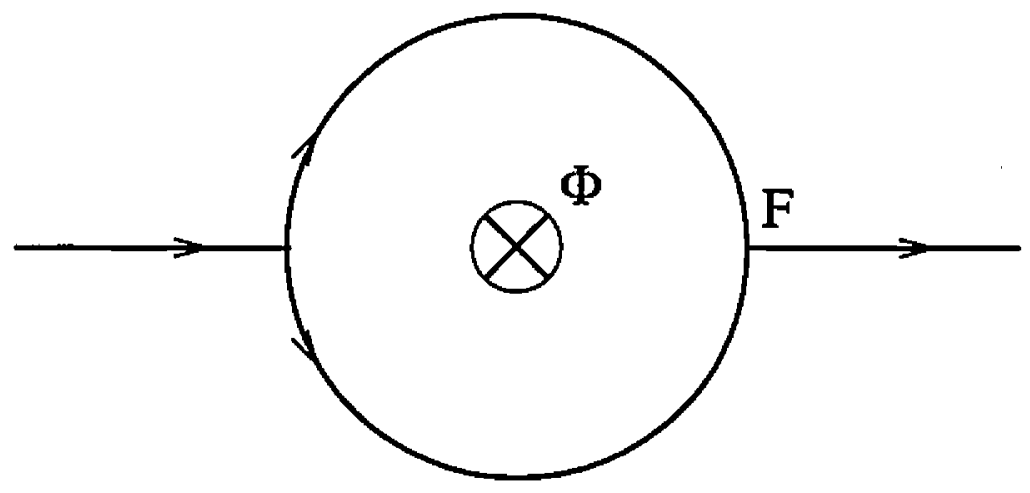

Figure 2.7: Schematic illustration of the geometry which results in periodic Aharonov-Bohm oscillations. A coherent beam enters from the left, splits around a magnetic flux $\Phi$ and recombines. The intensity of the current exiting to the right oseillates with period $\phi_{0} \equiv h c / e$.

Let us consider the case in Fig. (2.7). Using a very closely wound cylindrical solenoid of radius $R$ centered at the origin and with its axis in the $z$ direction, we create a magnetic field $\boldsymbol{B}$ which is essentially confined within the solenoid. However, the vector potential, $\boldsymbol{A}$, evidently, cannot be zero everywhere outside the solenoid, 
because the total flux through every circuit containing the origin is cqual to constant

$$
\Phi=\oint B \cdot d s=\oint A \cdot d l \text {. }
$$

To demonstrate the effects of the total flux, we begin with a coherent beam of clectrons. The beam is split into two parts, each going on opposite sides of the solenoid but avoiding it. The beam is recombined together at point $F$ (Fig. (2.7)). The Hamiltonian for this case is

$$
H=\frac{[\boldsymbol{P}-(e / c) \boldsymbol{A}]^{2}}{2 m}
$$

In singly connected regions, where $\boldsymbol{B}=\nabla \times \boldsymbol{A}=0$, we can always obtain a solution for the above Hamiltonian by taking $\psi=\psi_{0} e^{-i S / \hbar}$, where $\psi_{0}$ is the solution when $\boldsymbol{A}=0$ and where $\nabla S / \hbar=(e / c) A$. But in the imagined experiment discussed above, in which we have a multiply-connected region (the region outside the solenoid), $\psi_{0} e^{-i S / \hbar}$ is a non-single-valued function ${ }^{1}$ and therefore in general not a permissible solution of Schrödinger's equation. Nevertheless in our problem it is still possible to use such solutions because the wave function splits into two parts $\psi=\psi_{1}+\psi_{2}$, where $\psi_{1}$ represents the beam on one side of the solenoid and $\psi_{2}$ the beam on the opposite side. Each of these beams stays in a simply connected region. We therefore can writc

$$
\psi_{1}=\psi_{1}^{0} e^{-i S_{1} / \hbar}, \quad \psi_{2}=\psi_{2}^{0} e^{-i S_{2} / h}
$$

where $S_{1}$ and $S_{2}$ are equal to $(e / c) \int \boldsymbol{A} \cdot d l$ along the paths of the first and the second beam, respectively. Hence the total wave function at $F$ will be

$$
\psi=\psi_{1}^{0} e^{-i S_{2} / \hbar}+\psi_{2}^{0} e^{-i S_{2} / \hbar}
$$

and we can find the intensity,

$$
|\psi|^{2}=\left|\psi_{1}^{0}\right|^{2}+\left|\psi_{2}^{0}\right|^{2}+\operatorname{Re}\left(\psi_{1}^{0} \psi_{2}^{0}\right) \cos (\Delta S / \hbar)+\operatorname{Im}\left(\psi_{1}^{0} \psi_{2}^{0}\right) \sin (\Delta S / \hbar)
$$

where,

$$
\Delta S / \hbar=\left(S_{1}-S_{2}\right) / \hbar=(e / \hbar c) \oint \boldsymbol{A} \cdot d l=(e / \hbar c) \Phi
$$

1 Unless $\Phi=n h c / e$, where $n$ is an integer. 
is the phase difference of the two beams. The interference between the two beams will thus depend on this phase difference. The resulting current would be modulated periodically by the amount of flux and thus imposes a fundamental periodicity

$$
R(\Phi)=R(\Phi+n(h c / e)), \quad n=1,2,3, \cdots
$$

on the resistance $R(\Phi)$ as a function of the perpendicular magnetic field $\boldsymbol{B}$. This effect will exist, even if the magnetic field is completely confined to the interior of the path so that the electron always travels in a magnetic-field-free region. The existence of a vector potential $\boldsymbol{A}$ is all that is required to produce the phase shift. In a real experiment, however, the magnetic field penetrates the arms of the ring as well as its interior so that deviations from Eq. (2.42) can occur. Since in many situations such deviations are small at least at a limited field range, one still refers to magnetoresistance oscillation as an Aharonov - Bohm effect.

It is worthwhile to note that in the geometry of Fig. (2.7), the magnetic field $B$ is physically separated from the wave function. This spatial separation of the wave function and the field implies, in the framework of classical physics, that there can be no effect on the particle represented by the wave function. From this argument, the Aharonov-Bohm effect is said to demonstrate that the magnetic vector potential is a real physical potential, not simply a mathematical convenience.

\subsection{Relation of Two Effects}

So far the quantum Hall effect and Aharonov-Bohm effect have been discussed separately. However, using one conductor, we actually can observe both the quantum Hall effect and $A B$ effect by varying magnetic field or the size of the conductor.

To illustrate the dramatic change in current distribution and the backscattering that develops in an intense magnetic field, we examine the magnetoresistance of a prototypical scatterer - an annulus (see Fig. (2.8)) [40,41, 42]. Following the analysis given by Jain [43] and Büttiker [27, 44], we assume that the magnetic field penetrates both the wire comprising the annulus and the annulus itself. Technically, the "hard core" in annulus can be realized by an "antidot" (see below). In Fig. (2.8) the shaded area is the antidot. If $B e W_{a}^{2} / \hbar c \ll 1$, i.e., $l_{B} \gg W_{a}\left(l_{B}\right.$ is the magnetic 
length), the magnetoresistance is periodic in the flux $h c / e$ through the average area of the annulus due to the $A B$ effect. As represented in Fig. (2.8a), the AB effect occurs because two electron trajectories which encircle a magnetic flux acquire a relative phase shift proportional to the flux. As the flux changes, the transmission probability of an electron through the annulus oscillates with a periodicity of $h c / e$. In contrast, in the quantum Hall regime, the current is carried around the annulus by edge states, as shown in Fig. (2.8b). The AB effect is suppressed in the quantum Hall regime because:
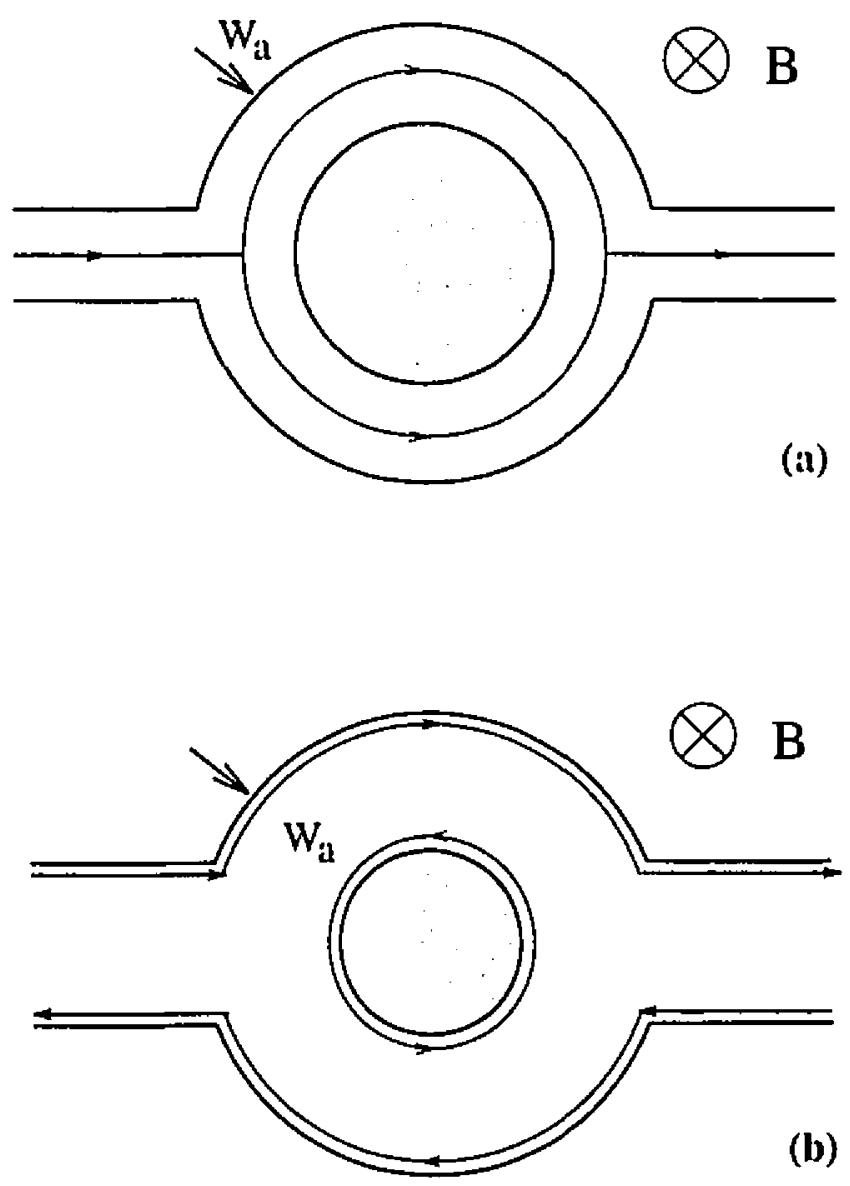

Figure 2.8: A schematic representation of transport through an annulus in the $A B$ effect regime (a), and in the quantum Hall regime (b). The arrows indicate the direction of current. In the quantized Hall regime, a net current results from the difference between two oppositely directed currents assocjated with the edge states. 
1. The outer edge states, which are connected to the probes and determine the resistance, do not enclose a flux.

2. The inner edge states that do enclose a flux are not coupled to the outer edge states.

3. Ideally, the edge states do not backscatter.

Since the magnetoresistance of the annulus oscillates periodically with magnetic field in the $A B$ transport regime, the magnetoresistance provides an unambiguous periodic signature of the backscattering as a function of the field. The observation of oscillations due to $\mathrm{AB}$ effect has been reported before $[40,41,42]$ in the magnetoresistance of annuli fabricated in high-mobility $\mathrm{AlGaAs} / \mathrm{GaAs}$ heterojunctions. Under the conditions of experiments, the magnetic field penetrated both the annulus and the $1 \mathrm{D}$ constrictions comprising the annulus. For magnetic field where $B e W_{a}^{2} / h c \gg 1$ (the quantum Hall regime), the oscillation amplitude was suppressed [41, 45]. The oscillations, with a periodicity near zero field corresponding to a flux of $h c / e$ through the average area of the annulus, decrease exponentially in intensity and shift to a lower frequency as the magnetic field increases $[40,46]$. The frequency of oscillation observed in a high magnetic field corresponds to a flux of $h c / e$ through the area circumscribed by the inside diameter of the annulus, and the exponential decrease in the amplitude is indicative of the exponential decrease in the overlap between the outside and inside edges of the annulus. These observations show that the net current is carried by edge states in the quantum Hall regime, and that the suppression of the $\mathrm{AB}$ effect is due to the suppression of backscattering.

The above discussion is for two extreme cases. It is natural to ask what would happen if $B$ and $W_{a}$ both are suitable values so that $B e W_{a}^{2} / \hbar c \sim 1$ ? Answering this question is the subject of this thesis which is presented in Chapter 4. 


\subsection{Introductory Remarks}

Over the past several years, a different picture of quantum Hall effect has emerged [22]. Instead of the closed conductors implicit in the topological explanations $[33,19]$ of the quantum Hall effect, which we discussed in Chapter 2, Büttiker considered another physical picture, shown in Fig. (3.1) . Consider a system where a number of probes are connected to the conductor that serve as carrier source, carrier sink, and serve

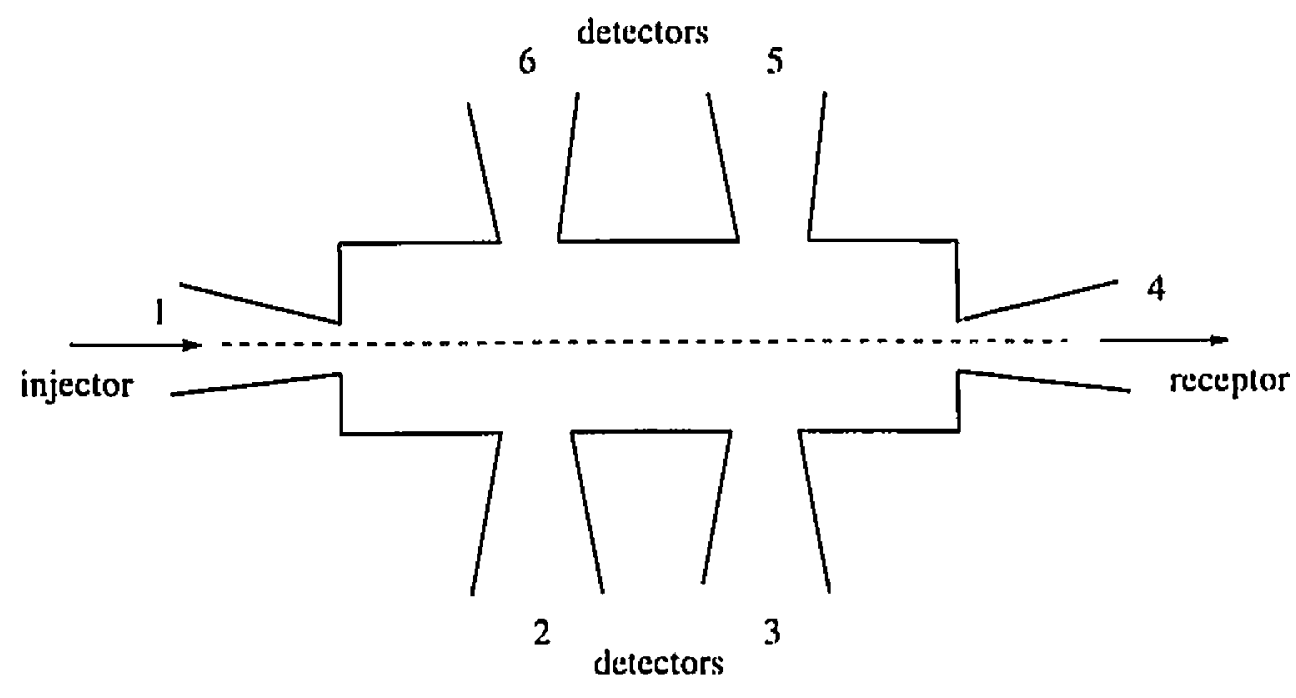

Figure 3.1: Conductor with a number of probes permitting carriers to enter and leave.

to attach voltmeters. The source plays a role as an injector of carriers and the sink as a receptor. The voltage probes are carrier detectors. Do we observe a quantum Hall effect quite independent of the properties of the injector and the detectors? 
The answer to this question is: No. The distribution of incident carriers into states of 2DEG depends on the properties of the source probe [22]. The memory of this initial distribution can be lost only through the action of inelastic events. Elastic scattering alone can lead to a modification of the incident distribution but not to an equilibration, i.e., it can only change the direction of the incident carriers. Therefore, if the injector and detector are close enough, the Hall resistance is not quantized unless the injection and detection proceeds according to very stringent conditions. This effect has been demonstrated in a clear manner in experiments by van Wees et al [47]. and Komiyama et al [48].

In this chapter, we discuss Büttiker's approach [22, 27] to electrical conduction that we employ later on in this thesis. We assume that the dimension of conductor, shown in Fig. (3.1), is less than the mean free path of the carrier thus we enter the quantum ballistic transport regime. In this regime it is the electron scattering at the conductor boundaries which limits the current, rather than the impurities scattering. So we assume that the motion of carriers from the entrance to the exit can be treated as a purely elastic scattering problem. Dissipation is assumed to happen only in the electron reservoirs which are connected to the probes. An electron wave incident in probe $j$ typically is associated with waves transmitted into all the other probes $i \neq j$ and a reflected wave in probe $j$. The conduction process is thus viewed as a transmission and reflection problem. The description of wave motion in terms of reflection and transmission coefficients is familiar from the propagation of electromagnetic waves in waveguides and has found wide application in this fields. It is also familiar in tunneling theory which calculates the resistance of tunneling devices in terms of tunneling matrix elements. Yet both of these stimuli did not lead to a transport theory of electric conduction in terms of transmission and reflection coefficients in the 1930s and 1940s. Papers that treat the conductor not only of a single scatterer but of an entire sample in terms of transmission and reflection probabilities seem to have appeared only with the work of Landauer in 1957 and in a more explicit way in 1970. Landauer's discussion of the resistance $[11,39]$ emphasizes the local electrostatic potential that arises due to charge accumulation and depletion at the scattering centers of the sample. More precisely, the voltage across a scat- 
terer is determined in portions of the sample a few screening lengths away from the scatterer. This leads to an electrostatic potential difference that is determined by a charge neutrality condition. Büttiker, together with Imry and Landauer, reestablished in 1985 a generalized resistance formula [49] that had been derived earlier by Azbel [50]. But none of these papers addressed the role of probes. The distinction between open and closed systems was emphasized in the early discussion of samplespecific interference effects in normal loops $[51,52,53,54]$. However, only the probes connected to the current source and the sink were treated explicitly. Eventually, it was recognized that a voltage probe, which permit no net carrier flux [55], nevertheless allows carrier exchange into and out of the conductor. The consequence is that a voltage probe permits phase randomization and, in the presence of transport, dissipation of energy [54]. Thus, voltage probes provide a model of a dissipative scattering for electrical conduction $[49,56,57,58,59,60,61,62]$. These notions, amplified by a set of experiments by Benoit et al.[63], led to a formulation of resistances on an equivalent footing of all the probes [12, 27].

In contrast to the electrostatic potential at points inside a conductor, which essentially is determined by a charge neutrality condition, the consideration of a conductor including the probes leads to a formulation of resistances in terms of the chemical potentials of the electron reservoir. The measurement of a chemical potential is conveniently expressed in terms of a zero-current condition. Hall resistances and longitudinal resistances are measured in a four-terminal set-up: a carrier flux $I$ is driven from the current source probe $k$ to the current sink probe $l$ and the measured voltage is determined by connecting a pair of probes $m$ and $n$ to a voltmeter. The resistance measured in the configuration $m n k l$ is

$$
R_{k l, m n}=\frac{V_{m}-V_{n}}{I}
$$

If an imagination line is drawn from the carrier source to the carrier sink probe, the measured resistance is called a Hall resistance if the two probes are on opposite sides of this line and is called a longitudinal resistance if the probes are on the same side of this line. In Eq. (3.1), the potentials $e V_{m}$ characterize chemical potentials (Fermi energies) of the electron reservoirs connected to probes. 


\subsection{The Scattering Problem}

Consider a conductor, shown in Fig. (3.2), with several probes that permit the entrance or exit of carriers. To provide a mathematical formulation of a scattering

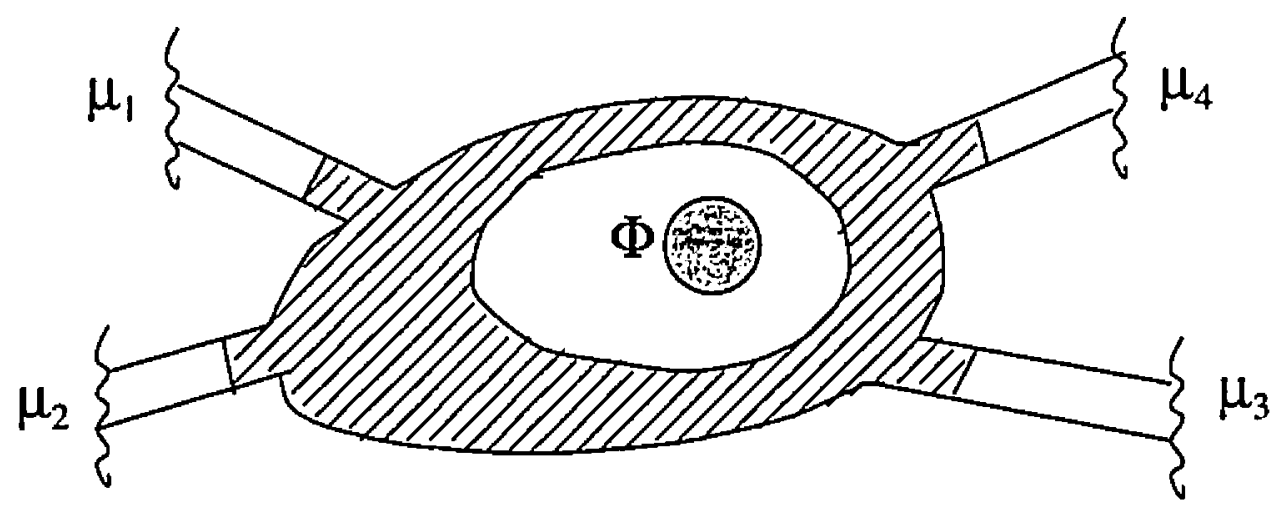

Figure 3.2: Conductor with probes connected to electron reservoirs at chemical potentials $\mu_{i}, i=$ $1,2,3,4$ and an Aharonov-Bohm flux $\Phi$.

problem, we use the notion of asymptotic regions which that permit the definition of incident and outgoing waves. This is achieved by assuming that each probe of the conductor in Fig. (3.2) eventually widens into a perfect probe. For mathematical purposes, it is assumed that such a perfect probe to extend uniformly for an infinite distance away from the conductor. This mathematical device clearly has no counterpart in the real physical world. Perfect wires do not exist. Therefore, this mathematical device makes sense only if our final result is largely independent of these assumptions. As we will show, this indeed is the case.

In perfect wires, the Hamiltonian is separable into a part that describes motion slong the perfect wire and a part that describes motion transverse to the perfect wire. Therefore, in an infinitely extended perfect wire, the wave functions are of the form,

$$
\psi_{i, \pm k}=e^{ \pm i k x}, i, \pm k(y)
$$

The factor $e^{ \pm i k x}$ is due to translational invariance along the wire, $f_{i, \pm k}$ is the transverse 
wave function. For fixed $k$, the spectrum consists of a ladder of discrete states with energy $E_{i}(k)$. The specific $k$ dependence of the dispersion $E_{i}(k)$ depends on the type of perfect conductor considered and depends on whether or not a magnetic field is present. The specific form of the dispersion is irrelevant, however, and our discussion in this section is completely general.

Suppose now that we have defined the properties of the asymptotic regions in all the probes connected to the conductor. Consider a wave of unit amplitude of the form given by Eq. (3.2), which is incident in probe $n$ and channel $j$. A solution of the scattering problem consists of this incident wave and of outgoing waves in (typically) all the other probes. The outgoing waves in the asymptotic region again are of the form given by Eq. (3.2) multiplied by a factor $S_{i j, m n}$, which accounts for the fact that current is conserved. The outgoing wave in channel $i$ in probe $m$ due to a wave of unit amplitude incident in probe $n$ in channel $j$ is

$$
\left(\frac{v_{j m}}{v_{i m}}\right)^{\frac{1}{2}} S_{i j, m n} e^{-i k_{i m} x} f_{i m}(y) \text {. }
$$

Here,

$$
v_{j n}=\left.\left(\frac{1}{\hbar}\right)\left(\frac{d E_{j n}(k)}{d k}\right)\right|_{E_{F}},
$$

is the velocity of carriers in probe $n$ and quantum channel $j$ at the Fermi energy $E_{F}$. The velocity factors in Eq. (3.3) are chosen such that the absolute square of the amplitude $S_{i j, m n}$ is the transmission probability $T_{i j, m n}$ (or the reflection probability $R_{i j, n n}$ ) of a carrier incident in probe $n$ and channel $j$ to exit in probe $m$ and channel $i$, (or incident in probe $n$ in channel $j$ and reflected into probe $n$ into channel $i$ ),

$$
T_{i j, m n}=\left|S_{i j, m n}\right|^{2}, \quad R_{i j, n n}=\left|S_{i j, n n}\right|^{2} .
$$

The incident wave function has an amplitude that is normalized to one and thus gives rise to an incident current $I_{j n}=v_{j n}$. The outgoing wave in probe $m$ and channel $i$ is associated with a current $I_{i m}=v_{i m}\left(v_{j n} / v_{i m}\right)\left|S_{i j, m n}\right|^{2}=v_{j n} T_{i j, m n}$. Alternatively, if the incident current is $I_{j n}=\left|\alpha_{j n}\right|^{2}$, then the outgoing currents are determined by the square of the amplitudes $S_{i j, m n} \alpha_{j n}$. The scattering matrix $S$ with the elements $S_{i j, m n}$ thus provides a linear relationship between the current amplitudes of the incident waves and the out going waves. Because current is conserved, $S$ must be a unitary 
matrix. Denoting the Hermite conjugation by $\dagger$, we must have $\boldsymbol{S}^{\dagger}=\boldsymbol{S}^{-1}$. Since the Hamiltonian describing the scattering at the sample is also invariant if we reverse the momenta and the magnetic field simultaneously, the scattering matrix must then have the property $\boldsymbol{S}^{*}(-\boldsymbol{B})=\boldsymbol{S}^{-1}(\boldsymbol{B})$. Here, the asterisk denotes complex conjugation. Taken together, these two conditions imply that the scattering matrix has the symmetry $S^{T}(B)=S(-B)$. Hence the amplitudes of the $S$ matrix obey a microreversibility condition, $S_{i j, m n}(B)=S_{j i, n m}(-\boldsymbol{B})$. The transmission (or reflection) amplitude of a carrier incident in probe $n$ in channel $j$ to exit in probe $m$ in channel $i$ in the presence of a magnetic field is equal to the amplitude of reciprocal process of a carrier incident in probe $m$ in channel $i$ to exit in probe $n$ in channel $j$ if the magnetic field is reversed. Thus, as a consequence of microreversibility, the transmission and reflection probabilities also obey the relations,

$$
T_{i j, m n}(B)=T_{j i, n m}(-B), \quad R_{i j, n n}(B)=R_{j i, n n}(-B)
$$

The microreversibility properties, Eq. (3.6), of the transmission and reflection probabilities are necessary to provide the reciprocity of resistances, but the microreversibility of the transmission probabilities is not a sufficient condition to arrive at the reciprocity of resistances. The reciprocity of resistances is a consequence of both microreversibility and equilibration provided by the electron reservoirs connected to the conductor.

\subsection{The Current Response due to Differing Chemical Po- tentials}

The mathematical formulation of the scattering problem given earlier needs to be augmented by physical considerations that specify the role of the reservoirs attached to the probes of the conductor. First, it is assumed that a carrier that approaches a reservoir from inside the conductor will enter the reservoir with probability one once it has reached the asymptotic regions discussed before. Scattering that the carrier suffers in the reservoir completely randomizes the energy and phase of the carrier such that a carrier entering the conductor bears no phase and energy relationship to an exiting carrier. Entering and leaving the reservoir are treated as irreversible events. 
Since the reservoirs connected to the probes are a source of irreversibility, it follows that waves incident from differing reservoirs cannot exhibit quantum interference effects. This allows us to treat waves incident from differing probes as incoherent. Second, we must specify how a reservoir attached to a probe populates the quantum channels (edge states). In part of the theory of electric conduction, sometimes it is assumed that the incident carrier stream is populated according to a shifted Fermi distribution. The result is that the quantum channels with low threshold energy, which have a total momentum parallel to the probe, are populated up to higher energies than quantum channels with high threshold energies, which have a small longitudinal momentum component. Such a non-equilibrium distribution of incident carriers does not give rise to the reciprocity of resistances. Rather, we assume that the reservoirs are at equilibrium and fill all the quantum channels of probe $m$ according to the Fermi function of reservoir $m$. The reservoir populates all quantum channels equivalently [49]. Next, we shall assume, for simplicity, that the tempcrature is so small that the Fermi function of reservoir $m$ can be described in terms of the chemical potential (the Fermi energy) $\mu_{m}$ of reservoir $m$. The equilibrium population of incident quantum channels simplifies the calculation considerably, and together with the microreversibility of the scattering matrix, ensures the reciprocity of the resistances.

Since the reservoirs feed all channels equally and up to their chemical potential, it is only the total transmission probabilities that are relevant. If each incident channel in probe $n$ supports a unit current $\left|\alpha_{j n}\right|^{2}=1$, the total current in probe $n$ is $\Sigma_{i j}\left|S_{i j, m n}\right|^{2}\left|\alpha_{j n}\right|^{2}=\Sigma_{i j}\left|S_{i j, m n}\right|^{2}$. Here, the sum is over all $M_{n}$ incident channels in probe $n$ and over all outgoing channels $M_{m}$ in probe $m$. Hence, transmission from probe $n$ into probe $m$ can be characterized by a total transmission probability [12],

$$
T_{m n}=\sum_{i=1}^{i=M_{m}} \sum_{j=1}^{j=M_{n}} T_{i j, m n} .
$$

Similarly, the total reflected current in probe $n$ due to carriers incident in probe $n$ can be characterized by a total probability for reflection,

$$
R_{n n}=\sum_{i=1}^{i=M_{n}} \sum_{j=1}^{j=M_{n}} R_{i j, n n} .
$$


Eqs. (3.7) and (3.8) are the relevant transport coefficients as we now will demonstrate.

Next, we have to evaluate the currents driven through a sample in the presence of small differences between the chemical potentials. To find the relation betwer in the currents and the chemical potentials, we proceed as follows. Denote the lowest of the chemical potentials $\mu_{m}$ by $\mu_{0}$. At energies smaller than $\mu_{0}$ all states are fully occupied. These states cannot contributes to a net current flow. Thus, the considerations can be limited to energies larger than $\mu_{0}$. Reservoir $n$ feeds all quantum channels in the energy range $\mu_{n}-\mu_{0}$. The current incident from this reservoir in channel $j$ in this energy interval is $I=e v_{j n}\left(d n_{j n} / d E\right)\left(\mu_{n}-\mu_{0}\right)$. For each of these quantum channels, the appropriate density of states is $d n / d k=1 / 2 \pi$, thus density of states with respect to energy is

$$
\frac{d n_{j n}}{d E}=\frac{d n}{d k} \frac{d k}{d E_{j n}}=\frac{1}{h v_{j n}} .
$$

Hence, the density of states is inversely proportional to the velocity of the carriers. Using (3.9), the reservoir $j$ feeds a current,

$$
I=\frac{e}{h}\left(\mu_{n}-\mu_{0}\right)
$$

into each of the $M_{n}$ incident quantum channels. Note that this current is quite independent of any particular properties of the system (magnetic field, effective mass, density of states...). The universality expressed by Eq. (3.10) is essential for the occurrence of quantized resistances.

It now is a simple matter to calculate the net currents flowing into the probes of a conductor. The total incident carrier flux in probe $m$ is $M_{m}\left(\mu_{m}-\mu_{0}\right) / h$. Of this flux, a portion $R_{m m}\left(\mu_{m}-\mu_{0}\right) / h$ is reflected. The incident flux is diminished further by carriers that are incident from the other probes and are transmitted into probe $m$ (because they flow in opposite directions). These fluxes are proportional to $T_{m n}\left(\mu_{m}-\mu_{0}\right) / h$. Thus, the net current in probe $m$ is [27]

$$
I_{m}=\frac{e}{h}\left[\left(M_{m}-R_{m m}\right) \mu_{m}-\sum_{n \neq m} T_{m n} \mu_{n}\right] \text {. }
$$

The reference chemical potential drops out as expected. Due to current conservation, we must have

$$
M_{m}=R_{m m}+\sum_{n \neq m} T_{m n} .
$$


Hence we can also express Eq. (3.11) in the form,

$$
I_{m}=\frac{e}{h} \sum_{n \neq m} T_{m n}\left(\mu_{m}-\mu_{n}\right) .
$$

Eqs. (3.12) and (3.13) are the important equations of this section. They represent a quantum mechanical version of the Kirchoft laws. These equations provide a linear relationship between chemical potentials of electron reservoirs and currents in the probes. It is a linear response relation. The transmission and reflection coefficients in Eqs. (3.12) and (3.13) are evaluated at equilibrium at the Fermi energy. The linear response coefficients $T_{m n}$ and $R_{m m}$ have the symmetry that, according to Onsager and Casimir [64], is required. The diagonal coefficients $R_{m m}$ are symmetric under flux reversal and the off-diagonal coefficients obey a reciprocity relation,

$$
R_{m m}(B)=R_{m m}(-B), T_{m n}(B)=T_{n m}(-B)
$$

The symmetry of the total transmission and reflection coefficients is a consequence of the symmetry of the individual transmission and reflection probabilities as stated by Eq. (3.6).

\subsection{Resistance and Transmission}

Using the relations between currents and chemical potentials, Eq. (3.11), we can now determine the resistance for a given configuration of current sources, sinks, and voltage probes. We discuss here two cases explicitly: the case of a two-probe conductor and the case of a resistance measurement in a four-probe setup.

If the conductor is connected only to two reservoirs with $M_{1}$ and $M_{2}$ quantum channels, current conservation as stated by Eq. (3.12) requires $M_{1}=R_{11}(B)+T_{12}(B)$ and $M_{2}=R_{2,2}(B)+T_{21}(B)$. Since the total reflection coefficients are symmetric under field reversal, it follows that $M_{1}=R_{11}(B)+T_{12}(-B)$ and $M_{2}=R_{22}(B)+$ $T_{21}(-B)$. Consequently, the two-terminal transmission probability $T_{21}=T_{12} \equiv T$ is also symmetric under field reversal. Thus, Eqs. (3.11) and (3.13) yield a two-probe resistance,

$$
R_{12,12} \equiv \frac{\mu_{1}-\mu_{2}}{e I}=\left(\frac{h}{e^{2}}\right)\left(\frac{1}{T}\right)
$$


Eq. (3.15) has been obtained by Fisher and Lee [65] in a linear response calculation that did not appeal to reservoirs and is contained in a number of papers as a limiting result $[49,66]$. The physical content of Eq. (3.15), which gives the resistance for current flow between two equilibrium reservoirs, was comprehended by Imry [10]. The comparison of the two-probe resistance expression, Eq. (3.15), with the resistances of multi-probe conductors [12] further helped to clarify this equation.

Consider a four-probe conductor next. Let probe $k$ be the carrier source and probe $l$ be the carrier sink. Probes $m$ and $n$ are voltage probes. The voltmeter connected to a probe is taken to have infinite impedance. Hence, the voltage measured at a probe is obtained by adjusting the chemical potential of this probe such that the net current through the voltage probe is zero. To find the measured resistance in such a configuration, we have to solve Eq. (3.11) with the conditions $I \equiv I_{k} \equiv-I_{l}$ and $I_{m}=I_{n}=0$. We cannot directly invert Eq. (3.11) to find the chemical potentials as a function of the current. If all chemical potentials are equal, all the resulting currents are zero. Hence, the determinant of the matrix of transport coefficients in Eq. (3.11) is zero. A simple way to proceed is to subtract from each chemical potential in Eq. (3.11) the potential $\mu_{n}$. Writing the equations for $I_{k}, I_{l}$, and $I_{m}$ gives a system of three equations with three unknown variables, $\mu_{i}-\mu_{n}, i=k, l, m$. Solving for $\mu_{m}-\mu_{n}$ yield

$$
\mu_{m}-\mu_{n}=\frac{h}{e} I \frac{\left(T_{m k} T_{n l}-T_{m l} T_{n k}\right)}{D_{k n}} .
$$

Here, $D_{k n}$ is a subdeterminant of the matrix of transport coefficients in Eq. (3.12) of rank 3 (with row $k$ and column $n$ deleted from the full matrix). All subdeterminants of rank 3 of the matrix of transport coefficients are equal, $D_{k n} \equiv D$. This is a consequence of current conservation. To show this, let us denote the determinant of the full matrix by "det". It is zero. Now, expanding it in terms of subdeterminants yields

$$
0=\operatorname{det}=\left(M_{m}-R_{m m}\right) D_{m: n}-\sum_{n \neq m} T_{m n} D_{m n} .
$$

This is just Eq. (3.11) with zero current in all the probes and with the subdeterminants in place of the chemical potentials; but the only solution of Eq. (3.11) that yields zero current in all the probes is the equilibrium solution consisting of identical chemical potentials. Hence, all subdeterminants are equal, $D_{m n} \equiv D$. With the vo'tage 
drop, $V_{m n}=\left(\mu_{m}-\mu_{n}\right) / e$ as determined by Eq. (3.16), we find for the four-terminal resistance, $R_{k l, m n}=V_{m n} / I[12]$,

$$
R_{k l, m n}=\left(\frac{h}{e^{2}}\right) \frac{\left(T_{m k} T_{n l}-T_{m l} T_{n k}\right)}{D},
$$

Eq. (3.18) has the property that the two resistances measured in two configurations in which the current source and current sink are exchanged (exchange of $k$ and $l$ ) are equal in magnitude but differ in their sign. Similarly, two configurations that differ only in the voltage probes (exchange of $m$ and $n$ ) are equal up to a sign. The important symmetry of the four-terminal resistance, Eq. (3.18) is the reciprocity relation which states the following. The two resistances measured in the four-terminal configurations in which exchange of the role of the current and voltage probes is accompanied by a reversal of the magnetic field, are identical. The reciprocity of resistances,

$$
R_{k l, m n}(B)=R_{m n, k l}(-B)
$$

follows from Eq. (3.18) on account of the microreversibility property of the transmission probabilities, Eq. (3.7), and the invariance of the subdeterminant $D$ under field reversal. $D(\boldsymbol{B})=D(-\boldsymbol{B})$ follows from the fact that the subdeterminants also obey a reciprocity relation, $D_{m n}(B)=D_{n m}(-B)$, and that all subdeterminants are equal.

The key result of this chapter is Eq. (3.18) and in all our subsequent calculations, (3.18) will be used to find the transport properties of a system. The first step of this whole calculation procedure is that to obtain all the transmission and reflection probabilities, which is achieved by a finite element numerical method for solving the Schrödinger equation. 


\section{4}

\section{EFfeCt of an Antidot Potential}

\subsection{Introduction}

In Chapter 2, we have shown that whether it is the quantum Hall effect or the Aharonov-Bohm effect which appears in a particular sample (see § 2.6) depends on the relative magnitude of magnetic field $B$ and "constriction length" $W_{\mathfrak{a}}$ of that sample (Fig. (2.8)). If $B e W_{a}^{2} \ll 1$, Aharonov-Bohm oscillation will be the major effect, and on the other hand, if $B e W_{a}^{2} \gg 1$, quantum Hall effect will be manifested. It is the subject of this chapter to study the electronic transport properties in the crossover regime, i.e., $B e W_{a}^{2} \sim 1$.

A new and interesting development in the fabrication of antidot systems is due to Feng et. al.[67]. A multilevel fabrication technique allowed electric contact with the antidot, so that the size of the antidot could be varied by a potential. This experimental technique, in some sense, gives a controlled way to introduce artificial impurities at known positions inside a nanostructure, and thus opens many possibilities for systematically measuring conductance anomalies resulting from different arrangements of the artificial impurities. Among these, very interesting measurements were made on the whole range of magnetic fields where transport characteristics change from classical to quantum[68]. It was found, again, that coupling of the magnetic edge states[68] determines certain magneto-transport features, especially beating and sharp period changes in the resonant oscillations of the conductance.

Motivated by the experimental capability for controlling the size of an antidot $[67$, 68], in this chapter we present a theoretical study of the effects of antidot size on the magneto-resistance of a two-dimensional Hall junction. It is well known[4] that in mesoscopic physics many novel transport phenomena are related to the particular 
scattering potential landscape which confines the electron trajectory, and so we expect and indeed find several new features in various magneto-transport properties as the size of the antidot is changed. We focus on the formation and coupling of the magnetic edge states in this open system (see below), and classify quantum transport characteristics in several regimes as the antidot size is increased.

\subsection{Conductor with Ideal Propagation Channels}

Before studying the case where an antidot potential is present, let us now discuss how the quantum Hall effect is established in an open conductor with current probes in a Hall junction [22, 69, 24]. Fig. (4.1) shows a conductor where the Hall junction has twice the width as the probes connected to the current source and sink. Using the Büttiker's theory (see Chapter 3), edge states will be established along the samplc boundary. In the conductor of Fig. (4.1), $N$ edge states connect the four probes in a cyclical fashion. We assume that all the probes are ideal; i.e., the probes populate all edge states equally up to the chemical potential of the source. Furthermorc, since all probes are taken to be icieal, carriers that reach a probe leave the sample with probability of 1 . We further assume that the conductor is so wide that scattering across the sample from one edge to the other does not occur. Under these circumstances, the edge states provide perfect transmission probabilities, $T_{2 \mathrm{i}}=N, T_{32}=N, T_{43}=N$ and $T_{14}=N$. All the other transmission probabilities are zero. The reflection probabilities are determined by current conservation. If probe $i$ is described by a (large) number of states $M_{i}$ characterizing the metallic probe, current conservation requires $R_{i i}=M_{i}-N$. With the transmission and reflection probabilities specified it is casy to calculate the four-terminal resistance from Büttiker formula (Eq. (3.18)),

$$
R_{m n, k l}=\left(\frac{h}{e^{2}}\right) \frac{\left(T_{k m} T_{l n}-T_{k n} T_{l m}\right)}{D},
$$

where $T_{m n}$ is the transmission coefficient from probe $n$ to probe $m$, and the factor $D$ is a subdeterminant of rank three of the matrix formed by the equations for the electric current (see $\S 3.4$ ). For our structure (Fig. (4.1)), the Hall resistance is given by

$$
R_{13,24}=\left(\frac{h}{e^{2}}\right) \frac{\left(T_{21} T_{43}-T_{23} T_{41}\right)}{D}
$$




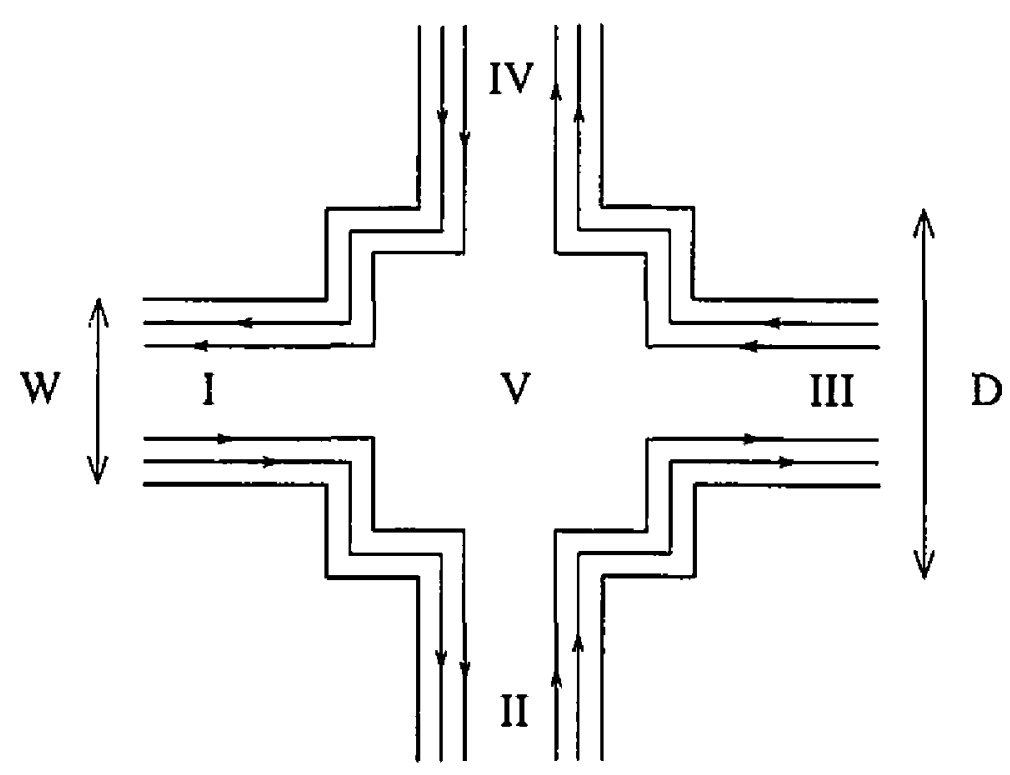

Figure 4.1: Conduetor with Hall probes and perfect edge states.

$R_{13,24}$ is determined by $T_{21} T_{43}-T_{23} T_{41}$, which is equal to $N^{2}$. Evaluation of the subdeterminant $D$ in Eq. (4.1) yields $D=N^{3}$. So, all Hall resistances of the conductor of Fig. (4.1) are quantized and given by

$$
R_{H}=\left(\frac{h}{e^{2}}\right)\left(\frac{1}{N}\right) .
$$

On the other hand longitudinal resistances, for example $R_{12,34}$, are zero. This is because in the products forming the expression for $R_{12,34}, T_{41} T_{23}-T_{43} T_{31}$, at least one transmission probability is always zero.

In the previous discussion, we assumed probes without internal reflection and assumed that backscattering is completely absent, i.e., the edge states provide perfect transmission channels for the carriers. These assumptions obviously are sufficient to obtain the quantum Hall effect. What the Hall resistance $R_{H}$ will be if these assumptions are no longer valid? To find out, we next consider the case when an antidot potential is present in the middle of the Hall junction. 


\subsection{Behavior of the Hall Resistance}

We study the ballistic structure shown in Fig. (4.2). It consists of an antidot confined inside a square quantum dot, with four probes connected to the quantum dot forming a Hall junction. As mentioned before, experimentally this system may now be fabricated using the multilevel fabrication technique[67, 70]. Without the probes this system is a chaotic billiard (the Sinai Billiard), and the motion of a classical particle inside it is chaotic[71]. With very narrow probes this structure may give rise to chaotic scattering of charge carries[72], thus it provides an interesting test ground for ideas and theories of the intriguing field of "quantum chaos"[71]. The study of "quantum chaos" are made by calculating the energy spectrum of this system, but it is not the subject of this thesis. What we study here is the physics between quantum Hall effect and Aharonov-Bohm effect.

In the present work we study situations where the probe width, $W$, is reasonably wide compared with the quantum dot size, $D$ (see Fig. (4.2)), thus we need not consider the possibility of chaotic scattering[73]. In this chapter, we fix the antidot in the center of the Hall junction and it is assumed to be circular with a radius $r_{a}$ which we shall vary. The whole system, including the probes, is penetrated by $a$ uniform external magnetic field $B$. A full quantum mechanical calculation of the electron scattering problem is very complicated even without the antidot[74] because of the irregular shape of the Hall junction, and thus theoretical investigations[24, 25] on junction scattering are usually based on physically motivated phenomenological models where such parameters as the coupling constants between different states are introduced. Here we solve the quantum scattering problem using a finite-element numerical method[28, 29] which allows us to clearly and directly demonstrate the formation and study the effects of magnetic edge states, and to obtain transport characteristics.

Throughout this calculation, we have fixed the dimensions of the structure to be $W=1650 \AA$, and $D=2 W$. We consider electrons coming from probe $\mathrm{I}$, which scatter inside the junction, and then reflect back or transmit to other probes. The confining potential at the boundaries of the structure, including the antidot, is assumed to be infinity. The single electron Schrödinger equation with effective mass approximation is 


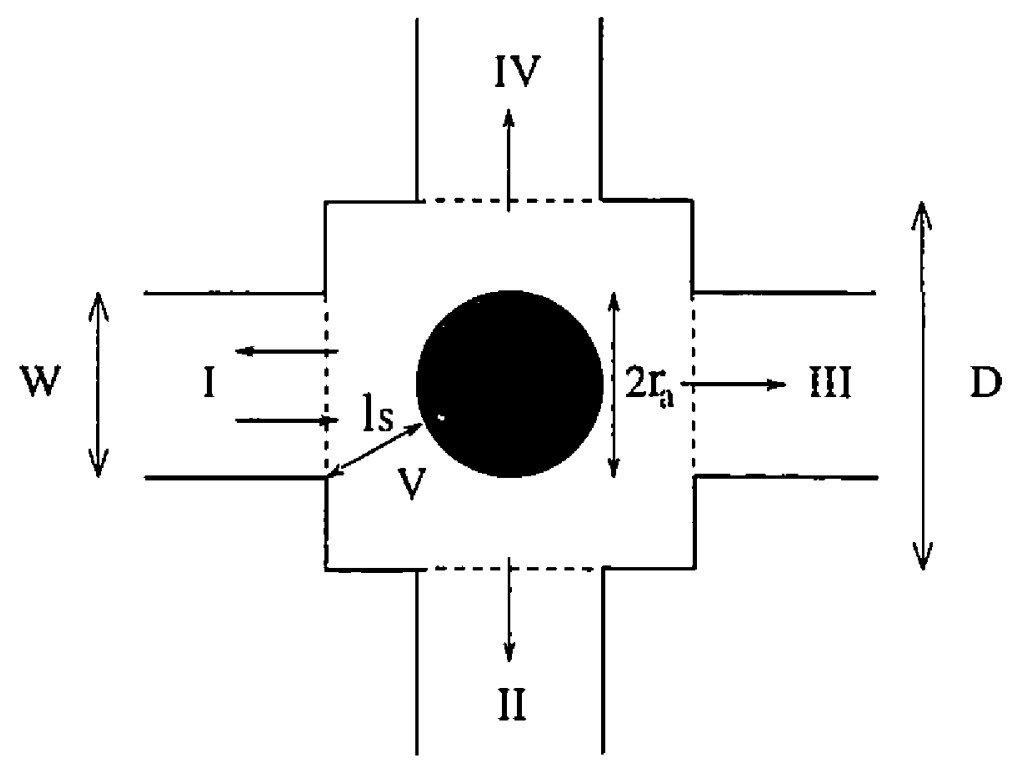

Figure 4.2: Schematic plot of the Hall junction. An antidot of radius $r_{a}$ is confined inside the square quantum dot. Electrons incident from probe I.

solved using the finite-element numerical scheme of Ref. [29]. Essentially we discretize the scattering region (the quantion dot) into a linear algebra problem, particularly a fine grid of finite elements which reduces the problem of solving the Schrödinger equation into a sparse matrix problem. The quantum propagation in the probes is solved separately using the technique outlined in Ref. [75]. The wave functions and their derivatives are then matched at the probe boundaries and this leads to the transmission coefficients $T_{n 1}$ ( $\left.n=1,2,3,4\right)$. We discretize the scattering region using 5000 grid points and check by increasing grid points further that excellent convergence is obtained. We note that extremely accurate solutions of the Schrödinger equation must be found for the probes especially at large values of $B$, otherwise accurate results of transmission cannot be obtained. As $r_{a}$ is varied, we make sure that at least two propagating modes are possible inside the junction even for the largest value of $r_{a}$. We fix the incoming electron energy by its momentum $k W=9.5$ which is just above the third subband when the magnetic field is absent. However, since the third subband cannot propagate in the probes when $B$ is larger than $\sim 1000$ Gauss, 
we only consider the first two subbands in our calculations. Finally, the Bütiker formula $[24,27] \mathrm{Eq} .(4.1)$ is used to compute various four-probe resistances. We need not calculate all other $T_{m n}$ 's $(n \neq 1)$ in Eq. (4.I), since our structure is four-fold symmetric. Thus the following relations will automatically hold for this structure:

$$
\begin{aligned}
& T_{11}=T_{22}=T_{33}=T_{44}, \\
& T_{21}=T_{32}=T_{43}=T_{14}, \\
& T_{41}=T_{34}=T_{23}=T_{12}, \\
& T_{31}=T_{13}=T_{24}=T_{42} .
\end{aligned}
$$

With these, the Hall resistance is given by

$$
R_{H}=R_{13,24}=\frac{h}{e^{2}} \frac{\left(T_{21} T_{21}-T_{41} T_{11}\right)}{D} .
$$

Generally speaking, Hall resistance is a function of both magnetic field $B$ and antidot radius $r_{a}$, i.e., $R_{H}=R_{H}\left(B, r_{a}\right)$. In this section, we pay morc attention to the relation between $R_{H}$ and $B$ and in next section we will inspect the dependence on $r_{a}$.

Fig. (4.3) shows Hall resistance $R_{H}$ as a function of magnetic field $B$, for several different antidot sizes $r_{a}$. Very different $R_{H}$ 's are clearly obtained for $r_{a}=0$ (no antidot, Fig. (4.3a)), small $r_{a}\left(r_{a}=0.25 W\right.$, Fig. (4.3b)), intermediate $r_{a}\left(r_{a}=0.5 W\right.$, Fig. (4.3c)), and large $r_{a}\left(r_{a}=0.75 W\right.$, Fig. (4.3d)). We will discuss them separately in the following.

When there is no artificial impurity, at low magnetic field $B$ the transmission is essentially dominated by quantum interference resulting in very complicated resistance fluctuations. We found that the first Hall plateau $(N=2)$ is reached when the magnetic field is such that the classical cyclotron radius equals $W / 2$, i.c. when the probes can exactly fit with a cyclotron orbit and all classical orbits originating from probe I can reach probe II. For our system this happens at around $B=4380$ Gauss, see Fig. (4.3a). Above this field strength the usual integer quantum Hall effect takes place[22], namely a further $R_{H}$ plateau is reached each time a propagating channel ceases to operate. In our case the second mode cannot propagate in the probes after $B=7180$ Gauss, and thus the second $R_{H}$ plateau is reached at this value of $B$. The 
4: Effect of an Antidot Potential
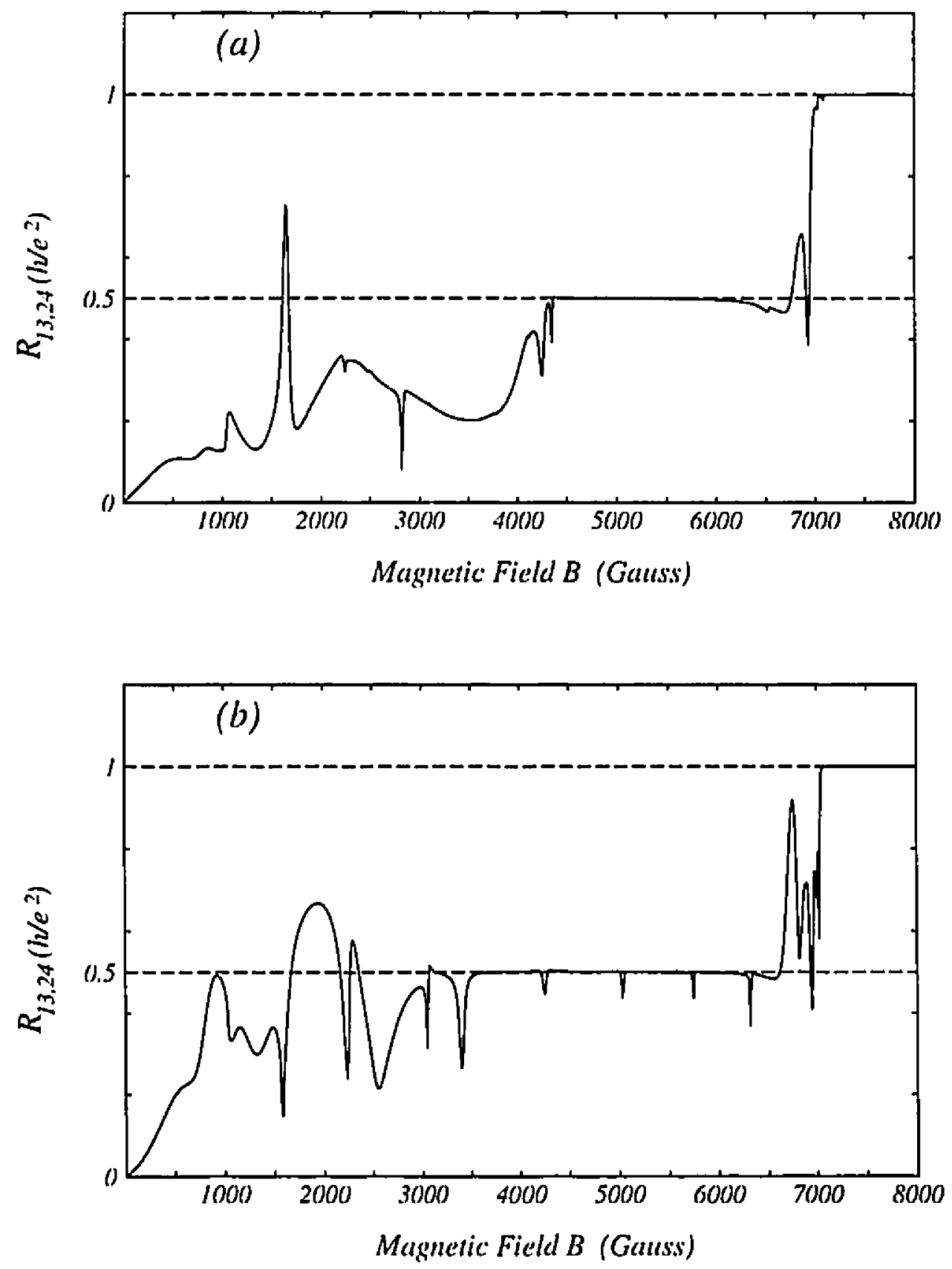

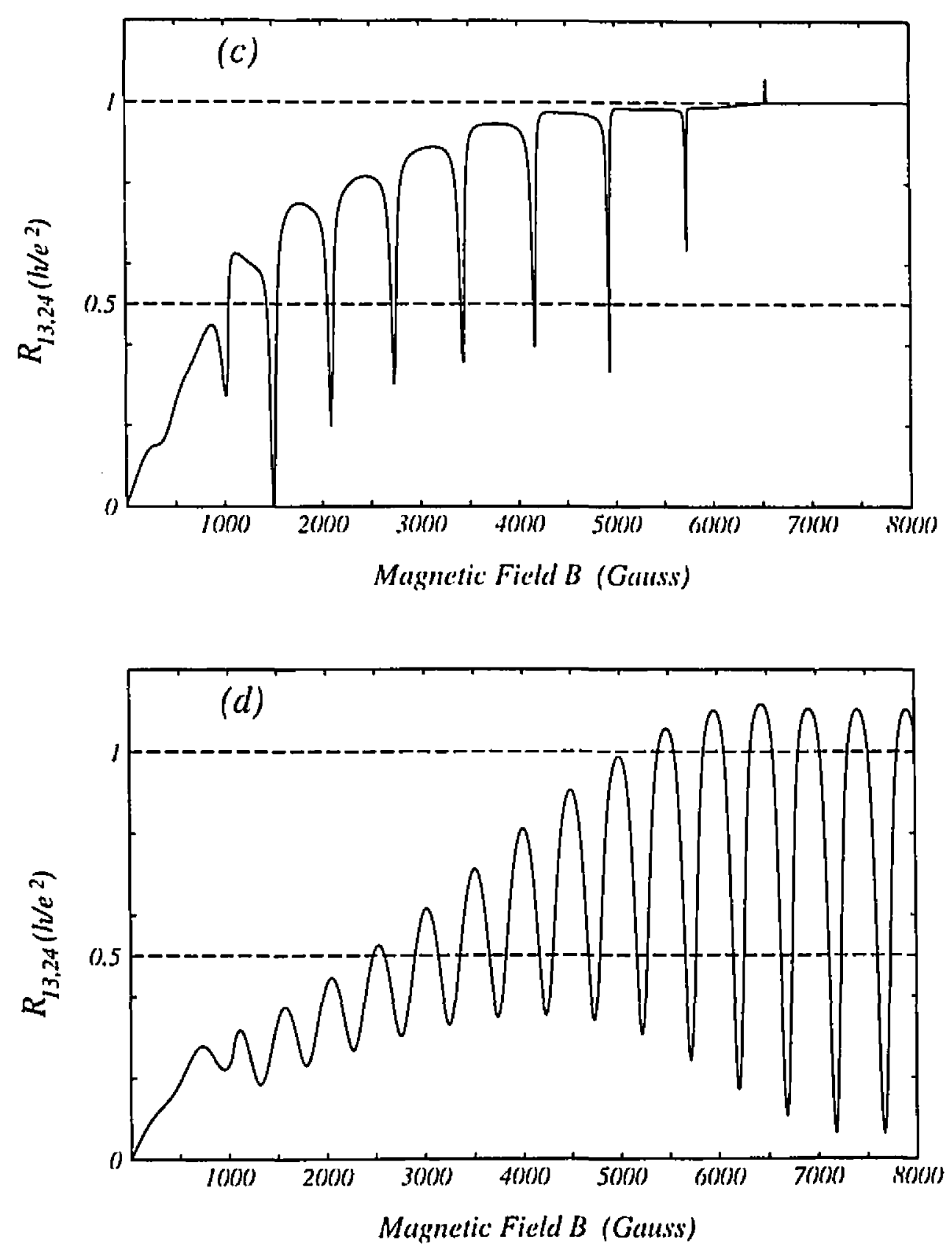

Figure 4.3: Hall resistance $R_{H}$ as a function of magnetic ficld $B$ for different antidot sizes $r_{a}$. (u) For $r_{a}=0$, integer quantum Hall regime is reached when perfectly transmitting edge states are established. (b) For $r_{a}=W / 4$. Coupling of the transmitting states to the localized states leads to the dips in the Hall plateau. (c) For $r_{a}=W / 2$ which is in the crossover regime where quantum Hall and $A B$ effects compete. (d) For $r_{a}=3 W / 4$. The Hall junction behaves as a ring for large $r_{a}$ and the transport is dominated by the Aharonov-Bohm oscillations. 
behavior of $R_{H}$ at low magnetic field is very complicated that is determined entirely by quantum interference. From an inspection of wave function, we found that the dips in Fig (4.3a), at low magnetic field correspond to localized states. From Fig. (4.3a), it is also casy to find that there is no sharp dips (i.e., localized states) once the quantum Hall regime has been reached and we shall demonstrate later in this chapter that this results from no antidot potential in the middle.

For a small antidot, such as $r_{a}=W / 4$, we can see from Fig. (4.3b) that the behavior of $R_{H}$ is essentially the same as that of case $r_{a}=0$. However the antidot can easily support localized edge states which "skip" around the antidot. The coupling of the localized edge state and the extended ones leads to the resonant-like sharp dips in the Hall plateau, as shown in Fig. (4.3b). Coupling to the localized edge state is clearly seen when we plot the norm of the wave function, $|\Psi(x, y)|^{2}$, at the dips of $R_{H}$, as shown in Fig. $(4.4 \mathrm{a}, 4.4 \mathrm{~b})$ at $B=6320$ Gauss. It turns out that for this structure and at this value of $B$ two localized edge states can be supported around the antidot, as shown by the two "rings" of the wave function. The outer state couples to the transmitting channels since it is closer to them, and causes the near periodic dips in the first Hall plateau. Similar resonance patterns are also observed in the quasi-one dimensional situation studied in the Ref. [23]. On the other hand, Figs. (4.4c, 4.4d) show $|\Psi(x, y)|^{2}$ on the plateau rather than at a dip, and clearly there is no coupling to the localized edge states. The antidot potential plays a crucial role for $R_{H}$ entering quantum Hall regime: scattering with antidot is helpful for electrons to transmit into probe II much before the classical cyclotron radius reaches the value $W / 2$. This can be clearly seen by comparing Fig. (4.3a) and (4.3b).

The above transport characteristics change qualitatively when the antidot size is further increased. At very large sizes of $r_{a}$, the structure behaves essentially like a quasi-one dimensional ring since the corners of the confining quantum dot will not be probed very much by the electrons. We found that this is indeed the case when the shortest length $l$, of the structure (see Fig. (4.2), which is the shortest distance from the surface of the antidot to the corner formed by the probe and the quantum dot, $l$, plays a role as the constriction length) is less than twice the magnetic length $l_{B}=\sqrt{\hbar c / e B}$. For this antidot size $\left(r_{a}=0.75 W\right)$, we have $l_{a}=588 \AA$. Thus if this, 


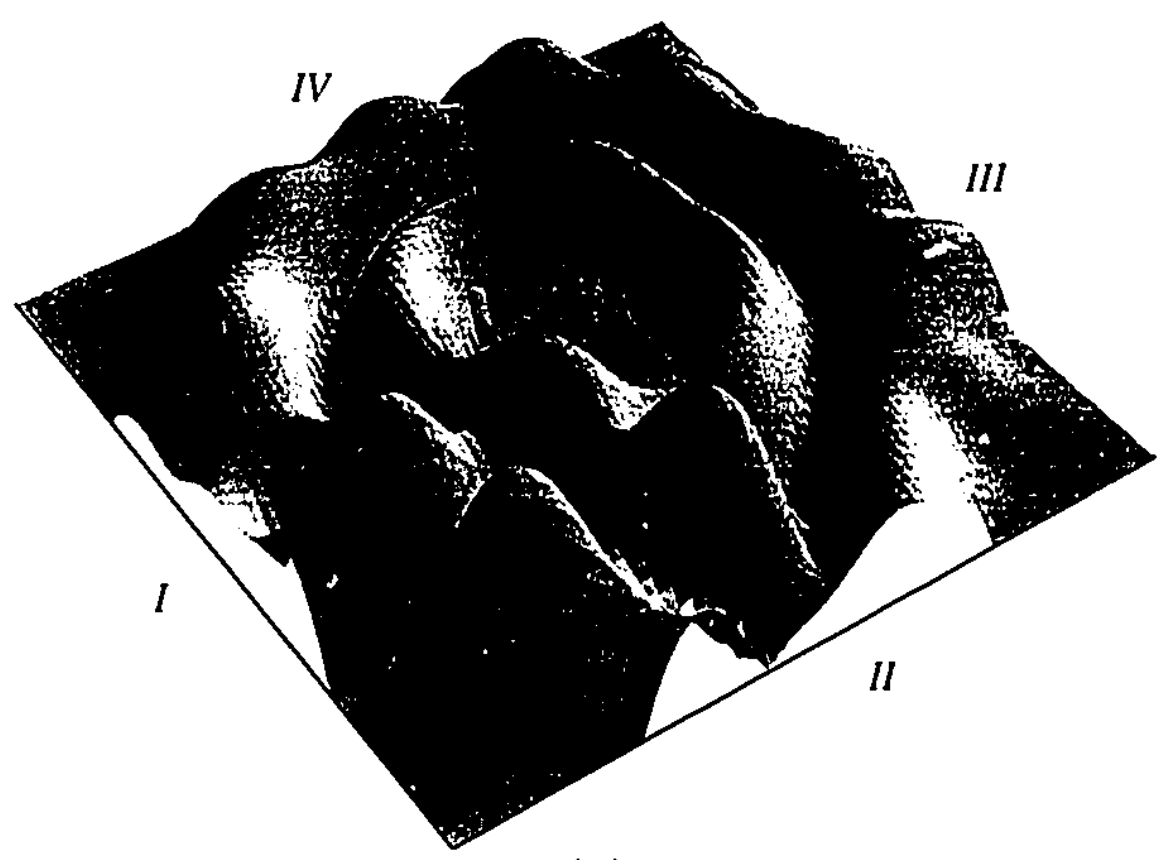

(a)

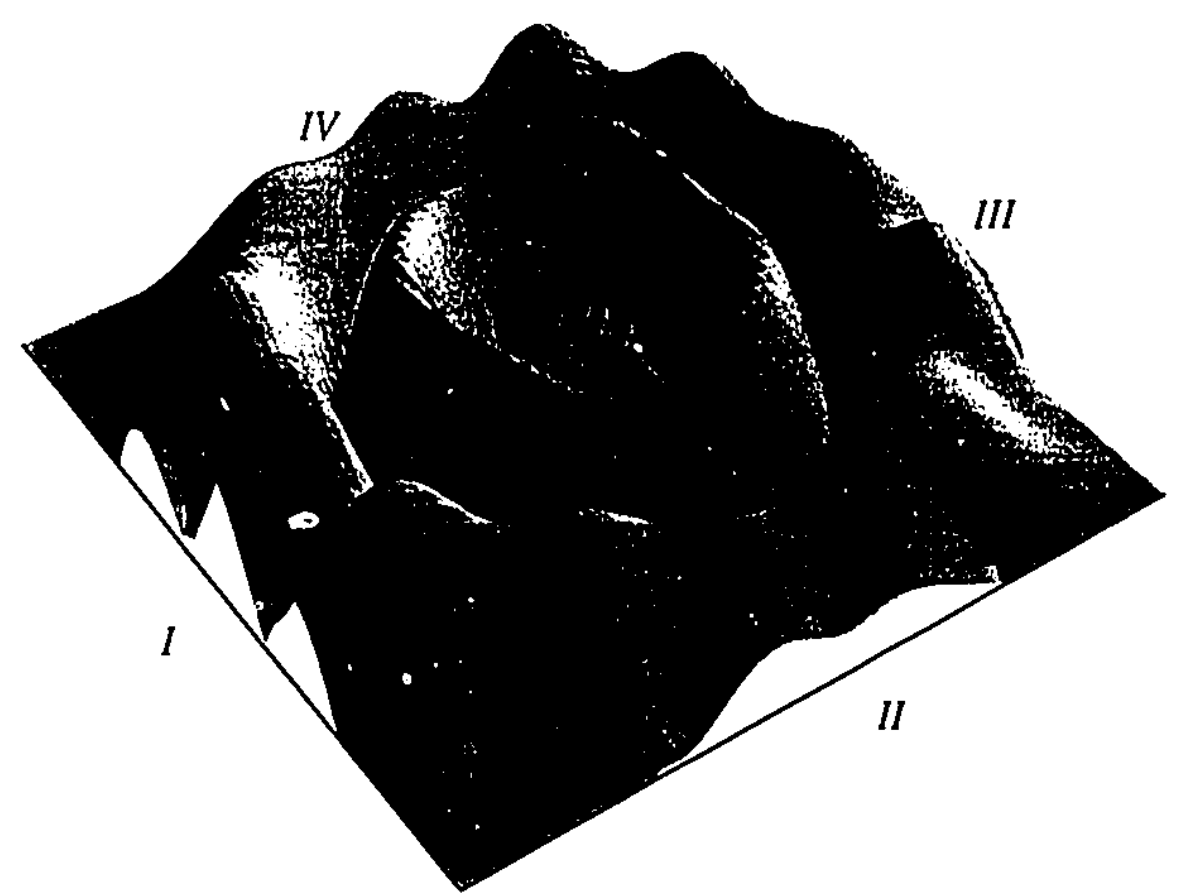

(b) 


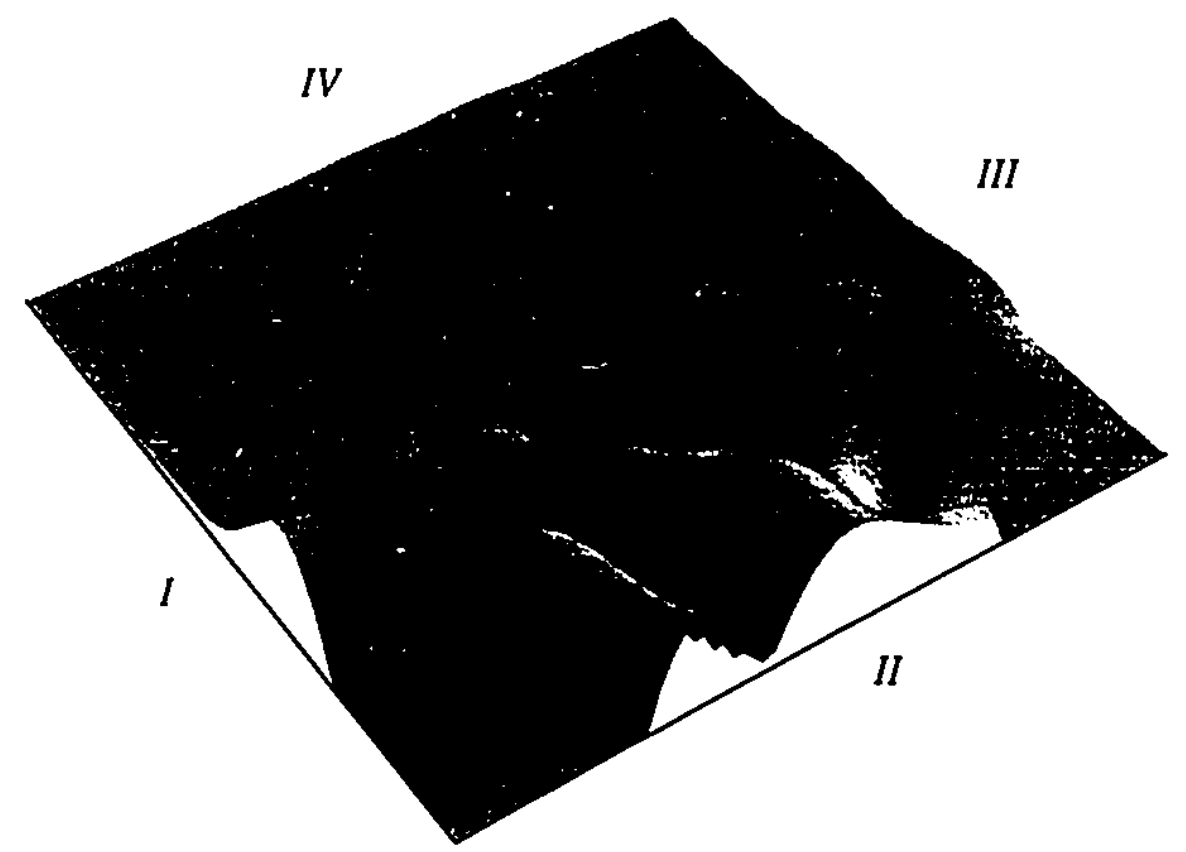

(c)

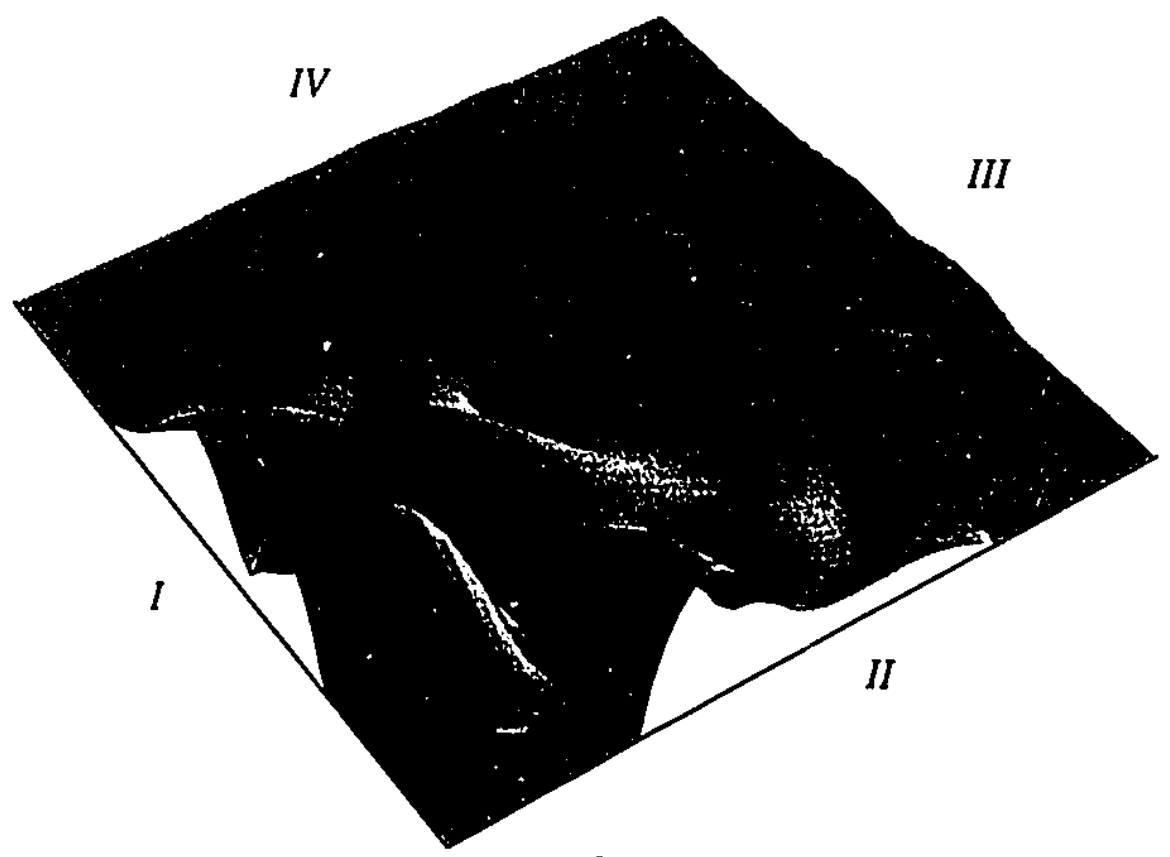

(d)

Figure 4.4: The norm $|\Psi(x, y)|^{2}$ for $r_{a}=W / 4$ in the quantum Hall regime: (a) and (b) At $B=6320$ Gauss where coupling to the localized state leads to a dip in the Hall plateau ((a) is for channel $1,(b)$ is for channel 2). Two localized states are clearly seen surrounding the antidot; (c) (d) At $B=6050$ Gauss where no coupling is established and the norm is predominately located around the path from probe I to probe II indicating the perfectly transmitting edge state ((c) is for channel 1 , (d) is for channel 2). Hall resistance at this $B$ takes the quantized value. 
$l_{s}<2 l_{B}$, the magnetic field $B$ must be less than 1.526 Tesla. Since the maximum value for $B$ in our calculation is 8000 Gauss, the structure with $r_{a}=0.75 W$ can indeed be thought as a quasi-one dimensional structure. Thus for large $r_{a}$ we expect to see typical $A B$ effect oscillations. Fig. (4.3d) shows this regime where almost perfect $A B$ oscillations are observed with a period given precisely by the size of the effective ring. For this antidot size, the effective ring radius is $W$. From the $\mathrm{AB}$ relation,

$$
S B=n \phi_{o}
$$

where $S$ is the ring area, $B$ the magnetic field, $\phi_{0}=h c / e$ the flux quantum, and $n$ an integer. Indeed, the period of the numerical data of Fig. (4.3d) is precisely given by $\phi_{0}$. Fig. (4.5) shows the norm of the wave functions for two different magnetic field. It is obvious that the radius of maximum-probability circle does not depends on the magnetic field $B$.

The most interesting regime as $r_{a}$ is varied is in between the quantum Hall and $A B$ effect regimes. When $r_{a}$ is not small enough to easily support formation of extended current carrying edge states (Fig. (4.3b)), yet not large enough to cause perfect $A B$ oscillation (Fig. (4.3d)), there is a range of intermediate values of $r_{a}$ where the Hall resistance behaves as shown in Fig. (4.3c). Here, for small magnetic field $B$, well established edge states are not formed and the transport is dominated by AB effect. This is shown as the oscillations in $R_{H}$ with rounded maxima, see Fig. (4.3c). The period of the oscillation increases as $B$ is increased, because a larger $B$ pushes the electron closer to the antidot, so that the quantum path surrounding the antidot becomes smaller, leading to a larger oscillation period. At higher magnetic fields, the quantum Hall regime is reached with a flat $R_{H}$ maximum and sharp dips reminiscent of Fig. (4.3b). The critical magnetic field separating these two behaviors is such that

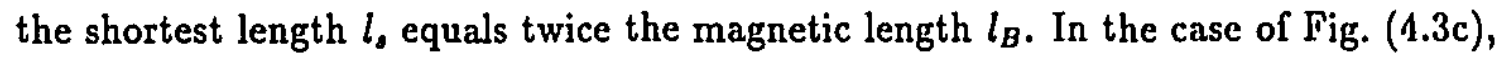
this value is about 2600 Gauss. Indeed we can see from Fig. (4.3c) that the Hall Resistance has a qualitative change in shape around this magnetic field value. The first Hall plateau (with $N=2$ ) disappears in Fig. (4.3c), a result of the AB oscillation. Although the true quantum Hall regime is reached only after $B=7180$ Gauss when the second propagating channel can rot transmit but the first channel is perfectly transmitting, we found that $R_{H}=e^{2} / h$ much before this value. In other words, there 
(a)

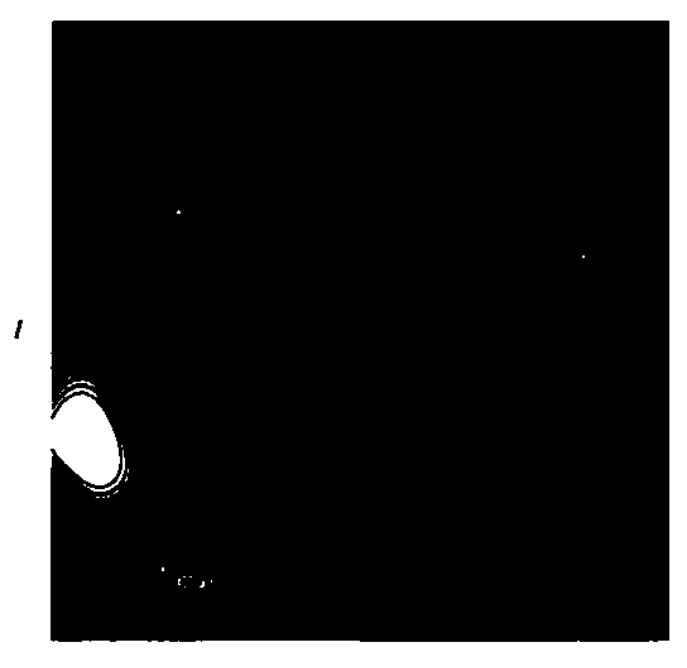

II

(c)

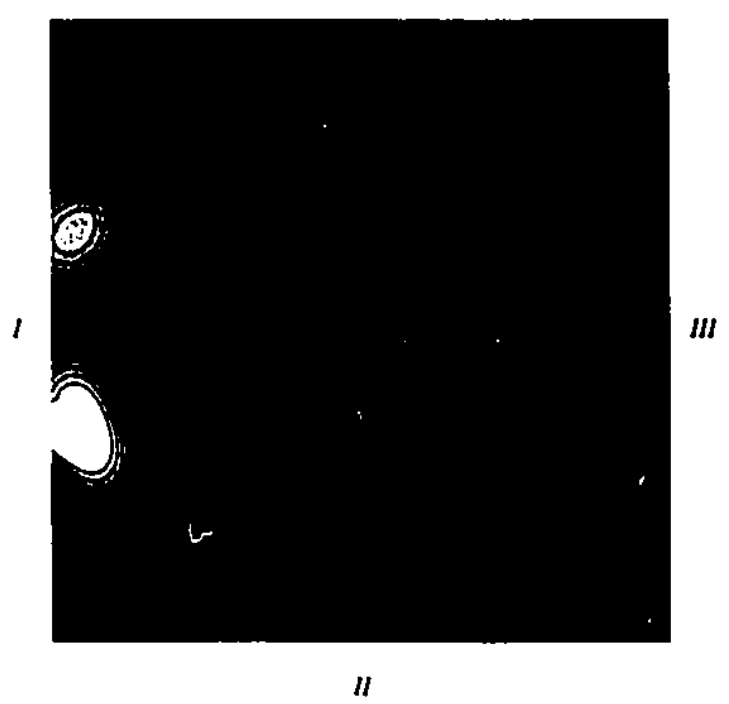

(b)

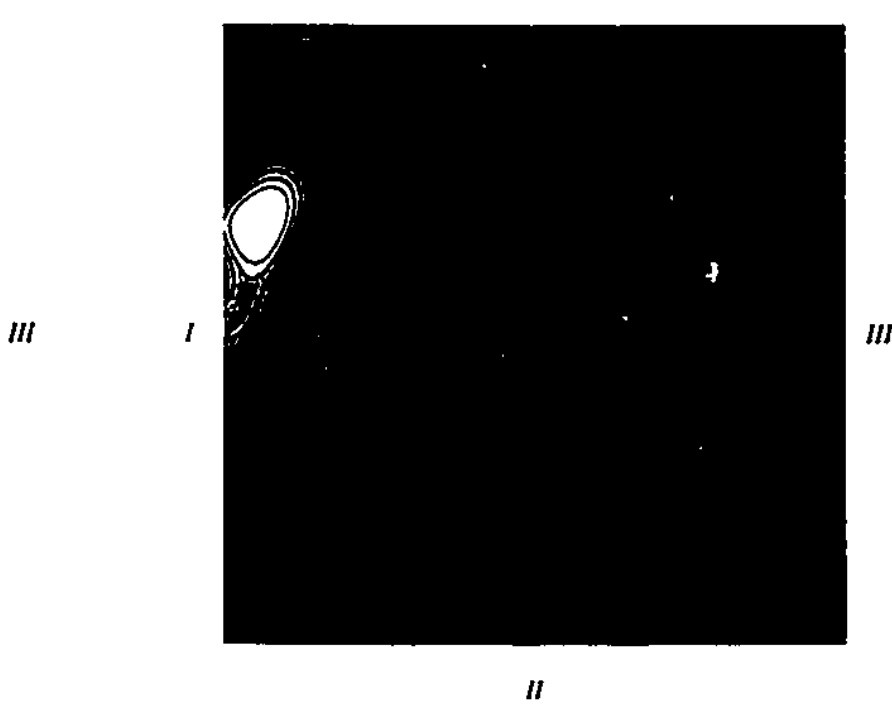

(d)

IV

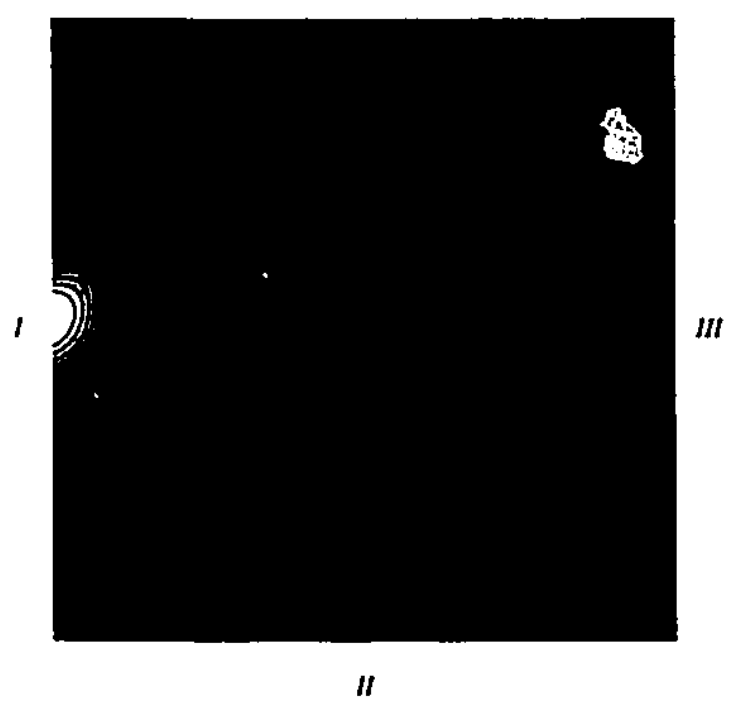

Figure 4.5: The Contour of norm of wave function for $r_{a}=3 W / 4$ at two different magnetic fields: (a) and (b) at $B=3304$ Gauss with incoming carrier in channel 1 and 2 respectively; (c) and (d) at $B=6450$ Gauss with incoming carrier in channel 1 and 2 respectively. Note the radius for maximum-probability circle is almost the same for the two different magnetic fields. 
is a range of magnetic field where both incoming channels are partially transmitting, but where there is an integer $(N=1)$ Hall resistance. We also found this to be the case for a range of antidot sizes, and in the next section we will show our calculation results of properties of $R_{H}$ as a function of $r_{a}$ at a fixed magnetic field.

The above results give a very rich behavior of the Hall resistance as the antidot size is increased. Essentially for the magnetic field range studied here, three transport regimes are observed: with small $r_{a}$ there is a well defined quanium Hall regime; at large $r_{a} \mathrm{AB}$ oscillations dominate transport; and at intermediate values of $r_{a}$ there is a crossover regime. In the next section we examine this last transport regime in more detail, to reveal some peculiar properties.

\subsection{Crossover Regime}

As discussed in section 4.3, a peculiar property of the crossover regime is that apparently a Hall plateau is obtained when the two incoming modes are both partially transmitting. Similar behavior was observed in Ref. [75] in a cross-Hall-bar without any artificial impurity, but no explanation was given. Obviously this phenomenon is completely different from the so called "last plateau" problem[76], since it only happens in the crossover regime. In the particular system we studied $\left(r_{a}=0.5 W\right)$ ), $R_{H}$ takes the value $1 \times \frac{h}{e^{2}}$ for a quite large range of magnetic field (about 3500 Gauss, we call it "crossover regime of $B$ ") before $B$ reaches 7180 Gauss, the value at which the second incoming channel becomes evanescent while the first channel becomes perfectly transmitting. There turns out to exist an approximate symmetry between transmission coefficients of individual incoming channels which leads $R_{H}$ to take this quantized value before the quantum Hall regime is reached. The phenomenon occurs for a range of the antidot size $r_{a}$, and in Fig. (4.6a) and Fig. (4.6b) we plot various transmission coefficients and Hall resistance respectively as a function of $r_{a}$ for a fixed magnetic field value $B=5450$ Gauss, which belongs to the "crossover regime of $B$ ". As shown in Fig. (4.6), when $r_{\alpha}$ is small, at this magnetic field there are two perfectly transmitting modes hence $T_{21}=N=2$ while $T_{41}=T_{31}=T_{11}=0$, where $T_{m n}$ is the total transmission coefficients from probe $n$ to probe $m$ including all propagating modes. This gives the Hall plateau $R_{H}=\frac{1}{2} h / e^{2}$, as discussed in the section 4.2. But 

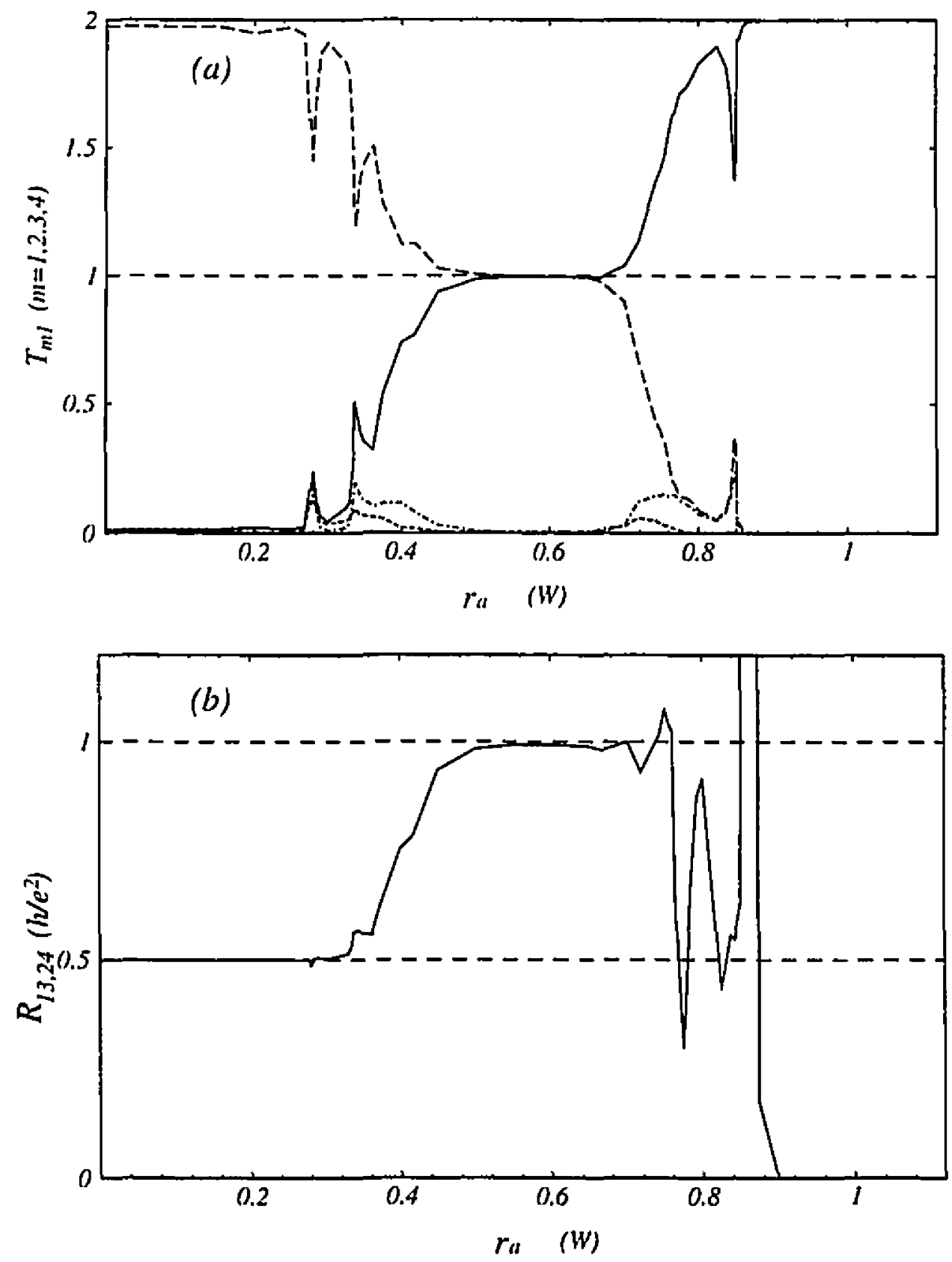

Figure 4.6: (a) Transmission coefficients $T_{m n}$ as a function of the antidot size $r_{a}$ at $B=5450$ Gauss, where subscript $m n$ indicating transmission from probe $n$ to probe $m$. Note in the crossover regime $T_{11}=T_{21}=1$ while $T_{31}=T_{41}=0$. Solid line is $T_{11}$, dashed line is $T_{21}$, short-dashed line is $T_{31}$ and dot-dashed line is $T_{41}$. (b) Hall resistance $R_{H}$ as a function of the antidot size $r_{a}$ at $B=5450$ Gauss. It is obvious that there is a crossover regime where Hall resistance $R_{H}$ takes a abnormal integer value of $h / e^{2}, i . e, N=1$ 


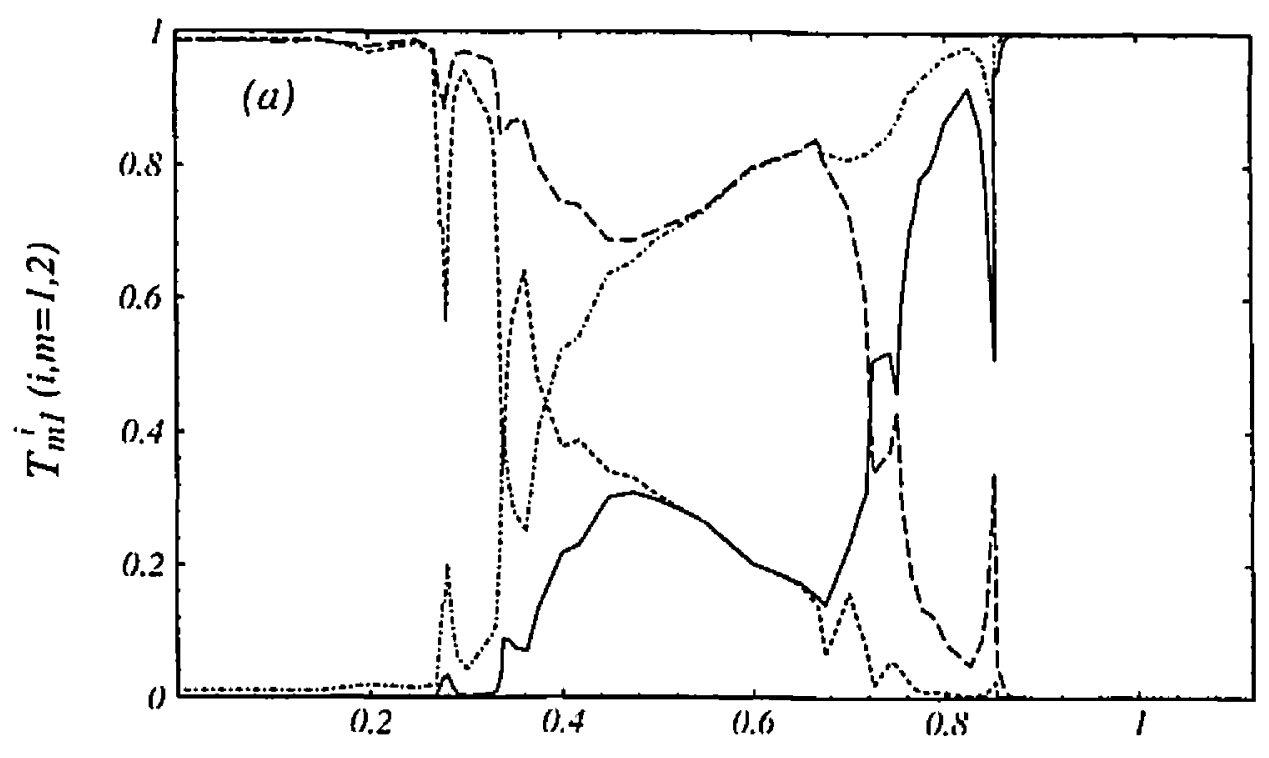

ras (W)

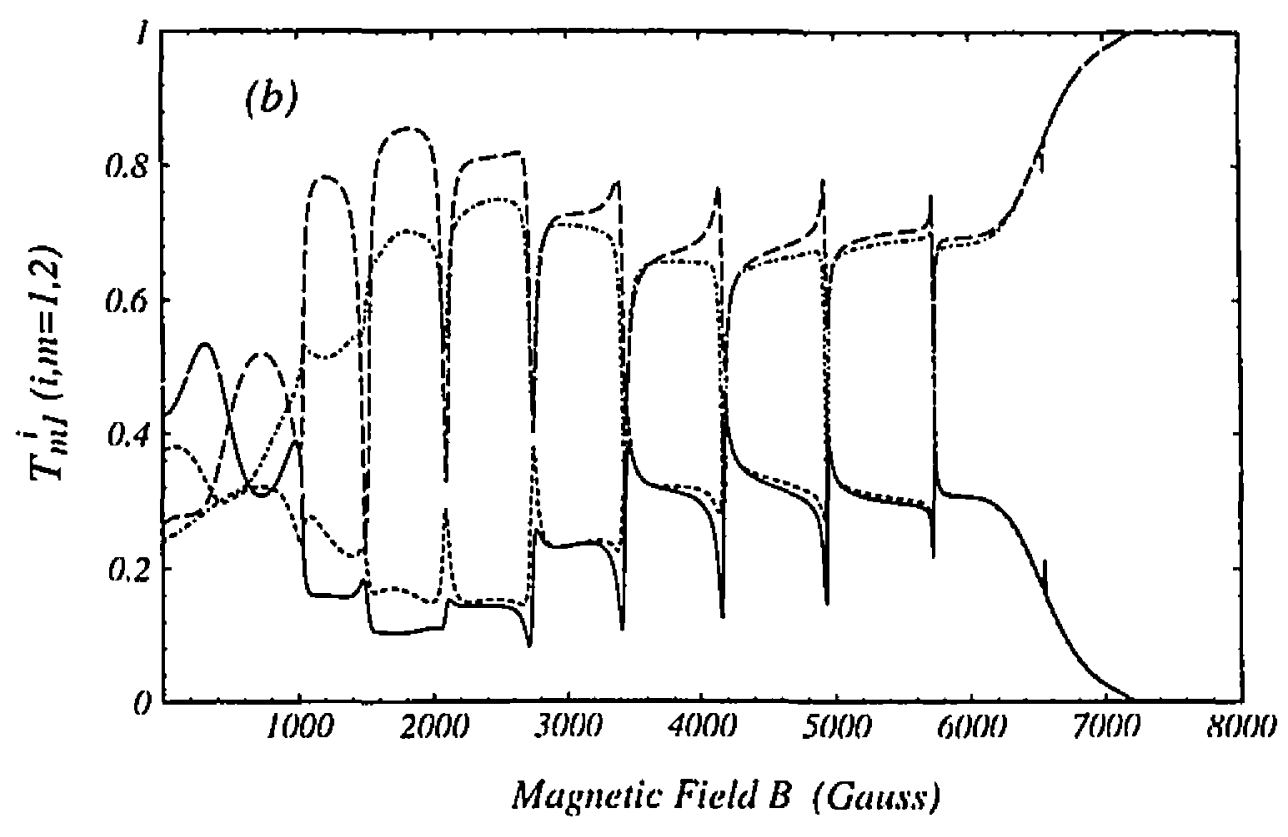

Figure 4.7: (a) Transmission coefficients of individual incoming channels $T_{m n}^{i}$, where $i$ is the channel number, as a function of $r_{a}$ at $B=5450$ Gauss. Note in the crossover regime $T_{11}^{1}=T_{21}^{2}, T_{21}^{1}=T_{11}^{2}$. (b) Transmission coefficients of individual incoming channels $T_{m n}^{i}$ as a function of magnetic field $B$ at $r_{a}=W / 2$ which gives clear crossover regime. Notice at higher $B$ a peculiar symmetry exists: $T_{11}^{1}=T_{21}^{2}$ and $T_{21}^{1}=T_{11}^{2}$. In (a) and (b), solid line is $T_{11}^{1}$, dashed line is $T_{21}^{1}$, short-dashed line is $T_{21}^{2}$ and dot-dashed line is $T_{11}^{2}$. 
at intermediate values of $r_{a}$, i.e. in the crossover regime when $0.50 \mathrm{~W}<r_{\mathrm{a}}<0.70 \mathrm{~W}$, Fig. (4.6a) shows that $T_{11}=T_{21}=1$ while $T_{31}=T_{41}=0$, and these lead to the abnormal plateau.

Because there are two incoming channels contributing to these values, Fig. (4.7) shows the transmission coefficients for the individual channels. Inspecting Fig. (4.7) in the crossover regime, the following relations are seen to hold:

$$
\begin{aligned}
& T_{11}^{1}\left(B, r_{a}\right)+T_{11}^{2}\left(B, r_{a}\right)=T_{11}\left(B, r_{a}\right)=1 \\
& T_{21}^{1}\left(B, r_{a}\right)+T_{21}^{2}\left(B, r_{a}\right)=T_{21}\left(B, r_{a}\right)=1, \\
& T_{31}^{1}\left(B, r_{a}\right)+T_{31}^{2}\left(B, r_{a}\right)=T_{31}\left(B, r_{a}\right)=0, \\
& T_{41}^{1}\left(B, r_{a}\right)+T_{41}^{2}\left(B, r_{a}\right)=T_{41}\left(B, r_{a}\right)=0,
\end{aligned}
$$

where $T_{m n}^{i}\left(B, r_{a}\right)$ is the transmission coefficient from probe $n$ to probe $m$ when incoming electron is in channel $i$. For simplicity we omit $B$ and $r_{a}$ and just use $T_{m n}^{i}$

\begin{tabular}{|l|ccccc|}
\hline \hline & \multicolumn{5}{|c|}{$r_{a}=0.6 \mathrm{~W}$} \\
$T_{i j, m n}$ & $B=5204 G$ & $B=5446 G$ & $B=5688 G$ & $B=5930 G$ & $B=6172 G$ \\
\hline$T_{11,11}$ & 0.03863 & 0.03242 & 0.04029 & 0.03236 & 0.02503 \\
$T_{22,21}$ & 0.04376 & 0.03203 & 0.05163 & 0.03682 & 0.02429 \\
\hline$T_{22,11}$ & 0.58821 & 0.62563 & 0.57451 & 0.62607 & 0.68944 \\
$T_{11,21}$ & 0.58225 & 0.61535 & 0.58326 & 0.63434 & 0.70191 \\
\hline$T_{21,11}$ & 0.18579 & 0.16969 & 0.19330 & 0.17205 & 0.14322 \\
$T_{12,21}$ & 0.17963 & 0.16924 & 0.17853 & 0.16467 & 0.14239 \\
\hline$T_{12,11}$ & 0.18434 & 0.16805 & 0.19173 & 0.17051 & 0.14203 \\
$\boldsymbol{T}_{2 \mathrm{i}, 21}$ & 0.19253 & 0.18184 & 0.18246 & 0.16165 & 0.12967 \\
\hline
\end{tabular}

Table 4.1: Transmission coefficients $T_{i j, m n}$, which is the probability of an electron incoming from probe $n$ at channel $j$ and going into probe $m$ at channel $i$, as a function of magnetic field at $r_{a}=0.6 \mathrm{~W}$. The data shows approximately the "micro-symmetry" of Eqs. (4.36).

to represent $T_{m n}^{i}\left(B, r_{a}\right)$ later on. Furthermore, our data of Fig. (4.7) shows another 


\begin{tabular}{|l|cccc|}
\hline \hline & \multicolumn{4}{|c|}{$B=6172$ Gauss } \\
$T_{i j, m n}$ & $r_{a}=0.50 \mathrm{~W}$ & $r_{\mathrm{a}}=0.55 \mathrm{~W}$ & $r_{\mathrm{a}}=0.60 \mathrm{~W}$ & $r_{\mathrm{a}}=0.65 \mathrm{~W}$ \\
\hline$T_{11,11}$ & 0.06948 & 0.04364 & 0.02503 & 0.02421 \\
$T_{22,21}$ & 0.08273 & 0.04385 & 0.02429 & 0.01124 \\
\hline$T_{22,11}$ & 0.47411 & 0.58414 & 0.68944 & 0.77547 \\
$T_{11,21}$ & 0.47757 & 0.59881 & 0.70191 & 0.77431 \\
\hline$T_{21,11}$ & 0.22729 & 0.18791 & 0.14322 & 0.10185 \\
$T_{12,21}$ & 0.21284 & 0.17963 & 0.14239 & 0.10994 \\
\hline$T_{12,11}$ & 0.22266 & 0.18434 & 0.14203 & 0.10156 \\
$T_{21,21}$ & 0.22669 & 0.18791 & 0.12967 & 0.09764 \\
\hline
\end{tabular}

Table 4.2: Transmission coefficients $T_{i j, m n}$ for different antidot size $r_{a}$ at $B=6172$ Gauss. The data shows approximately the "micro-symmetry" of Eqs. (4.36).

peculiar set of symmetry relations at the crossover regime (Since $T_{31}^{1}$ and $T_{11}^{1}$ are almost zero, we don't show them in Fig. (4.7).):

$$
T_{11}^{1}=T_{21}^{2}, T_{21}^{1}=T_{11}^{2}, T_{\dot{3}}^{\imath}=T_{41}^{2}, T_{41}^{1}=T_{31}^{2}
$$

which indicate that the reflection coefficient of channel $1, T_{11}^{1}$, equals the transmission coefficient to probe II of channel $2, T_{21}^{2}$; and that the transmission coefficient to probe III of channel 1, $T_{31}^{1}$, equals the transmission coefficient to probe IV of channel $2, T_{41}^{2}$. These symmetry relations are nontrivial. Because of them, according to Eq. (3.18) we obtain $R_{H}=1 \times \frac{h}{e^{2}}$ which is twice the value in the absence of the antidot. This is peculiar since transport is not in the quantized Hall regime as perfectly transmitting channels have not been formed. Finally, if we denote $T_{i j, m n}$ as the probability of an electron incoming from probe $n$ at channel $j$, and going into probe $m$ at channel $i$, then our calculation shows approximately the following "micro-symmetry",

$$
T_{11,11}=T_{22,21} \quad, \quad T_{22,11}=T_{11,21}
$$

as summarized in Tables (4.1) and (4.2). Although this "micro-symmetry" cannot be measured experimentally, it is most interesting because it obviously gives rise to the 
relations (4.7) and (4.8), and thus leads to the peculiar transport properties of the crossover regime.

\subsection{Topological Explanation of Crossover Regime}

Why do we have these special "micro-symmetry" properties? To answer this question 'we extend the scattering matrix approach [22] to multiprobe conductors. Before that, let us first generally investigate the electronic transport property of a fourprobe conductor (shown in Fig. (4.2)). From a semi-classical point of view, with reasonably large magnetic field $B$ we expect edge states to from inside the scattering junction. A particular scenario is sketched in Fig. (4.8). For a small antidot as shown in Fig. (4.8a), perfectly transmitting states can easily form, which may be coupled with a localized state "skipping" around the antidot. On the other hand for a large antidot, it is more difficult for an electron to enter the scattering region, and the perfectly reflecting states may couple with the localized state which "skips" along the walls of the quantum dot, as shown in Fig. (4.8b). We can also get the same two propagation patterns as above through a pure topological consideration. For the sake of discussion, suppose that only one quantum channel (edge state) in each probe is opened and electrons coming from probe I. Once the magnetic field is strong enough to form edge states, the scattering problem turns into a problem of finding out how the Hall junction scatters the carrier into the outgoing channel of the four probes. It is obvious that transmitting into probe II and reflecting back into probe I have much larger probability than exiting from the other two probes. If we connect the incoming channel with these two outgoing edge states and assume that carrier would leave scattering region through probe I and II with probability 1 respectively (provided no coupling between edge states and localized state), we shall obtain two transport patterns as shown in Fig. (4.8).

The localized state mediates interaction of one edge state of the conductor with another edge state on the other side of the conductor. The interaction of the localized state and edge states in the probes for patterns of Figs. (4.8a) and (4.8b) can be characterized by complex $3 \times 3$ scattering matrices $\boldsymbol{S}^{(a)}$ and $\boldsymbol{S}^{(b)}$, which are of the 

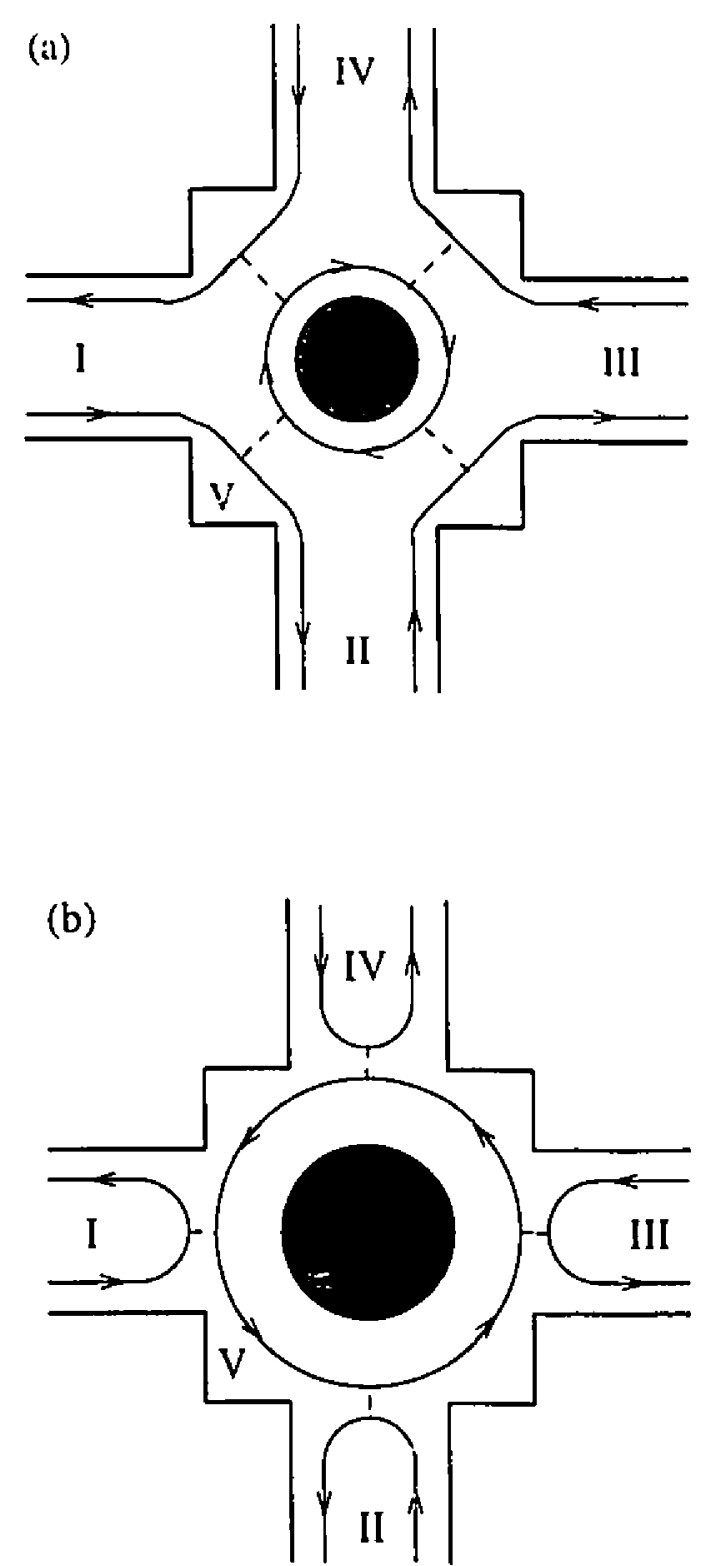

Figure 4.8: Schematic plot of the two important transmission patterns in the quantum Hall regirne. (a) For small antidot sizes. Perfectly transmitting edge states (solid lines) with possible coupling (broken line) to the localized state surrounding the antidot (the circulating line). (b) For large antidot sizes. Perfectly reflecting edge states with possible coupling to the localized state "skipping" along the walls of the quantum dot. The two transmission patterns are topologically equivalent by exchanging transmission to reflection probabilities. 
form[22]

$$
S^{(a)}=\left(\begin{array}{ccc}
t_{11}^{m} & t_{21}^{m} & t_{l 1}^{m} \\
t_{12}^{m} & t_{22}^{m} & t_{l 2}^{m} \\
t_{1 l}^{m} & t_{2 l}^{m} & r_{l}^{m}
\end{array}\right) \quad, \quad S^{(b)}=\left(\begin{array}{ccc}
r_{11}^{m} & r_{21}^{m} & t_{l 1}^{m} \\
r_{12}^{m} & r_{22}^{m} & t_{l 2}^{m} \\
t_{1 l}^{m} & t_{2 l}^{m} & r_{l}^{m}
\end{array}\right) \text {. }
$$

Here sattering amplitudes have the following meaning: $t_{i l}^{m}$ gives the probability of an electron transferring from the localized state labeled $l$ to reach the edge state in channel $i$ in probe $m$. $t_{l i}^{m}$ is the probability that an electron transfers from edge state $i$ in probe $m$ to the localized state $l$. Micro-reversibility implies that $t_{i l}^{m}=t_{l i}^{m}$. $l_{i j}^{m}$ of $S^{(a)}$ is the transmission probability that an electron incidents in probe $m$ in channel $j$ and exits in channel $i$ (here we assume that carriers only incident in probe I) while $r_{i j}^{m}$ of $S^{(b)}$ is the reflection probability. $r_{l}^{m}$ is the probability of a carrier in the localized state to continue stay at the localized state past the most probable tunneling path connecting it to probe $m$. For the symmetric system studied here, we will drop the superscript $m$ in the scattering amplitude to simplify notation without causing confusion. Probability (current) conservation requires

$$
\begin{aligned}
& \left|t_{11}\right|^{2}+\left|t_{21}\right|^{2}+\left|t_{l 1}\right|^{2}=1 \\
& \left|t_{12}\right|^{2}+\left|t_{22}\right|^{2}+\left|t_{l 2}\right|^{2}=1 \\
& \left|r_{11}\right|^{2}+\left|r_{21}\right|^{2}+\left|t_{l 1}\right|^{2}=1 \\
& \left|r_{12}\right|^{2}+\left|r_{22}\right|^{2}+\left|t_{l 2}\right|^{2}=1 \\
& \left|t_{1 l}\right|^{2}+\left|t_{2 l}\right|^{2}+\left|r_{l}\right|^{2}=1
\end{aligned}
$$

Following the topological equivalence argument of Büttiker[24], from a scattering point of view, the configurations in Fig. (4.8a) and (4.8b) are equivalent: in the ideal situations of Fig. (4.8) the transmission and reflection matrix of the transmission pattern shown in Fig. (4.8a) is obtained by a permutation of the transmission and reflection matrix of Fig. (4.8b), i.e. the role of the reflection probabilities in pattern Fig. (4.8b) is taken by the transmission probabilities of pattern Fig. (4.8a):

$$
\left(\begin{array}{ccc}
t_{11}^{(a)} & t_{21}^{(a)} & t_{l 1}^{(a)} \\
t_{12}^{(a)} & t_{22}^{(a)} & t_{l 2}^{(a)} \\
t_{1 l}^{(a)} & t_{2 l}^{(a)} & r_{l}^{(a)}
\end{array}\right) \Longleftrightarrow\left(\begin{array}{ccc}
r_{22}^{(b)} & r_{12}^{(b)} & t_{2 l}^{(b)} \\
r_{21}^{(b)} & r_{11}^{(b)} & t_{1 l}^{(b)} \\
t_{l 2}^{(b)} & t_{l 1}^{(b)} & r_{l}^{(b)}
\end{array}\right)
$$


Let us denote the phase that is accumulated by a carrier on the localized state during traversal from one possible escape path (a probe) to the next escape path. Let us calculate the transmission probabilities from one probe to another. Consider the complex amplitude $t_{11,11}^{(a)}$. This amplitude comes from the following processes. First, there is a direct transmission with probability,

$$
t_{1 l}^{(a)} e^{i \phi_{1}} t_{l 1}^{(a)}
$$

Second, there is the possibility that a carrier completes $n$ full turns on the locnlized state before escaping, and the amplitude for that is

$$
t_{11,11}^{(a)}=t_{1 l}^{(a)} e^{i \phi_{1}}\left[r_{l}^{i} e^{i\left(\phi_{1}+\phi_{2}+\phi_{3}+\phi_{4}\right)}\right]^{n} t_{l 1}^{(a)} .
$$

Summing over all these amplitudes, we obtain a total transmission amplitude,

$$
t_{11,11}^{(a)}=\frac{t_{11}^{(a)} e^{i \phi_{1}} t_{l 1}^{(a)}}{1-r_{l}^{4} e^{i\left(\phi_{1}+\phi_{2}+\phi_{3}+\phi_{4}\right)}} .
$$

Next, we parameterize the reflection coefficients in terms of their amplitudes and phases,

$$
r_{l}=\sqrt{R_{l}} e^{i \Delta \phi}
$$

This gives a transmission probability,

$$
T_{11,11}^{(a)}=\left|t_{11,11}^{(a)}\right|^{2}=\frac{T_{1 l}^{(a)} T_{l 1}^{(a)}}{1+R_{l}^{4}-2 R_{l}^{2} \cos (\chi)}
$$

where $T_{i j}^{(a)}=\left|t_{i j}^{(a)}\right|^{2},(i, j=1,2, l, i \neq j)$. with a total phase,

$$
\chi=\sum_{i=1}^{i=1}\left(\phi_{i}+\Delta \phi\right) \text {. }
$$

Similarly, we can get

$$
t_{22,21}^{(b)}=\frac{t_{2 l}^{(b)} e^{i \phi_{1}} t_{l 2}^{(b)}}{1-r_{l}^{i} e^{i\left(\phi_{1}+\phi_{2}+\phi_{3}+\phi_{4}\right)}}
$$

and

$$
T_{22,21}^{(b)}=\left|t_{22,21}^{(b)}\right|^{2}=\frac{T_{2 l}^{(b)} T_{l 2}^{(b)}}{1+R_{l}^{4}-2 R_{l}^{2} \cos (\chi)} .
$$

Making use of the permutation relation (4.16), we obtain

$$
t_{11,21}^{(a)}=t_{22,11}^{(b)}
$$


and

$$
T_{11,21}^{(a)}=T_{22,11}^{(b)}
$$

In light of the above procedure, it is straightforward to derive all the following symmetry relations for transmission patterns in Figs. (4.8a) and (4.8b):

$$
\begin{gathered}
T_{i j, 11}^{(a)}=T_{j i, 21}^{(b)}, \quad T_{i j, 21}^{(a)}=T_{j i, 11}^{(b)}, \quad T_{i j, 31}^{(a)}=T_{j i, 41}^{(b)}, \\
T_{i j, 41}^{(a)}=T_{j i, 31}^{(b)} \quad(i \neq j, i, j=1,2) \\
T_{i i, 11}^{(a)}=T_{j j, 21}^{(b)}, \quad T_{i i, 21}^{(a)}=T_{j j, 11}^{(b)}, \quad T_{i i, 31}^{(a)}=T_{j j, 41}^{(b)}, \\
T_{i i, 41}^{(a)}=T_{j j, 31}^{(b)} \quad(i \neq j, i, j=1,2)
\end{gathered}
$$

here the superscript $(a, b)$ indicates the transmission patterns of Fig. (4.8a) or $(4.8 \mathrm{~b})$. With these relations established, we can now investigate the size effect of the antidot.

In general when there is an antidot in the middle of the scattering region, both the first and second incoming channels can probably transport with patterns (a) and (b). When radius of the antidot is small enough, the two incoming channels almost both have transmission pattern of Fig. (4.8a). On the other hand, they will both propagate with pattern of Fig. (4.8b) if the antidot size is large. In the crossover regime, both transmission patterns are possible, thus in general we may extrapolate between the two propagating patterns. Assuming $T_{i j, m n}$ is a linear combination of $T_{i j, m n}^{(a)}$ and $T_{i j, m n}^{(b)}$, we find

$$
T_{i j, m n}=\alpha_{1}\left(r_{a}, B\right) T_{i j, m n}^{(a)}+\beta_{1}\left(r_{a}, B\right) T_{i j, m n}^{(b)}
$$

where $\alpha_{1}\left(r_{a}, B\right)$ and $\beta_{1}\left(r_{a}, B\right)$ are the probabilities that the first incoming channel propagates with pattern (a) and pattern (b) respectively; and $\alpha_{2}\left(r_{a}, B\right), \beta_{2}\left(r_{a}, B\right)$ are those of the second incoming channel. These quantities are functions of the antidot size $r_{a}$ and the magnetic field $B$. Probability conservation requires

$$
\begin{aligned}
& \alpha_{1}\left(r_{a}, B\right)+\beta_{1}\left(r_{a}, B\right)=1, \\
& \alpha_{2}\left(r_{a}, B\right)+\beta_{2}\left(r_{a}, B\right)=1 .
\end{aligned}
$$

Particularly, for $T_{11,11}$ we have

$$
T_{11,11}=\alpha_{1}\left(r_{a}, B\right) T_{11,11}^{(a)}+\beta_{1}\left(r_{a}, B\right) T_{11,11}^{(b)}
$$



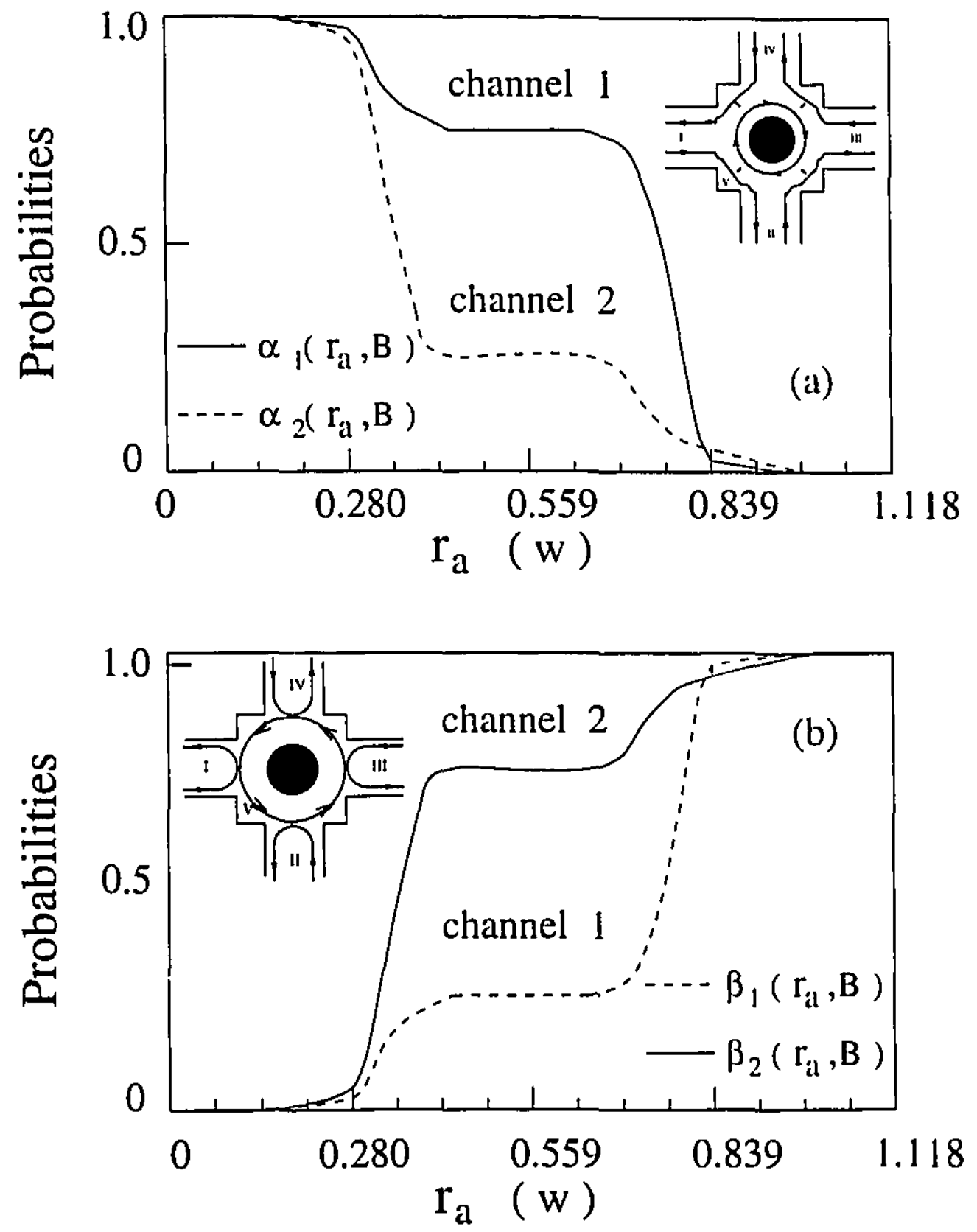

Figure 4.9: Schematic plot of the extrapolation parameters $\alpha_{i}\left(r_{a}, B\right)$ and $\beta_{i}\left(r_{a}, B\right)$ as extracted from the numerical data of Fig. (4.6a), where subscript $i$ indicates the incoming channel. Solid line: for channel 1; broken line: for channel 2. (a) For propagation pattern of Fig. (4.8a). (b) For propagation pattern of Fig. (4.8b). 
Making use of symmetry properties Eqs. (4.27), (4.28), we obtain

$$
T_{11,11}=\alpha_{1}\left(r_{a}, B\right) T_{11,11}^{(a)}+\beta_{1}\left(r_{a}, B\right) T_{22,21}^{(a)}
$$

We can also obtain

$$
T_{22,21}=\alpha_{2}\left(r_{a}, B\right) T_{22,21}^{(a)}+\beta_{2}\left(r_{a}, B\right) T_{11,11}^{(a)}
$$

Compare the above two relations, we find that if

$$
\alpha_{1}\left(r_{a}, B\right)=\beta_{2}\left(r_{a}, B\right)
$$

we then obtain the following symmetry property which is observed in our numerical data as discussed above, e.g. Eq. (4.9): $T_{11,11}=T_{22,21}$. Similarly a host of other "micro-symmetries" are also obtained for the crossover regime:

$$
\begin{gathered}
T_{22,11}=T_{11,21} \quad T_{21,11}=T_{12,21} \\
T_{12,11}=T_{21,21} \quad T_{11,31}=T_{22,41} \\
T_{22,31}=T_{11,41} \quad T_{21,31}=T_{12,41} \\
T_{12,31}=T_{21,41} .
\end{gathered}
$$

As discussed in $\S 4.3$, these symmetry relations lead to the peculiar transport properties of the crossover regime. These relations can be directly tested from our numerical data of Tables (4.1) and (4.2) and they are indeed quite well satisfied by the data for the range of the magnetic field and the antidot size corresponding to the crossover regime.

The scattering probabilities $\alpha_{i}\left(r_{a}, B\right), \beta_{i}\left(r_{a}, B\right)(i=1,2)$ can be extracted from our numerical data, and are shown in Fig. (4.9) for a fixed magnetic field $B$. Indeed, in the intermediate range of values of $r_{a}$ where the crossover regime lies, Eq. (4.35) is well satisfied. It is also easy to see the direct relation between Fig. (4.6) and Fig. (4.9).

\subsection{Effect of Resonant State on the Quantum Hall Effect}

In the last section, we have given a topological explanation for the "micro-symmetry" in the crossover regime. In Fig. (4.9), especially the reciprocal property in the 
crossover regime, is the base of our findings. Since we can not directly compute the values for pattern probabilities $\alpha$ and $\beta$ explicitly in our calculation, in this section we address the resonant behavior of the transport process analytically and make use of that to justify our assumption (Fig. (4.9)). Resonant tunneling processes requires phase coherence. Recent experiments in $G a A s$ structures $[77,78,79,15,40]$ suggest that the phase-coherence length is of the order of a few $\mu \mathrm{m}$ at low temperature. Here the structure we studied (Fig. (4.2)) has the dimension of $\sim 3 \mu \mathrm{m}$, so that phase-coherence requirement is satisfied. Our main interest is the effect of localized states on the Hall resistance $[80,81,82,83,24]$.

Consider now the four-terminal conductor in Fig. (4.8) and for simplicity we consider the case where only one edge state is present. As suggested alrendy, there are two major important transmission patterns in the quantum Hall regime (Fig. (4.8)), and the effect of scattering region is characterized by scattering matrices, $S^{(a)}$ and $\boldsymbol{S}^{(b)}$ respectively. Matrix elements $t_{i j}, t_{i l}, t_{l i}, r_{l}$, and $r_{i j}$ have the same meaning as those defined iefore (see $\S 4.5$ ).

Let us first investigate the transmission pattern shown in Fig. (4.8a). In § 4.5, we have derived the transmission probability $T_{11,11}^{(a)}$, Eq. (4.21). Since we have assumed that there is only one quantum channel available for carrier propagation, we have

$$
T_{11}^{(a)}=T_{11,21}^{(a)}=\frac{T_{1 l} T_{l 1}}{1+R_{l}^{4}-2 R_{l}^{2} \cos (\chi)},
$$

where the definitions of $\chi, R_{l}$ are the same as that in $\S 4.5$. With the same method discussed in $\S 4.4$, we can calculate other transmission probabilities. For the transmission probability $T_{31}$, we obtain

$$
T_{31}^{(\alpha)}=\frac{T_{1 l} T_{l 1} R_{l}^{2}}{1+R_{l}^{4}-2 R_{l}^{2} \cos (\chi)},
$$

and for $T_{41}$,

$$
T_{41}^{(a)}=\frac{T_{1 l} T_{l 1} R_{l}}{1+R_{l}^{4}-2 R_{l}^{2} \cos (\chi)} .
$$

The transmission probability $T_{21}$ is found by current conservation,

$$
T_{21}^{(a)}=1-T_{11}^{(a)}-T_{31}^{(a)}-T_{11}^{(a)} .
$$

In a similar fashion, we can determine all the other transmission and reflection probabilities. 


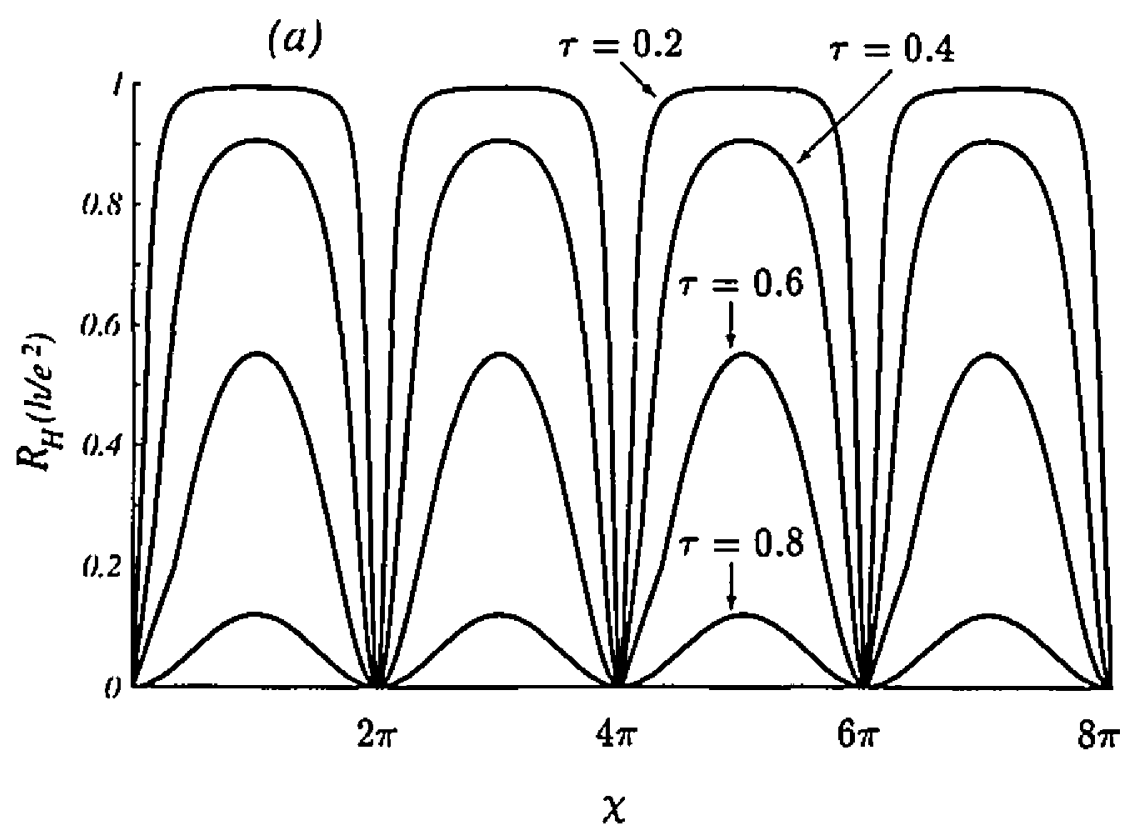

(b)

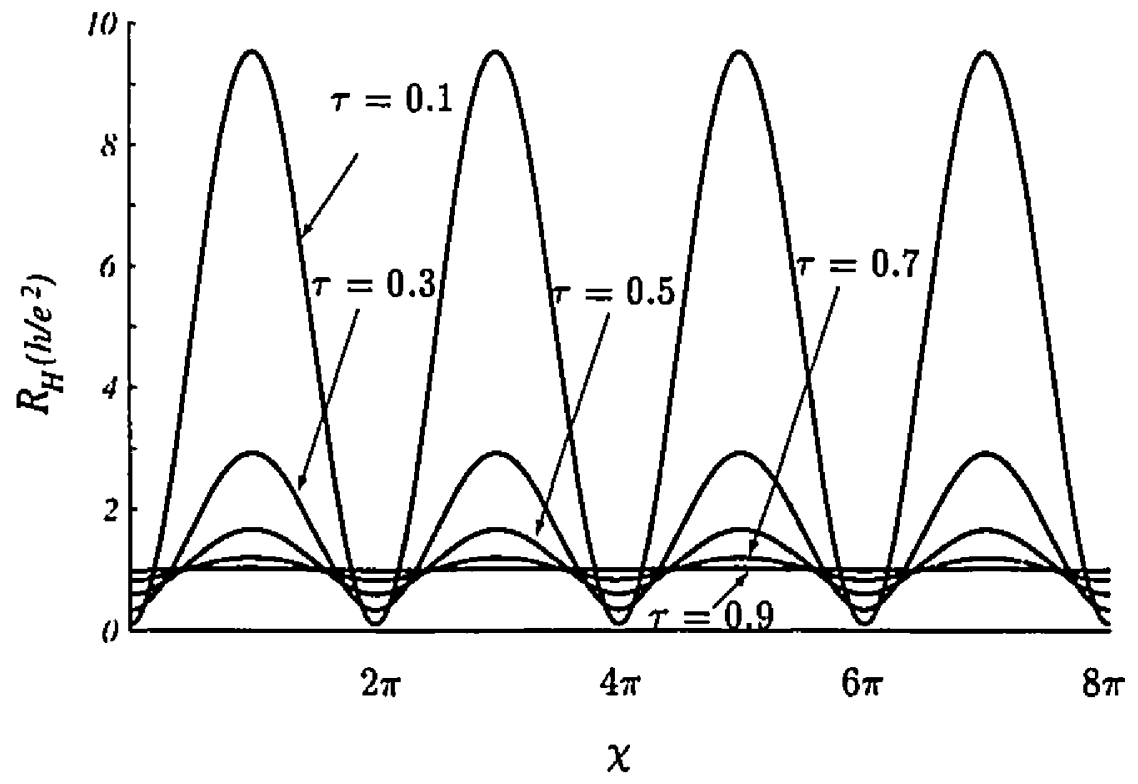

Figure 4.10: Hall resistance $R_{H}$ of a four-fold symmetric conductor with a resonant state in the center. (a) For transmission pattern as in Fig. (4.8a) with one edge state only. (b) For transmission pattern as in Fig. (4.8b) with one edge state only. In (a) and (b), $r$ is the transition probability between edge state and localized state. 
Here we focus on the case where all the probes are identical, i.e., on the case of a conductor that is fourfold-symmetric. The Hall resistance is calculated with the help of Eq. (4.1) and transmission probabilities as determined above. We find the Hall resistance

$$
R_{H}=\frac{h}{e^{2}} \frac{1-F\left(1+R_{l}\right)^{2}}{1-2 F R_{l}\left(1+R_{l}\right)+F^{2}\left(1+R_{l}\right)^{2}\left(1+R_{l}^{2}\right)}
$$

Where $F$ is of the form

$$
F=\frac{\tau^{2}}{1+R_{l}^{4}-2 R_{l}^{2} \cos (\chi)}
$$

with

$$
\tau=\sqrt{T_{1 l} T_{11}}=1-R_{l},
$$

which is the transition probability between edge state and localized state. Fig. (4.10a) shows the Hall resistance as a function of the total phase $\chi$ for different transition probability $\tau$. It is a periodic function of $\chi$. There is no interaction between the edge states and localized state if $\tau=0$. Even very small interactions (small transmission probabilities $T_{i l}$ and $T_{l i}$ ) lead to sharp deviations from the quantized value when $\chi=0$. Note that at these values of the phase, the Hall resistance is zero (completely quenched). With increasing transmission probabilities, the interaction between the edge states and the localized states increases and the Hall resistance for all $\lambda$ lies below the quantized value. Finally, if the interaction of the edge states and the localized states becomes strong $(\tau \approx 1)$, the Hall resistance becomes smail over the entire range of $\chi$.

Next, consider the conductor in Fig. (4.8b). The pattern of current carrying edge states at the Fermi energy of this conductor again can be mapped onto that of the conductor in Fig. (4.8a). The scattering matrix for the conductor of Fig. (4.8b) can be obtained by a permutation of the scattering matrix of the conductor in Fig. (4.8n) using "topological equivalence". In particular, for the transmission probabilities, we find

$$
T_{11}^{(a)}=T_{21}^{(b)}, T_{21}^{(a)}=T_{11}^{(b)}, T_{31}^{(a)}=T_{11}^{(b)}, T_{11}^{(a)}=T_{31}^{(b)}
$$

We can get the Hall resistance for this case to be

$$
R_{H}=\frac{h}{e^{2}} \frac{1}{\tau^{2}} \frac{\left(1-R_{l}^{2}\right)\left(1+R_{l}^{4}-2 R_{l}^{2} \cos (\chi)\right)}{\left(1+R_{l}^{2}\right)\left(1+R_{l}\right)^{2}} .
$$



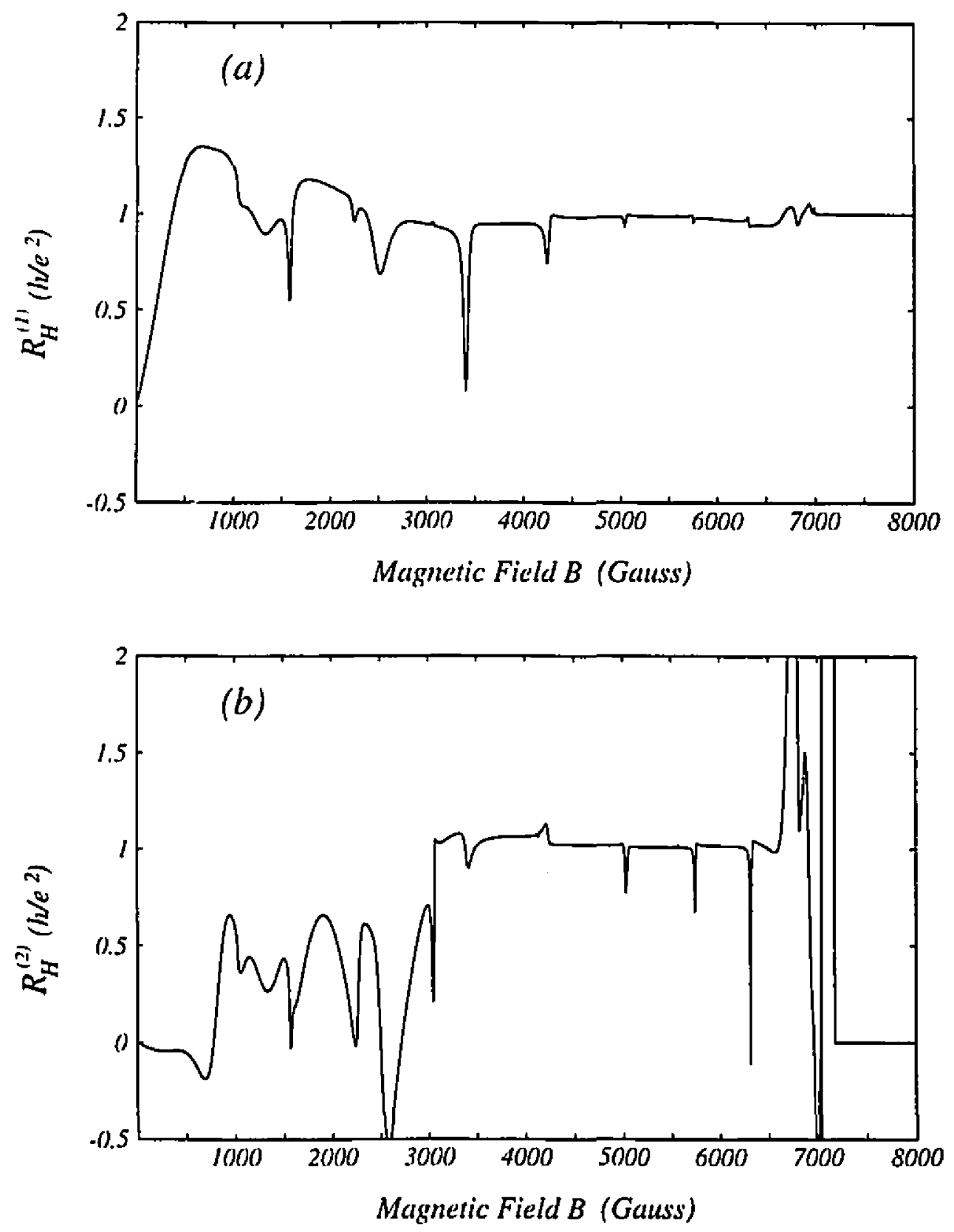

Figure 4.11: Hall resistance $R_{H}$ as a function of magnetic field $B$ for small antidot size $\left(r_{a}=0.25 W\right)$. ((a) is for channel 1 and (b) is for channel 2). The behavior of $R_{H}$ of both (a) and (b) are more like that of Fig. $(4.10 \mathrm{a})$. 

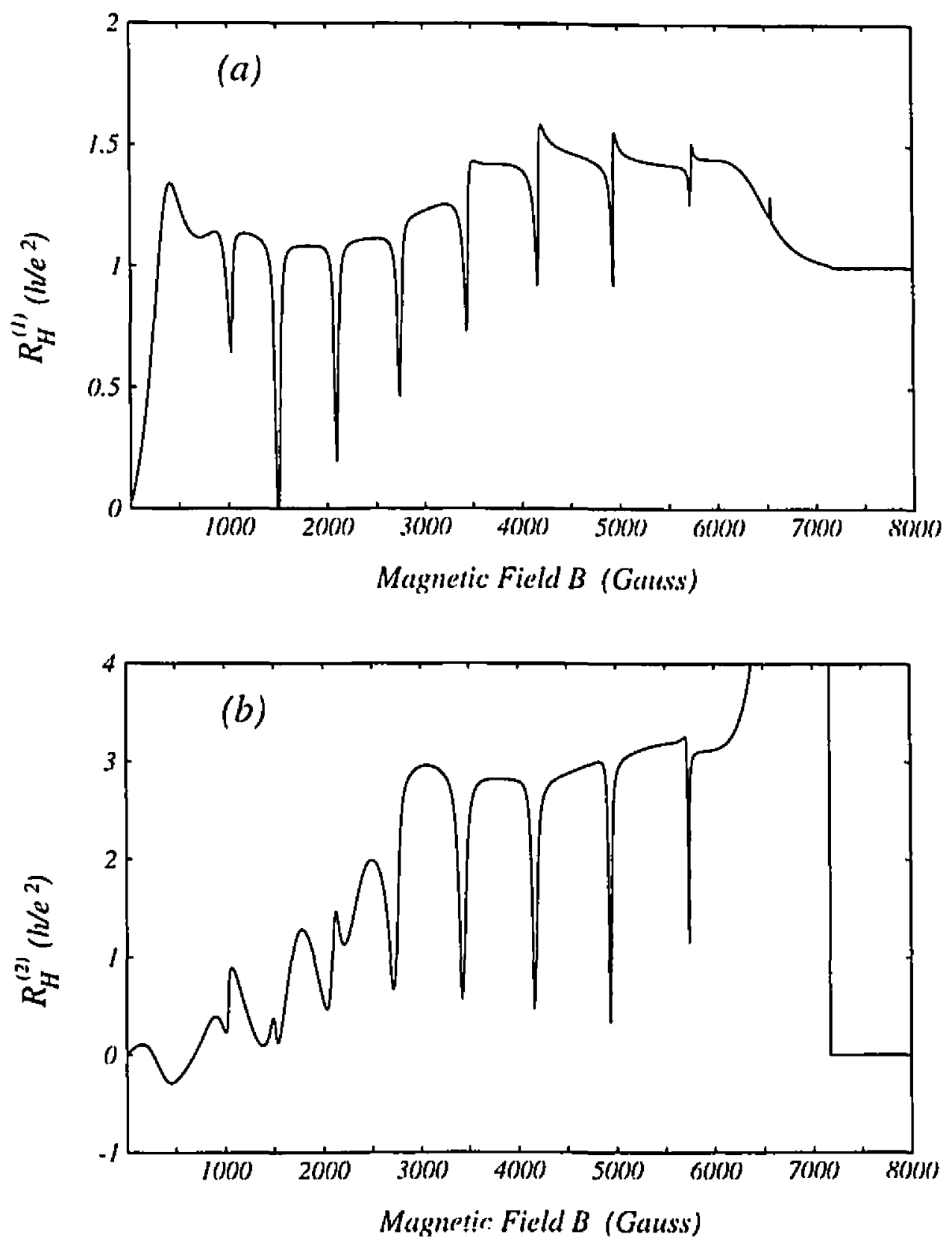

Figure 4.12: Hall resistance $R_{H}$ as a function of magnetic field $B$ for intermediate untidot size $\left(r_{a}=0.5 W\right)$. ( $(a)$ is for channel 1 and (b) is for channel 2). The behavior of $R_{H}$ of (a) is more like Fig. (4.10a) whereas that of (b) is more like Fig. (4.10b). 
The Hall resistance Eq. (4.45) is shown in Fig. (4.10b) as a function of $\chi$. It is also, of course, a periodic function of $\chi$. Let us discuss Fig. (4.10b) in more detail. In the absence of backscattering in the probes, $R_{l}=0$, the conductor in Fig. (4.8b) exhibits no localized state. We have direct transmission along the boundary of the conductor connecting the probes in a cyclical fashion. The Hall resistance is quantized. For small reflection probabilities, corresponding to a localized state that is strongly coupled to the probes, weak oscillation develop around the quantized value (Hall plateau). With increasing reflection probability $R_{l}$, the localized state becomes long-lived. The Hall resistance depends strongly on the phase $\chi$ and shows large excursions above the quantized value. For $\chi=0$, i.c., at resorance, the Hall resistance becomes very small, and for reflection probabilities $R_{l}$ close to $1, R_{H}$ is zero. Thus, as Büttiker has pointed out that it is possible to quench the Hall effect even at very high fields.

At this moment, based on the properties of Hall resistance in Fig. (4.10), we can give a direct demonstration for the topological explanation of the crossover regime. In Fig. (4.11) we show the calculation result of $R_{H}^{1}$ and $R_{H}^{2}$ for different antidot sizes $r=W / 4, W / 2$, where $R_{H}^{1}, R_{H}^{2}$ represent the Hall resistance when the incoming electron is in channel 1 and channel 2 respectively. For a small antidot $\left(r_{a}=0.25 W\right)$, when we compare Fig. (4.10a) and Fig. (4.11a,b), we can conclude that at this size the carrier transport are similar to pattern (a) of Fig. (4.8) no matter which channel the incoming carrier belongs to. If we increase the radius of the antidot, for instance to $r_{a}=0.5 \mathrm{~W}$, we find that the behavior of $R_{H}^{1}$ (Fig. (4.12a)) is more like Fig. (4.10a) while that of $R_{H}^{2}$ (Fig. (4.12b)) prefers Fig. (4.10b); this just illustrates the following relation which can be seen from Fig. (4.9).

$$
\alpha^{(a)}>\beta^{(a)}, \beta^{(b)}>\alpha^{(b)} .
$$

Thus we have given a direct although not stringent demonstration of our assumption (Fig. (4.9)) with the help of resonant behavior of Hall resistance of two important transport patterns.

\subsection{Universality of Micro-symmetry in Crossover Regime}

In this section, we investigate the universality of the "micro-symmetry" in the crossover regime which is an exciting result we got in $\S 4.3$. Our main task in this section is 
to answer the following question: is the micro-symmetry a universal property for a general structure without the dependence on the particular geometrical shape of the sample?

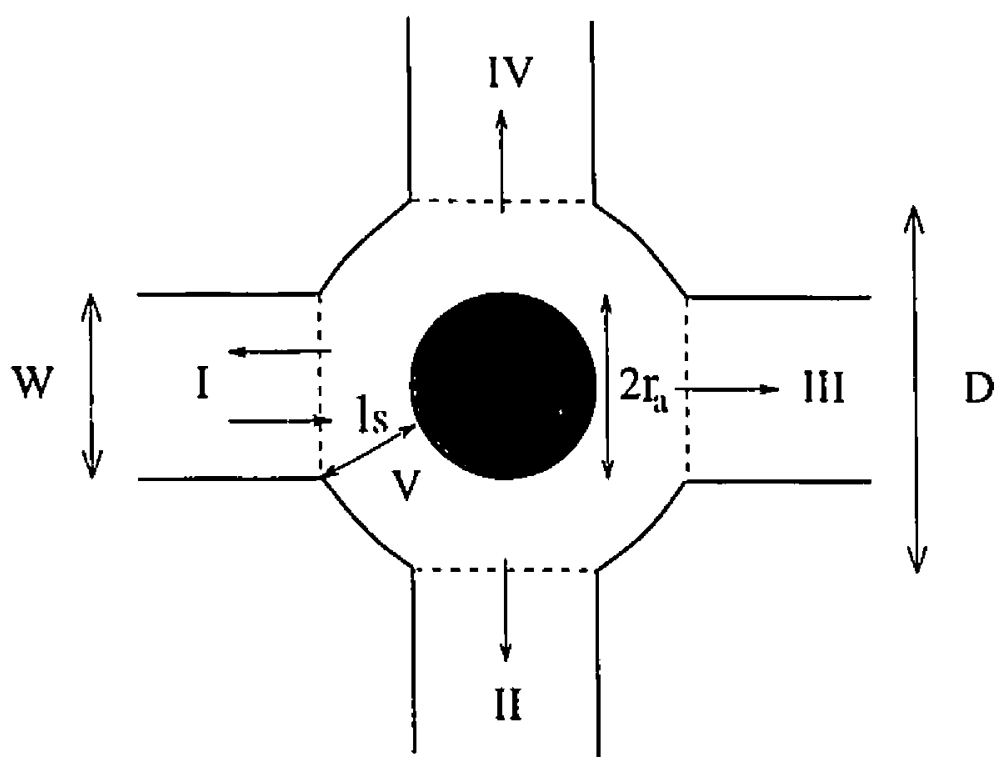

Figure 4.13: Schematic plot of the Hall junction. An antidot of radius $r_{a}$ is confined inside the circular quantum dot. Electrons incident from probe $I$.

First, let us consider the situation where the geometry shape of the quantum dot is changed. Here the numerical calculation method of the previous section is applied to compute the transmission coefficients as a function of the size of the antidot for a circular-shaped structure at a fixed magnetic field, as shown in Fig. (4.13). The Hall junction is now a circular quantum dot instead of a square one, a circular antidot confined inside the quantum dot and four probes connected to the quantum dot forming a Hall junction. The values of $W$ and $D$ are the same as those in section \$ 4.3. The incoming electron energy is still fixed by its momentum $k W=9.5$ which is just above the 3rd subband energy when $B=0$. $B$ is fixed at 6060 Gauss. That means the reservoirs are populated by carriers up to the second quantum channel and in the particular magnetic field we selected, only the first two modes can propagate.

Fig (4.14a) shows the transmission coefficient $T_{m 1},(m=1,2,3,4)$ as a function 

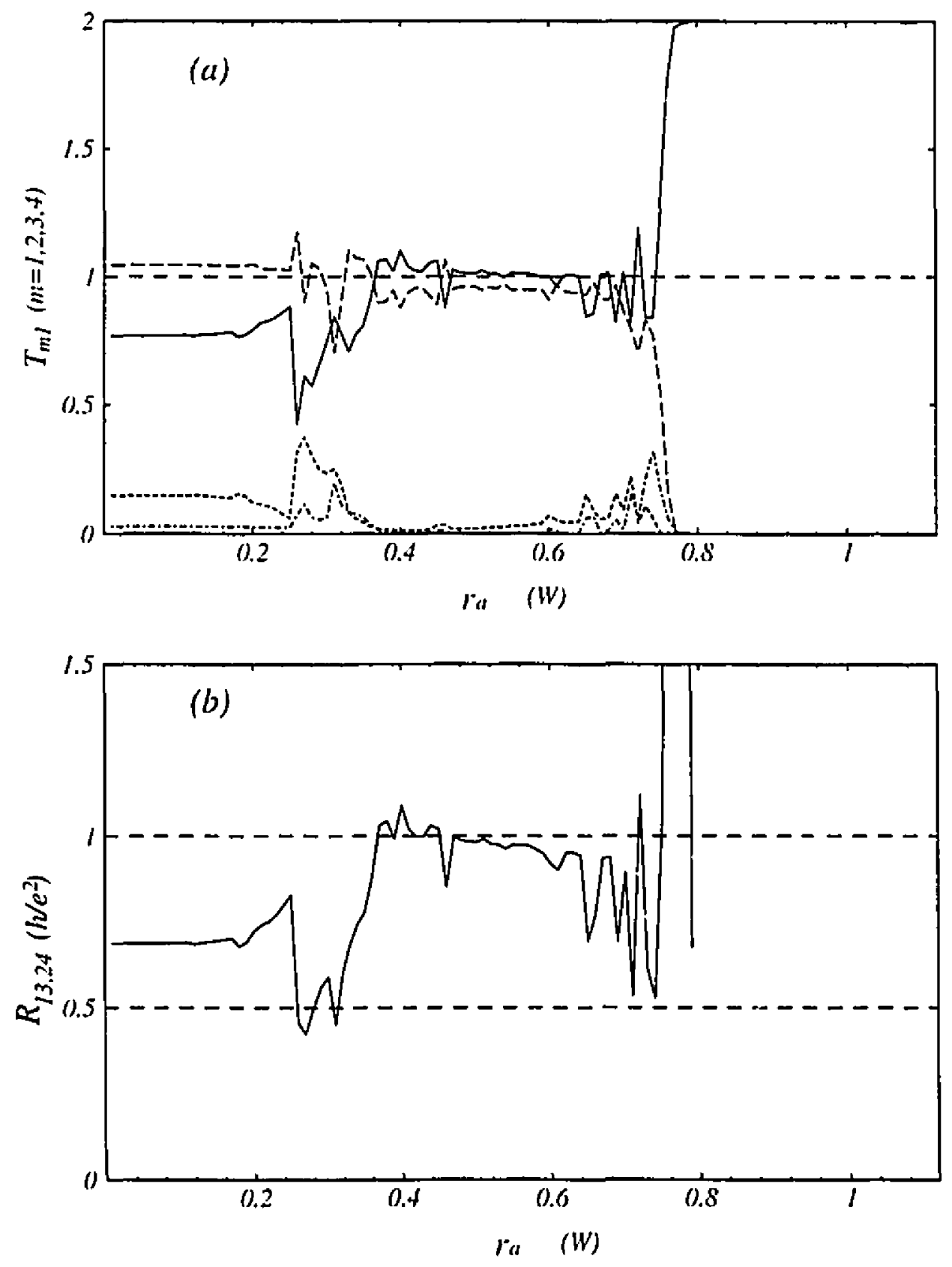

Figure 4.14: (a) Transmission coefficients $T_{m n}$ of circular-shaped Hall junction as a function of the antidot size $r_{a}$ at $B=6060$ Gauss, where subseript $m n$ indicating transmission from probe $n$ to probe $m$. Note in the crossover regime $T_{11} \approx T_{21} \approx 1$ while $T_{31} \approx T_{11} \approx 0$. Solid line is $T_{11}$, dashed line is $T_{21}$, short-dashed line is $T_{31}$ and dot-dashed line is $T_{11}$. (b) Hall resistance $R_{H}$ as a function of the antidot size $r_{\mathrm{a}}$ at $B=6060$ Gauss. It is obvious that there is a crossover regime where Hall resistance $R_{H}$ takes a abnormal integer vulue of $h / e^{2}$,i.e., $N \approx 1$. 
of the radius $r_{a}$ of the antidot where $T_{m 1}$ is the total transmission coefficient from probe I to probe $m$ including all the two propagating modes. From Fig. (4.14a) we can find that there is a regime with intermediate values of $r_{a}\left(0.45 W<r_{a}<0.65 W\right)$ where the following relations approximately hold:

$$
T_{11} \approx 1, T_{21} \approx 1, T_{31} \approx 0, T_{11} \approx 0
$$

These can approximately give the unusual Hall plateau $R_{H}=h / e^{2}$ when transmission edge states reciprocally propagate. Inspecting Fig. (4.15), we shall obtain similar formula as Eq. (4.8)

$$
T_{11}^{1} \approx T_{21}^{2}, T_{21}^{1} \approx T_{11}^{2}, T_{31}^{1} \approx T_{11}^{2}, T_{41}^{1} \approx T_{31}^{2}
$$

where $\stackrel{P}{m n}_{m}^{i}$ has the same meaning as that in $\S 4.4$. Since $T_{31}^{i}$ and $T_{11}^{i}$ are almost zero in the crossover regime, they are not shown in Fig. (4.15). So, in the crossover regime of the structure shown in Fig. (4.13), our topological argument is still approximately valid (Eq. (4.36)).

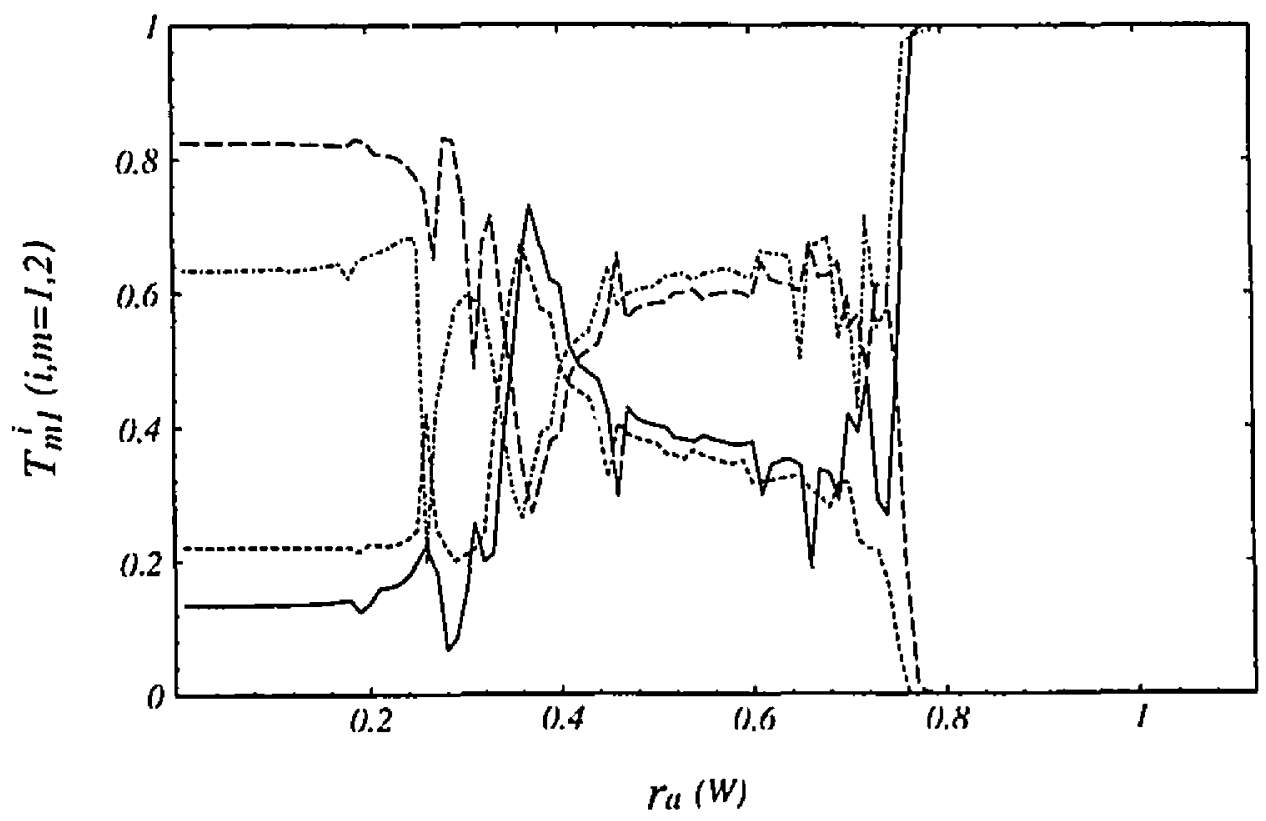

Figure 4.15: Transmission coefficients of circular- shaped Hall junction of individual incoming channels $T_{m n}^{i}$, where $i$ is the channel number, as a function of $r_{a}$ at $B=6060$ Gauss. Note in the crossover regime $T_{11}^{1} \approx T_{2}^{3} . T_{21}^{1} \approx T_{11}^{2}$. Solid line is $T_{11}^{1}$, dashed line is $T_{21}^{1}$, shortdashed line is $T_{21}^{2}$ and dot-dashed line is $T_{11}^{2}$ 
The interesting thing about Fig. $(4.14 \mathrm{a})$ is that for a small antidot $\left(r_{a}<0.45 \mathrm{~W}\right)$, we don't have $T_{21}=2$ and $T_{11}=0$ as we expect. On the contrary, the transmissions take the following values $T_{21} \approx 1.05, T_{11} \approx 0.77, T_{31} \approx 0.15, T_{41} \approx 0.03$.

So, it is rather clear that the transmission properties within crossover regime do not strongly depend on the shape of the Hall junction whereas the properties in the small-antiriot-size regime do.

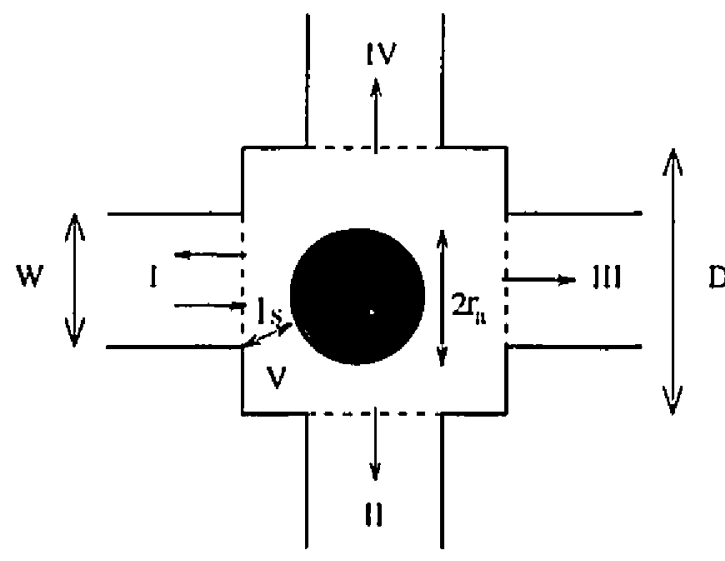

(a)

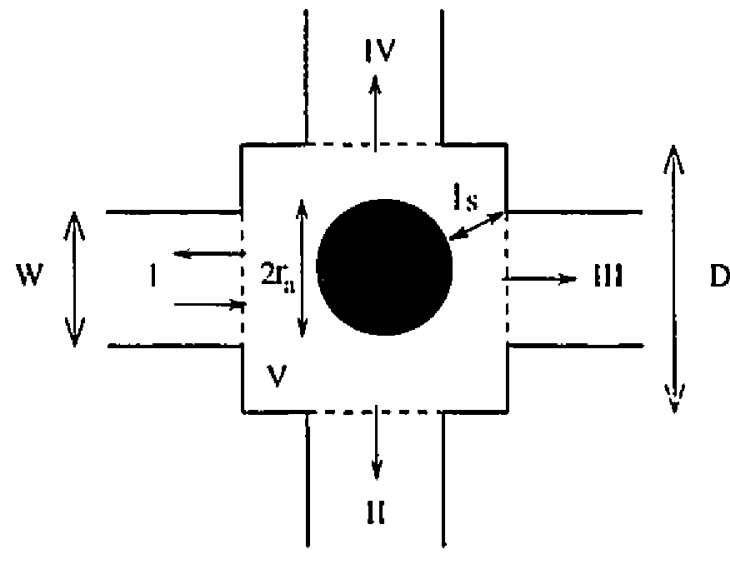

(c)

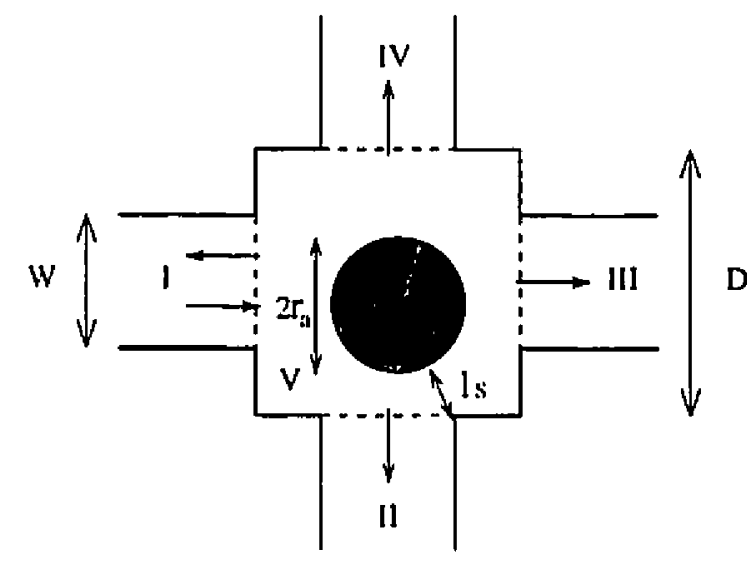

(b)

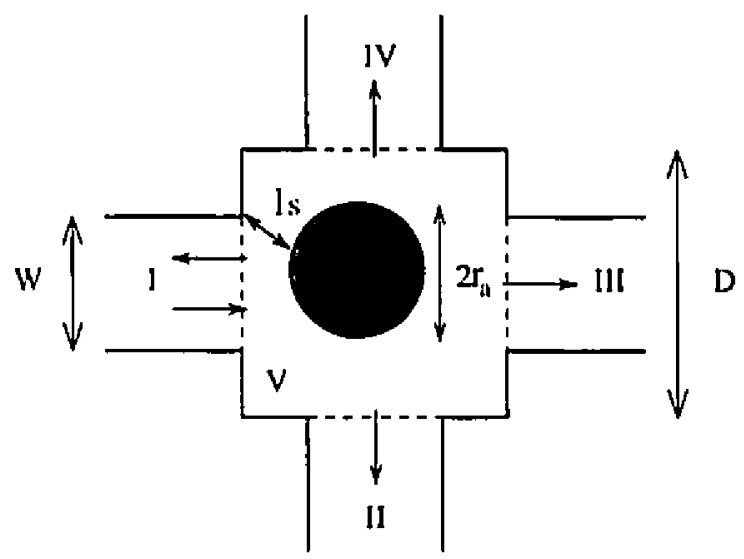

(d)

Figure 4.16: Schematic plot of the Hall junctions. An antidot of radius $r_{a}$ is confined inside the square quantum dot. Electrons incident from probe I. The centers of the antidots of structure (a), (b), (c) and (d) are located at points $(-W / 2,-W / 2),(W / 2,-W / 2),(W / 2, W / 2)$ and $(-W / 2, W / 2)$ respectively. $W$ is the width of the probes and point $(0,0)$ is the center of the Hall junctions. 
The above numerical results shows that the appearance of crossover regime and the correctness of Eqs. (4.7) and (4.8) are quite universal and almost do not depend on the particular structure we choose. Furthermore, our topological explanation of the micro-symmetry in the crossover regime (see $§ 4.5$ ) does not rely on the exact position where the antidot is. Hence it is quite natural to ask a further question: do we still have that symmetry when the antidot is no longer located in the middle of the Hall junction?

Consider the Hall junction illustrated in Fig. (4.16a). All are the same us before (see Fig. (4.2)) expect the center of the antidot is now located at the position $(-W / 10,-W / 10)$ (assuming the center of the whole structure is at $(0,0)$ ). 'The magnetic field is fixed at $B=6000$ Gauss which belongs to the "crossover regime of $\mathrm{B}$ ". Our numerical calculation seems to provide a positive answer to the above question. Fig. (4.17a) shows the transmission coefficient $T_{m 1},(m=1,2,3,1)$ which unambiguously demonstrates that Eq. (4.7) still holds here. To test our topological explanation, the validness of Eq. (4.8) is required which is indeed the case (see Fig. (4.17b)). Since the center of the antidot is a four-fold symmetric point, we need to calculate the transmission coefficients of all the other three cases (Fig. $(4.16 \mathrm{~b}, \mathrm{c}, \mathrm{d})$ ) to get the Hall resistance $R_{H}$ by making use of the Büttiker formula Eq. (4.1). Figs. (4.18), (4.19) and (4.20) are the counterparts of Fig. (4.17) and show that Eqs. (4.7) and (4.8) are also valid for the other three cases.

Now let us calculate the Hall resistance $R_{H}$ for the structure of Fig. (1.16a) when an electron is incoming from probe I. Considering the topological symmetry of the four structures in Fig. (4.16), the transmission probabilities of conductors in Fig. (4.16b,c,d) can be obtained by a permutation of the transmission probabilities of the conductor in Fig. (4.16a). In particular, for the transmission coefficients we find

$$
T_{m n}^{(\alpha)}=T_{m+5-n, 1}^{(\alpha+5-n)},(m, n=1,2,3,4 ; \alpha=a, b, c, d),
$$

where $T_{m n}^{(\alpha)}$ is the transmission coefficient from probe $n$ to probe $m$ of structure $(\alpha)$ of Fig. (4.16) and where the index of $T_{m n}^{(\alpha)}$ is taken to be modulo 4. For the sake of discussion here we employ another form of Büttiker formula (equivalent to Eq. (4.1)) [27],

$$
R_{m n, k l}=\frac{\alpha_{21}}{\alpha_{11} \alpha_{22}-\alpha_{12} \alpha_{21}},
$$



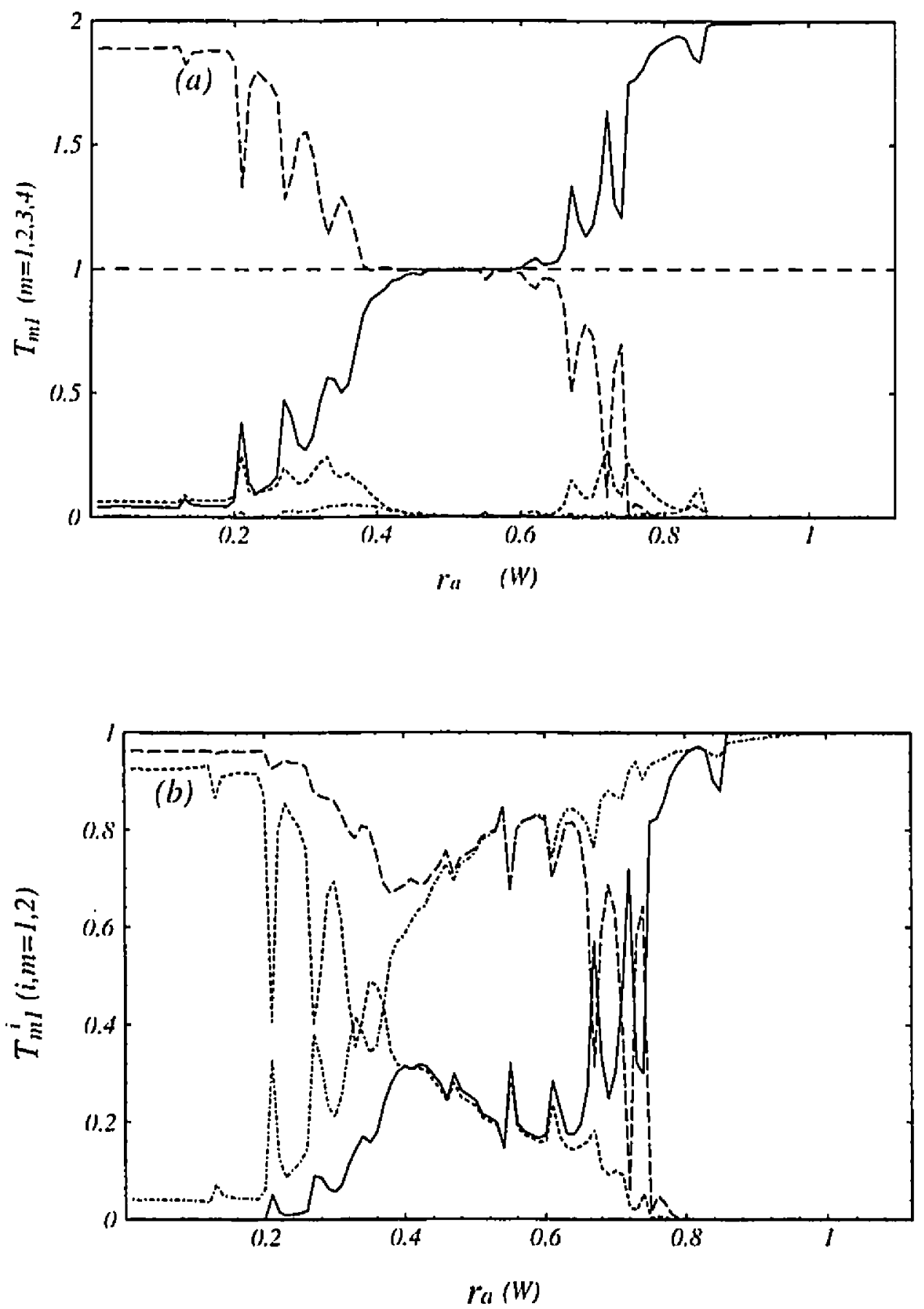

Figure 4.17: Transmission coefficients of the structure shown in Fig. (4.16a). (a) Transmission coefficients $T_{m n}$ as a function of the antidot size $r_{\mathrm{a}}$ at $B=6060$ Gauss, where subscript $m n$ indicating transmission from probe $n$ to probe $m$. Note in the crossover regime $T_{11}=T_{21}=1$ while $T_{31}=T_{41}=0$. Solid line is $T_{11}$, dashed line is $T_{21}$, short-dashed line is $T_{31}$ and dot-dashed line is $T_{41}$. (b) Transmission coefficients of individual incoming channels $T_{m n}^{i}$, where $i$ is the channel number, as a function of $r_{a}$ at $B=6060$ Gauss. Note in the crossover regime $T_{11}^{1}=T_{21}^{2}, T_{21}^{1}=T_{11}^{2}$. Solid line is $T_{11}^{1}$, dashed line is $T_{21}^{1}$, shortdashed line is $T_{21}^{2}$ and dot-dashed line is $T_{11}^{2}$. 

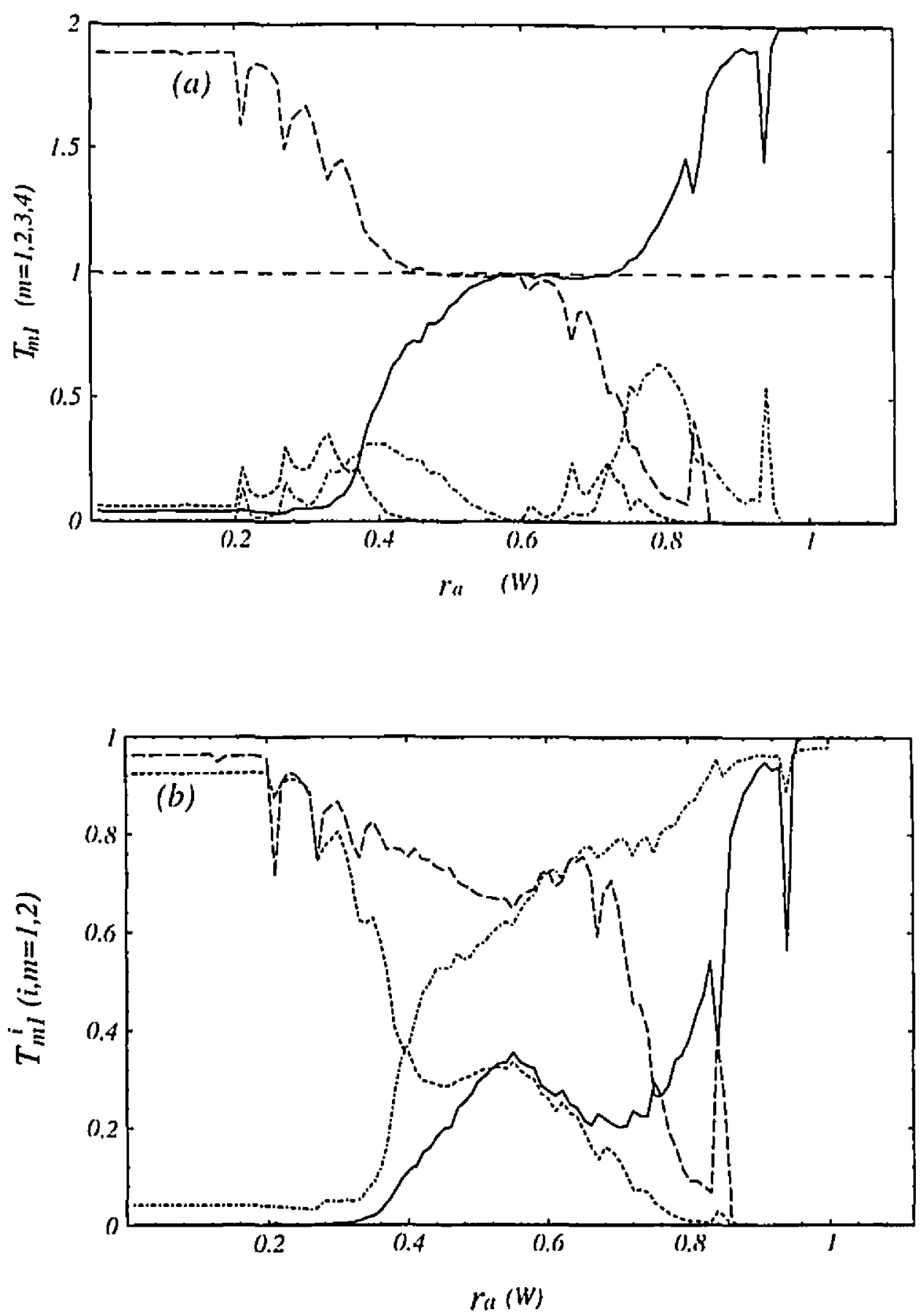

Figure 4.18: Transmission coefficients of the structure shown in Fig. (4.16b). (a) Transmission coefficients $T_{m n}$ as a function of the antidot size $r_{a}$ at $B=6060$ Gauss, where subscript $m n$ indicating transmission from probe $n$ to probe $m$. Note in the crossover regime $T_{11}=T_{21}=1$ while $T_{31}=T_{41}=0$. Solid line is $T_{11}$, dashed line is $T_{21}$, short-dashed line is $T_{31}$ and dot-dushed line is $T_{41}$. (b) Transmission coefficients of individual incoming channels $T_{m n}^{i}$, where $i$ is the channel number, as a function of $r_{a}$ at $B=6060$ Gauss. Note in the crossover regime $T_{11}^{1}=T_{21}^{2}, T_{21}^{1}=r_{11}^{2}$. Solid line is $T_{11}^{1}$, dashed line is $T_{21}^{1}$, shortdashed line is $T_{21}^{2}$ and dot-dashed line is $T_{11}^{2}$. 

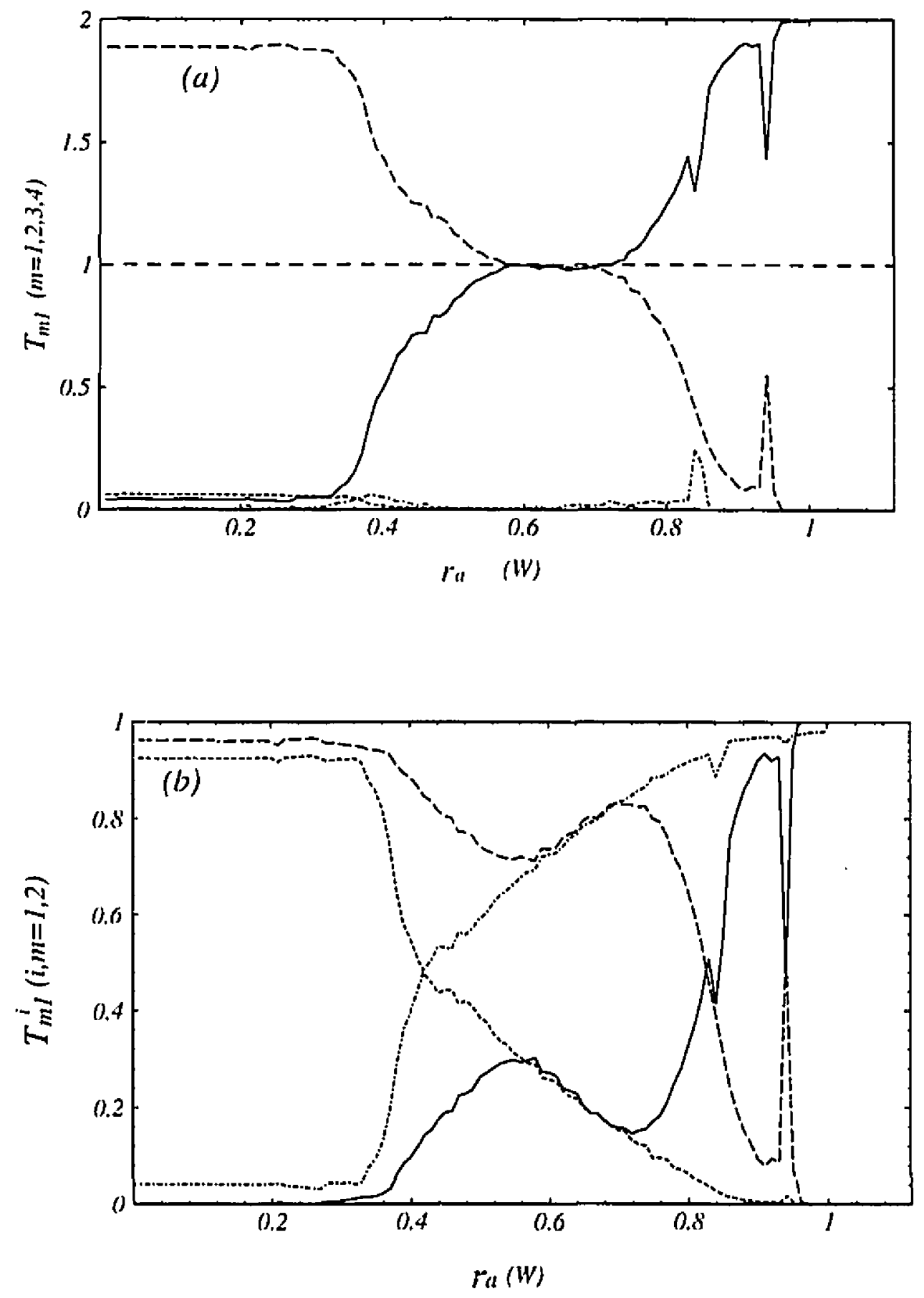

Figure 4.19: Transmission coefficients of the structure shown in Fig. (4.16c). (a) Transmission coefficients $T_{m n}$ as a function of the antidot size $r_{a}$ at $B=6060$ Gauss, where subscript $m n$ indicating transmission from probe $n$ to probe $m$. Note in the crossover regime $T_{11}=T_{31}=1$ while $T_{31}=T_{41}-0$. Solid line is $T_{11}$, dashed line is $T_{21}$, short-dashed line is $T_{31}$ and dot-dashed line is $T_{41}$. (b) 'lransmission coefficients of individual incoming channels $T_{m n}^{i}$, where $i$ is the channel number, as a function of $r_{a}$ at $B=6060$ Gauss. Note in the crossover regime $T_{11}^{1}=T_{21}^{2}, T_{31}^{12}=T_{11}^{2}$. Solid line is $T_{12}^{2}$, dashed line is $T_{21}^{2}$, shortdashed line is $T_{21}^{2}$ and dot-dashed line is $T_{11}^{2}$. 

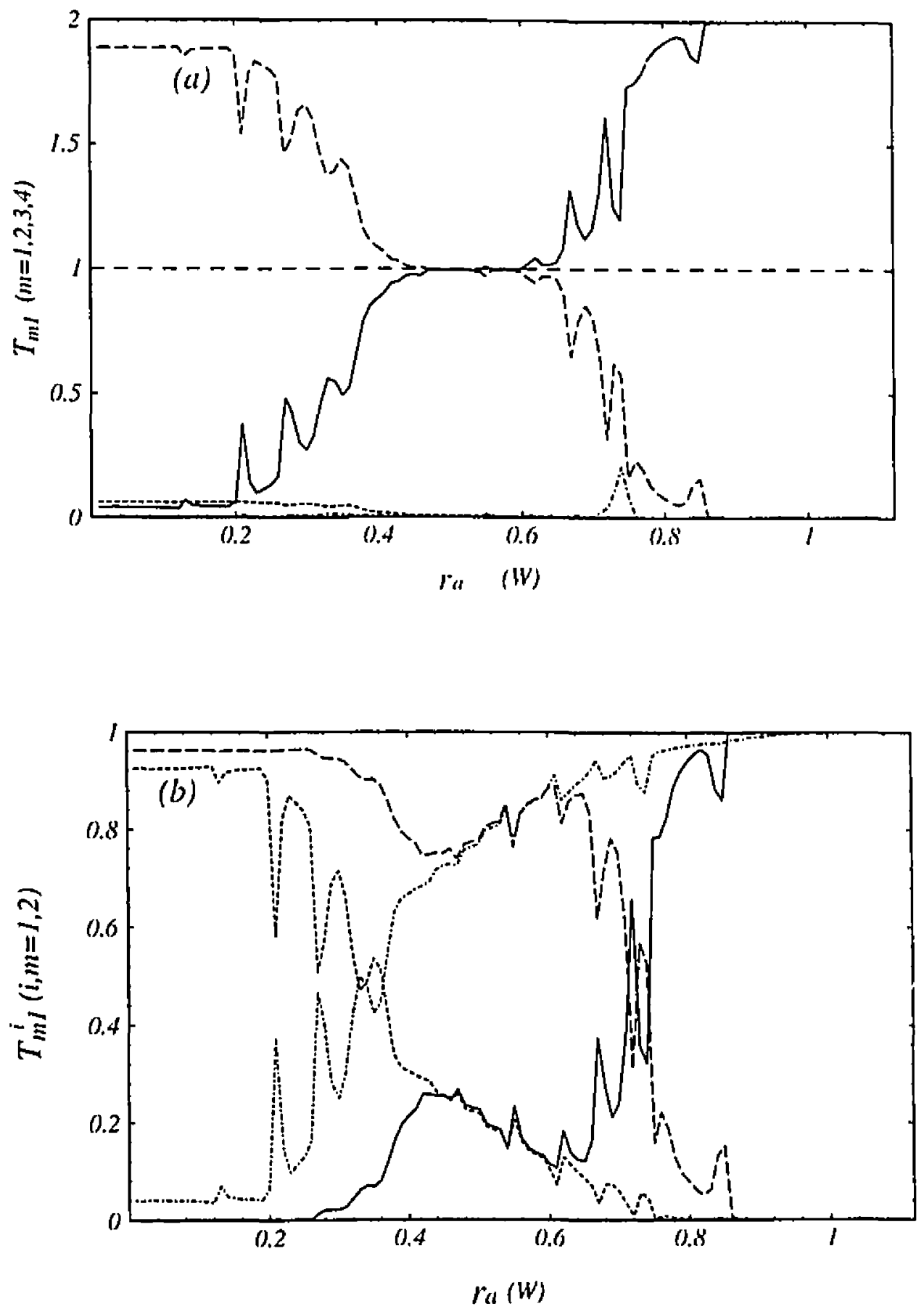

Figure 4.20: Transmission coefficients of the structure shown in Fig. (4.10d). (a) Transmission coefficients $T_{m n}$ as a function of the antidot size $r_{a}$ at $B=6060$ Gauss, where subscript $m n$ indicating transmission from probe $n$ to probe $m$. Note in the crossover regime $T_{11}=T_{21}=1$ whilc $T_{31}=T_{41}=0$. Solid line is $T_{11}$, dashed line is $T_{21}$, short-dashed line is $T_{31}$ and dot-dashed line is $T_{41}$. (b) Transmission coefficients of individual incoming channcis $T_{m n}^{i}$, where $i$ is the channel number, as a function of $r_{a}$ at $B=6060$ Gauss. Note in the crossover regime $T_{11}^{1}=T_{11}^{2}, T_{21}^{2}=T_{11}^{2}$. Solid line is $T_{11}^{1}$, dashed line is $T_{21}^{1}$, shortdashed line is $T_{21}^{2}$ and dot-dashed line is $T_{11}^{2}$. 
where

$$
\begin{gathered}
\alpha_{11}=\frac{e^{2}}{h}\left[\left(N-T_{m m}\right) S-\left(T_{m l}+T_{m k}\right)\left(T_{l m}+T_{k m}\right)\right] / S, \\
\alpha_{12}=\frac{e^{2}}{h}\left[T_{m k} T_{n l}-T_{m l} T_{n k}\right] / S, \\
\alpha_{21}=\frac{e^{2}}{h}\left[T_{k m} T_{l n}-T_{l m} T_{k n}\right] / S, \\
\alpha_{22}=\frac{e^{2}}{h}\left[\left(N-T_{k k}\right) S-\left(T_{k m}+T_{k n}\right)\left(T_{n k}+T_{m k}\right)\right] / S,
\end{gathered}
$$

and

$$
S=T_{m k}+T_{m l}+T_{n k}+T_{n l}=T_{k m}+T_{l m}+T_{k n}+T_{l n}
$$

Here $N$ is the number of quantum channel occupied by the incident electron.

Fig. (4.21) shows the dependence of Hall resistance $R_{H}$ on the radius of antidot $r_{a}$ corresponding to the four Hall-bar structures shown in Fig. (4.16) respectively with incoming electron approaching the Hall junction from probe $\mathrm{I}$. It is obvious that the "mysterious" crossover regime still exists in every situation which give us confidence to say that the "micro-symmetry" we observed is universal vithin the scope of our calculations. If we inspect Fig. (4.21) carefully, we shall find the behavior of Hall resistance of structure (a) and (c) are quite similar and so are the ones of structures (b) and (d). Since structures (a) and (c), (b) and (d) are center symmetric respectively, we obtain an interesting property,

$$
R_{H}(\boldsymbol{r}) \approx R_{H}(-\boldsymbol{r})
$$

where $r$ is the position of tine center of the antidot. Of course, here we assume that the antidot to be a center symmetric one. This feature is the natural result of the permutation relation Eq. (4.49).

In summary the Hall resistance was studied for both a circular-shaped and square quantum dots with a non-centered antidot. It is shown that, in both cases, we can still find a crossover regime where the two incoming edge states are both reciprocally transmitting which can lead to the abnormal Hall plateau before the perfect quantum Hall regime is reached. However we emphasis that what we have done is a numerical experiment for the appearance of the crossover regime over several particular structures and this is not a fundamental proof of this phenomenon. It will be 
interesting and challenging to analytically derive the formula for transmission coefficients in order to find the "micro-symmetry" which is the base of the appearance of the crossover regime.
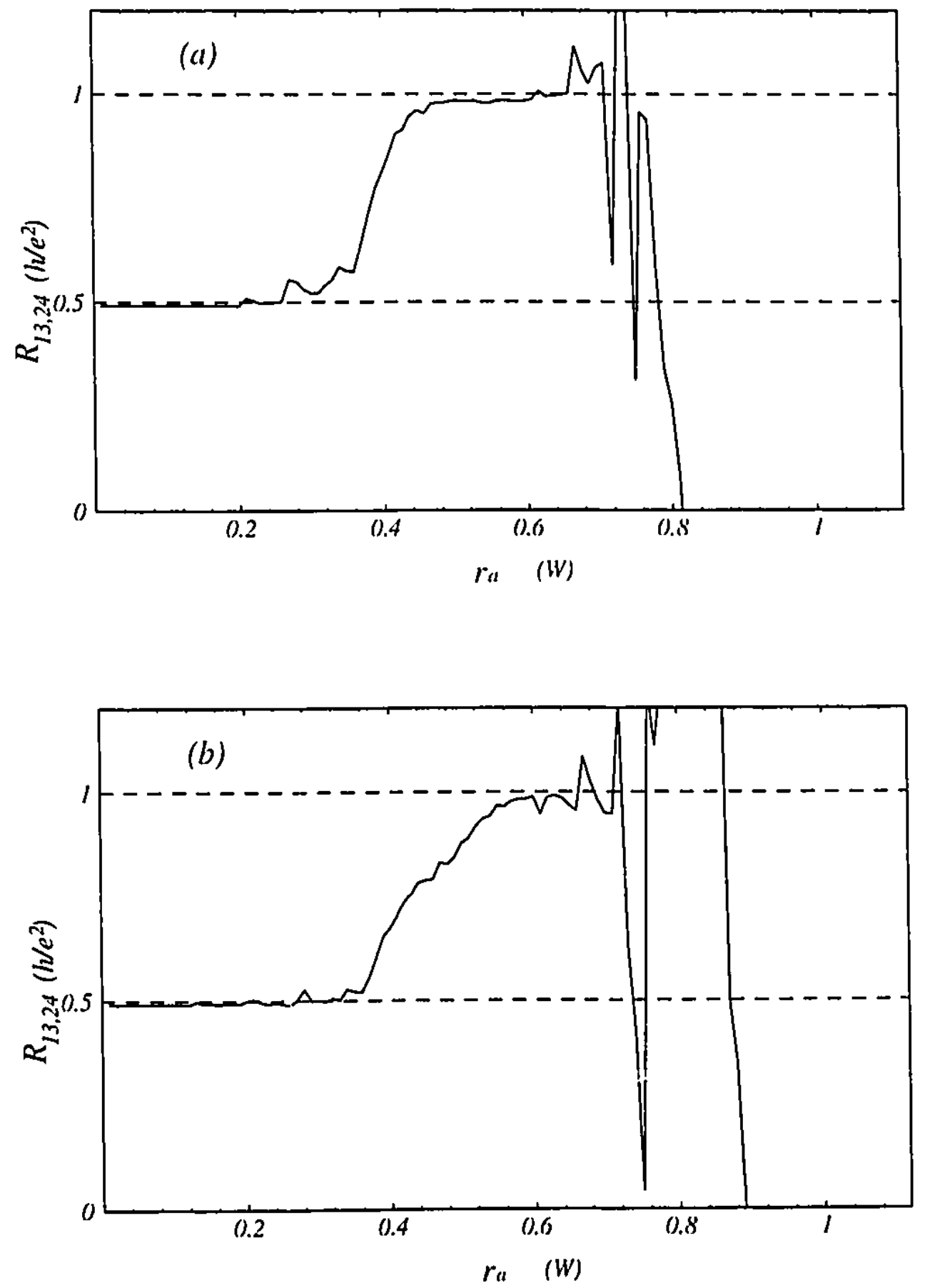

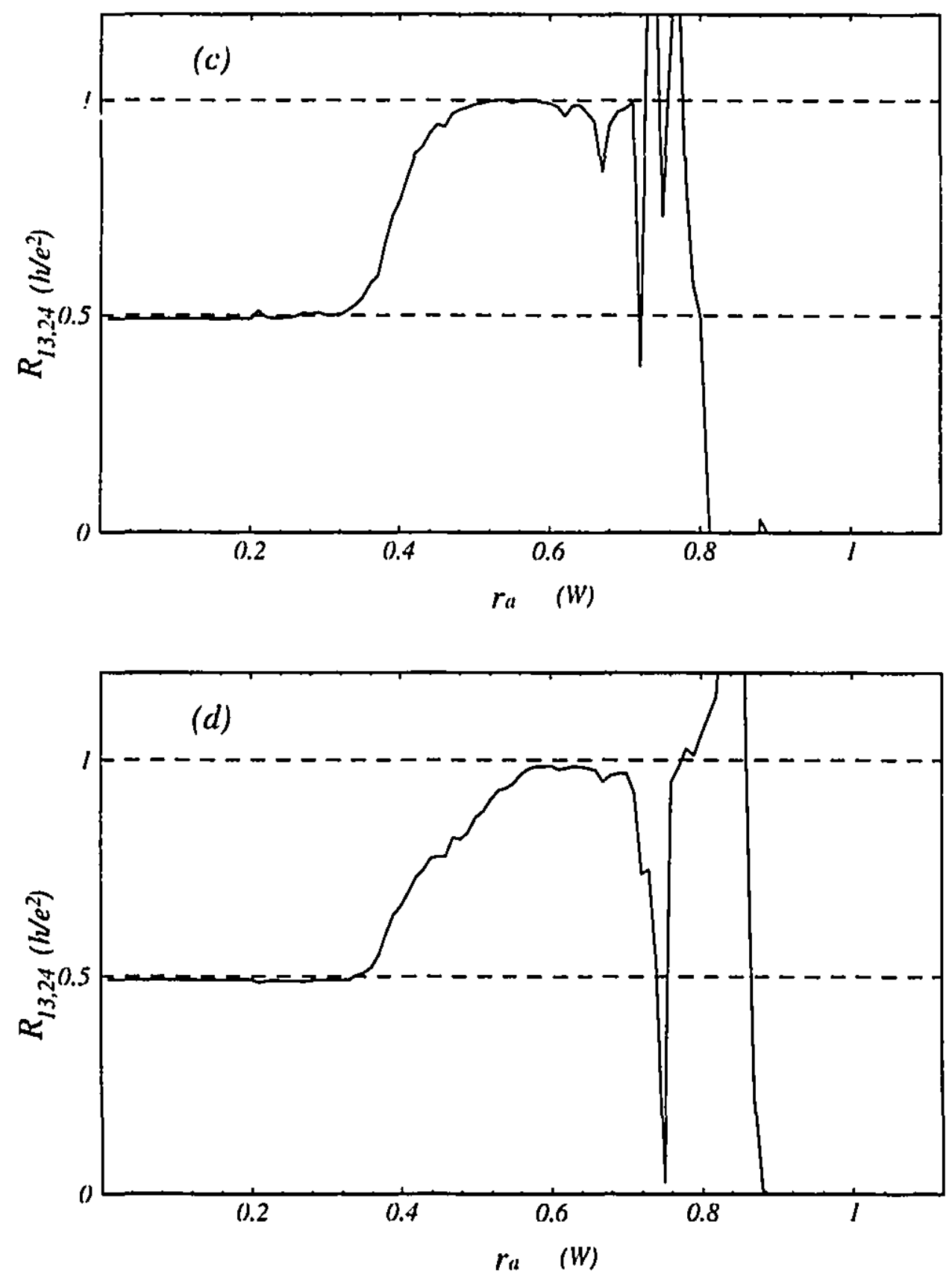

Figure 4.21: Hall resistance $R_{H}$ as a function of the antidot size $r_{a}$ at $B=6060$ Gauss. It is obvious that there is a cro sover regime where Hall resistance $R_{H}$ takes a abnormal integer value of $h / e^{2}, i . e$, $N=1$. (a), (b), (c) and (d) are for Hall junctions shown in Fig. (4.16a, b, c and d) respectively. 


\section{5}

\section{CONCLUSIONS}

In this thesis, we have studied in detail the size effect of an artificial impurity or an antidot confined in a quantum Hall junction in the ballistic transport regime [30]. It was an unsolved problem regarding how a two dimensional electron gas transforms from the quantum Hall regime (strong magnetic field) to the weak magnetic field regime. Here we investigated this problem from another equivalent point of view by varying the size of the antidot, i.e., the dimension of Hall junction structure instead of varying the magnetic field. The reason this is equivalent is because whether a magnetic field is strong or weak depends on the relative ratio of the constriction length of the structure (see $\S 2.6$ ) and the magnetic length $l_{B}$ (see $\S 2.2$ ). In our problem, no impurity means strong magnetic field while very big impurity represents weak field. Of course, suitable values of energy of the incoming electron and magnetic field strength are required.

Our numerical calculation demonstrated that as the impurity size is increased, transport characteristics change from the usual integer quantum Hall regime to a regime dominated by Aharonov-Bohm oscillations. We have directly demonstrated the formation and coupling of the magnetic edge states, and this lead to the sharp dips in the Hall plateau. An interesting crossover regime is discovered as the antidot size takes intermediate values. In this regime our numerical data shows a lost of unusual symmetries between various transmission coefficients, and these symmetries lead to the appearance of a Hall plateau before the perfect quantum Hall regime is reached. In light of the scattering matrix theory [31] and the topological equivalence argument [24], we proposed a topological explanation for the above "strange" behavior of transmission coefficients in the crossover regime. Our topological analysis, based on the two intrinsic topological equivalence of the dominating transmission 
patterns, indeed produces the observed transmission symmetries. Of course, this topological argument is not unique, but it fits quite well with the data of the numerical calculation and is very simple to use. Through the study of resonant behavior for the two transmission patterns and comparing that with our calculation results of the Hall resistance of different structures have also produced the same "micro-symmetry" behavior.

Since our analytical derivation of the relationships between the transmission coefficients is based on quite general assumptions, we expect the "micro-symmetry" observed in the particular structure (Fig. (4.2)) to be quite general as well. The universality of "micro-symmetry" of the crossover regime is studied from two directions. First, it is established numerically that for a circular Hall junction there is also a crossover regime of antidot size where the symmetry behavior of transmission coefficients approximately hold. But it seems that the general property of transmission coefficients as functions of the size of antidot depend on the geometrical shape of the Hall junction. Second, the transmission probabilities as a function of antidot size are investigated for a square Hall junction with an antidot not locating at the center (the displacement of the center of the antidot is of the order of one tenth of the width of the probes). The symmetry properties of crossover regime hold very well although the shape of the Hall resistance $R_{H}$ is not exactly the same as that of the centered-antidot structure.

Magneto-conductance fluctuations in mesoscopic conductors have many interesting behavior, and with the ability of controlling the location and size of an artificial impurity, further interesting physics will certainly be seen and understood. While the "micro-symmetry" between the transmission coefficients observed in the numerical calculation can not directly be measured since a measurement involves all transmitting channels, the consequences of this symmetry, such as the behavior of the Hall resistance in the crossover regime, can certainly be tested experimentally. Finally it will be interesting to examine the situation where more than two incoming channels participate transport, and thus a more complicated symmetry pattern may be observed. 


\section{APPENDIX}

\section{A.1 Definitions of Different Transmission Probabilities}

In this thesis we have defined three different transmission probabilities $-T_{i j, m n}$, $T_{m n}^{j}$, and $T_{m n}$. To provide convenience for a reader it will be worth listing all the definitions of these transmission probabilities and showing the relations between them in one place.

\section{Definitions}

$T_{i j, m n}$ : transmission probability of a carrier incident in probe $n$ and channel $j$ to exit in probe $m$ and channel $i$.

$T_{m n}^{j}$ : transmission probability from probe $n$ to probe $m$ when the incoming carrier is in channel $j$.

$T_{m n}$ : total transmission probability from probe $n$ to probe $m$.

\section{Relations}

$$
\begin{gathered}
T_{m n}^{j}=\sum_{i=1}^{M_{m}} T_{i j, m n} \\
T_{m n}=\sum_{j=1}^{M_{n}} T_{m n}^{j}=\sum_{i=1}^{M_{m}} \sum_{j=1}^{M_{n}} T_{i j, m n}
\end{gathered}
$$




\section{BIBLIOGRAPHY}

[1] M. 1. Reed and W.P. Kirk. Nanostructure Physics and Fabricaiion. Academic, New York, 1989.

[2] B. J. van Wees, H. Van Houten, C. W. J. Beenakker, J. G. Williamson, L. P. Kouwenhoven, D. van der Marel, and C. T. Foxon. Phys. Rev. Lett., 60:848, 1988.

[3] D. A. Wharam, T. J. Thornton, R. Newbury, M. Pepper, H. Ahmed, J. E. F. Frost, D. G. Hasko, D. A. Ritchie, and G. A. C. Jones. J. Appl. Phys., 61:4723, 1987.

[4] C.W.J. Bennakker and H. van Houten. In H. Ehrenreich and D. Turnbull, editors, Solid State Physics: Advances in Research and Applications, volume 44, pages 1-228. Academic Press, New York, 1991.

[5] M. Kastner. Phys. Today, 46:24, 1993.

[6] Y. Aharonov and D. Bohm. Phys. Rev., 115:485, 1959.

[7] K. von Klitzing, G. Dorda, and M. Pepper. Phys. Rev. Lett., 45:495, 1980.

[8] K. von Klitzing. Rev. of Mod. Phys, 58:519, 1986.

[9] H. van Houten and C. W. J. Beenakker. In W. Van Haeringen and D. Lenstra, editors, Analogies in Opticas and Microelectronics. Kluwer Academic Press, Dordrecht, 1990.

[10] Y. Imry. In G. Grinstein and G. Mazenko, editors, Directions in Condensed Matter Physics. World Scientific Press, Singapore, 1986.

[11] R.Landauer. IBM Res. Dev, 1:223, 1957.

[12] M. Büttiker. Phys. Rev. Lett, 57:1761, 1986.

[13] T. Ando, A. B. Fowler, and F. Stern. Rev. of Mod. Phys, 54:437, 1982.

[14] D. Heitmann and J.P. Kotthaus. Phys. Today, 46:56, 1993.

[15] B. J. van Wees, L. P. Kouwenhoven, C. J. P. M. Harmans, J. G. Williamson, C. E. Timmering, M. E. I. Broekaart, C. T. Foxon, and J. J. Harris. Phys. Rev. Lett., 62:2523, 1989.

[16] D. Weiss, M. L. Roukes, A. Menschig, P. Grambow, K. von Klitzing, and G. Weimain. Phys. Rev. Lett., 66:2790, 1991. 
[17] R. Fleischmann, T. Geisel, and R. Ketzmerick. Phys. Rev. Lett., 68:1367, 1992.

[18] J. A. Simmons et al. . Phys. Rev. Lett., 63:1731, 1989.

[19] B. I. Halperin. Phys. Rev. B, 25:2185, 1982.

[20] P. Streda, J. Kucera, and A. H. MacDonald. Phys. Rev. Lett., 59:1973, 1987.

[21] J. K. Jain and S. A. Kivelson. Phys. Rev. Lett., 60:1542, 1988.

[22] M. Büttiker. Phys. Rev. B, 38:9375, 1988.

[23] Y. Takagaki and D.K. Ferry. Phys. Rev. B, 48:8152, 1993.

[24] M. Büttiker. Phys. Rev. B, 38:12724, 1989.

[25] G. Kirczenow. Phys. Rev. B, 42:5357, 1990.

[26] J. E. Avron, A. Raveh, and B. Zur. Rev. Mod. Phys., 60:873, 1988.

[27] M. Büttiker. Phys. Rev. Lett., 57:1761, 1986.

[28] C. S. Lent. Appl. Phys. Lett., 67:6353, 1990.

[29] Y. J. Wang, J. Wang, and H. Guo. Phys. Rev. B, 49:1928, 1994.

[30] M. Lei, N. J. Zhu, and H. Guo. Phys. Rev. B, 52:16784, 1995.

[31] W. Porod, Z. Shao, and C. Lent. Appl. Phys. Lett., 61:1350, 1992.

[32] H. Aoki. Rep. Prog. Phys., 50:655, 1987.

[33] R. B. Laughlin. Phys. Rev. B, 23:5632, 1981.

[34] Y. Imry. J. Phys. C, 16:3501, 1983.

[35] Q. Niu and D. J. Thouless. J. Phys. A, 17:2453, 1983.

[36] Q. Niu and D. J. Thouless. Phys. Rev. B, 35:2188, 1988.

[37] D. C. Tsui. In T. Anto and H. Fukuyama, editors, Anderson Localization. Springer-Verlag, Berlin, 1988.

[38] K. von Klitzing. Surf. Sci., 113:1, 1982.

[39] R. Landauer. Phil. Mag., 21:863, 1970.

[40] G. Timp, P. M. Mankiewich, P. de Vegvar, R. Behringer, J. E. Cunningham, R. E. Howard, H. U. Baranger, and J. K. Jain. Phys. Rev. B, 33:6227, 1989.

[41] G. Timp, A. M. Chang, J. E. Cunningham, T. Y. Chang, P. M. Mankiewich, R. Behringer, and R. E. Howard. Phys. Rev. Lett., 58:2814, 1987. 
[42] S. Datta, M. R. Mellock, S. Bandyopadhyay, R. Noren, M. Vazirir, M. Miller, and R. Reifenberger. Phys. Rev. Lett., 55:2344, 1985.

[43] J. K. Jain. Phys. Rev. Lett., 60:2074, 1988.

[44] M. Büttiker. In W. P. Kirk and M. Reed, editors, proc. Int. Symp. on Nanostructure Physics and Fabrication, page 319. Academic Press, New York, 1989.

[45] C. J. G. Ford, T. J. Thornton, R. Newbury, M. Pepper, H. Ahmed, C. T. Foxon, J. J. Harriz, and C. Roberts. J. Phys. C, 21:L325, 1988.

[46] C. J. B. Ford, T. J. Thornton, R. Newbury, M. Pepper, H. Ahmed, D. C. Peacock, D. A. Ritchie, J. E. F. Frost, and G. A. C. Jones. Appl. Phys. Lett., 54:21, 1989.

[47] B. J. van Wees, E. M. M. Willems, C. J. P. M. Harmans, C. W. J. Beenaker, H. van Houten, J. G. williamson, C. T. Foxon, and J. J. Harris. Phys. Rev. Lett., $62: 1181,1989$.

[48] S. komiyama, H. Hirai, S. Sasa, and S. hiyamizu. Phys. Rev. B, 40:12566, 1989.

[49] M. Büttiker, Y. Imry, R. Landauer, and S. Pinhas. Phys. Rev. B, 31:6207, 1985.

[50] M. Ya. Azbel. J. Phys. C, 14:L225, 1981.

[51] M. Büttiker, Y. Imry, and R. Landauer. Phys. Lett. A, 96:365, 1983.

[52] Y. Gefen, Y. Imry, and M. Ya. Azbel. Phys. Rev. Lett., 52:129, 1984.

[53] M. Büttiker, Y. Imry, and M. Ya. Azbel. Phys. Rev. A, 30:1982, 1984.

[54] M. Büttiker. Phys. Rev. B, 32:1846, 1985.

[55] H. L. Engquist and P. W. Anderson. Phys. Rev. B, 24:1151, 1981.

[56] M. Büttiker. Phys. Rev. B, 33:3020, 1986.

[57] M. Büttiker. IBM J. Res. Develop., 32:63, 1988.

[58] H. F. Cheung, Y. Gefen, and E. K. Riedel. IBM J. Res. Develop., 32:359, 1988.

[59] D. Sokolovski. Phys. Lett. A, 123:381, 1988.

[60] C. W. J. Beenakker and H. van Houten. Phys. Rev. B, 39:10445, 1989.

[61] S. Datta. Phys. Rev. B, 40:5830, 1989.

[62] P. H. Beton, B. R. Snell, P. C. Main, J. R. Owers-Bradley, L. Eaves, M. Henini, O. H. Hughes, S. P. Beaumont, and C. D. W. Wilkinson. J. Phys. C, 1:7505, 1989.

[63] A. D. Benoit, S. Washburn, C. P. Umbach, R. P. Laibowitz, and R. A. Webb. Phys. Rev. Lett., 57:1765, 1986. 
[64] H. B. G. Casimir. Rev. Mod. Phys., 17:343, 1945.

[65] D. S. Fisher and P. A. Lee. Phys. Rev. E, 23:6851, 1981.

[66] P. W. Anderson, D. J. Thouless, E. Abrahams, and D. S. Fisher. Phys. Rev. $E$, 22:3519, 1980.

[67] Y. Feng et al. Appl. Phys. Lett., 63:3191, 1993.

[68] G. Kirczenow, A.S. Sachrajda, Y. Feng, R.P. Taylor, L. Henning, J. Wang, P. Zawadzki, and P.T. Coleridge. Phys. Rev. Lett., 72:2069, 1994.

[69] M. Büttiker. Phys. Rev. Lett., 62:229, 1989.

[70] unpublished. C. Gould et al.

[71] M. C. Gutzwiller. Chaos in Classical and Quantum Mechanics. Springer-Verlag, New York, 1990.

[72] Yongjiang Wang, Ning Jia Zhu, Jian Wang, and unpublished. Hong Guo.

[73] Yongjiang Wang, Jian Wang, Hong Guo, and Chris Roland. J. Phys.: Condens. Matter, 6:L143, 1994.

[74] H. U. Baranger, D. P. DiVincenzo, R. A. Jalabert, and A. D. Stone. Phys. Rev. $B, 44: 10637,1991$.

[75] R. L. Schult, H. W. Wyld, and D. G. Ravenhall. Phys. Rev. B, 38:12760, 1990.

[76] M. L. Roukes, A. Scherer, S. J. Allen, H. G. Craighead Jr., R. M. Ruthen, E. D. Beebe, and J. P. Harbison. Phys. Rev. Lett., 59:3011, 1987.

[77] C. J. B. Ford, S. Washburn, M. Büttiker, C. M. Knoedler, and J. M. Hong. Surface Science, 229:298, 1990.

[78] C. J. B. Ford, T. J. Thornton, R. Newbury, M. Pepper, H. Ahmed, D. C. Peacock, D. A. Ritchie, J. E. F. Frost, and G. A. C. Jones. Appl. Phys. Lett., 54:21, 1987.

[79] R. J. Brown, C. G. Smith, M. Pepper, R. Newbury, H. Ahmed, D. G. Hasko, J. E. F. Frost, D. C. Peacock, D. A. Ritchie, and G. A. C. Jones. J. Phys. Cond. Matt., 1:6291, 1989.

[80] U. Sivan, Y. Imry, and C. Hartzstein. Phys. Rev. B, 39:1242, 1989.

[81] G. Kirczenow. Phys. Rev. Lett., 62:2993, 1989.

[82] D. C. Ravenhall, H. W. Wyld, and R. L. Schult. Phys. Rev. Lett., 62:1780, 1989.

[83] G. Kirczenow. Solid State Commun., 71:469, 1989. 